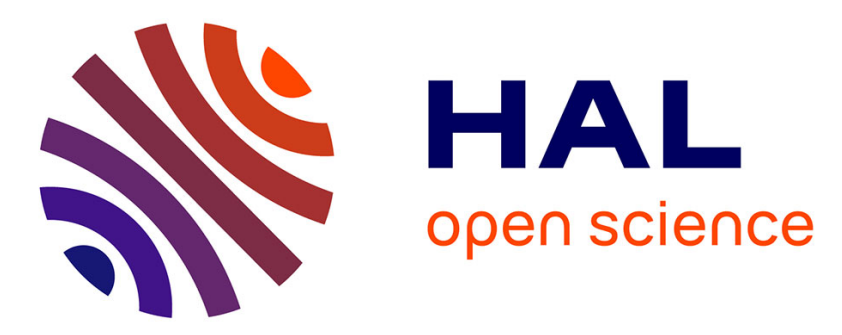

\title{
The colors of cometary nuclei - Comparison with other primitive bodies of the Solar system and implications for their origin
}

\author{
P. Lamy, I. Toth
}

\section{> To cite this version:}

P. Lamy, I. Toth. The colors of cometary nuclei - Comparison with other primitive bodies of the Solar system and implications for their origin. Icarus, 2009, 201 (2), pp.674. 10.1016/j.icarus.2009.01.030 . hal-00533506

\section{HAL Id: hal-00533506 https://hal.science/hal-00533506}

Submitted on 7 Nov 2010

HAL is a multi-disciplinary open access archive for the deposit and dissemination of scientific research documents, whether they are published or not. The documents may come from teaching and research institutions in France or abroad, or from public or private research centers.
L'archive ouverte pluridisciplinaire $\mathbf{H A L}$, est destinée au dépôt et à la diffusion de documents scientifiques de niveau recherche, publiés ou non, émanant des établissements d'enseignement et de recherche français ou étrangers, des laboratoires publics ou privés. 


\section{Accepted Manuscript}

The colors of cometary nuclei - Comparison with other primitive bodies of the Solar system and implications for their origin

P. Lamy, I. Toth

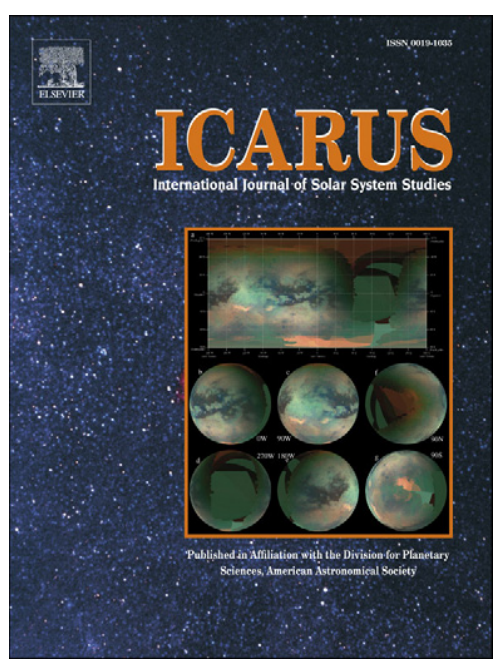

PII: $\quad$ S0019-1035(09)00065-7

DOI: $\quad$ 10.1016/j.icarus.2009.01.030

Reference: $\quad$ YICAR 8918

To appear in: Icarus

Received date: 18 January 2007

Revised date: 12 January 2009

Accepted date: 15 January 2009

Please cite this article as: P. Lamy, I. Toth, The colors of cometary nuclei - Comparison with other primitive bodies of the Solar system and implications for their origin, Icarus (2009), doi:

10.1016/j.icarus.2009.01.030

This is a PDF file of an unedited manuscript that has been accepted for publication. As a service to our customers we are providing this early version of the manuscript. The manuscript will undergo copyediting, typesetting, and review of the resulting proof before it is published in its final form. Please note that during the production process errors may be discovered which could affect the content, and all legal disclaimers that apply to the journal pertain. 


\title{
The colors of cometary nuclei - Comparison with other primitive bodies of the solar system and implications for their origin
}

\author{
P. Lamy \\ Laboratoire d'Astrophysique de Marseille, UMR6110 CNRS/Université de Provence, \\ Technopôle de Marseille-Etoile, 38 rue Frédéric Joliot-Curie, 13388 Marseille cedex 13, \\ France E-mail: philippe.lamy@oamp.fr \\ I. Toth \\ Laboratoire d'Astrophysique de Marseille, UMR6110 CNRS/Université de Provence, \\ Technopôle de Marseille-Etoile, 38 rue Frédéric Joliot-Curie, 13388 Marseille cedex 13, \\ France and Konkoly Observatory, Budapest H-1525, P.O. Box 67, Hungary
}

Received _ ; accepted _

Version 11 January 2009 
Running head: Colors of cometary nuclei

\section{Mailing address:}

\section{P. Lamy}

Laboratoire d'Astrophysique de Marseille, UMR6110 CNRS/Université de Provence, Technopôle de Marseille-Etoile, 38 rue Frédéric Joliot-Curie, 13388 Marseille cedex 13, France E-mail: philippe.lamy@oamp.fr 


\section{Abstract}

We present new color results of cometary nuclei obtained with the Hubble Space Telescope (HST) whose superior resolution enables us to accurately isolate the nucleus signals from the surrounding comae. By combining with scrutinized available data obtained with ground-based telescopes, we accumulated a sample of 51 cometary nuclei, 44 ecliptic comets (ECs) and 7 nearly-isotropic comets (NICs) using the nomenclature of Levison (1996). We analyze color distributions and color-color correlations as well as correlations with other physical parameters. We present our compilation of colors of 232 outer solar system objects - separately considering the different dynamical populations, classical KBOs in low and high-inclination orbits (respectively CKBO-LI and CKBO-HI), resonant KBOs (practically Plutinos), scattered-disk objects (SDOs) and Centaurs - of 12 candidate dead comets, and of 85 Trojans. We perform a systematic analysis of all color distributions, and conclude by synthesizing the implications of the dynamical evolution and of the colors for the origin of the minor bodies of the solar system. We find that the color distributions are remarkably consistent with the scenarios of the formation of TNOs by Gomes (2003) generalized by the "Nice" model (Levison et al., 2008), and of the Trojans by Morbidelli et al. (2005). The color distributions of the Centaurs are globally similar to those of the CKBO-HI, the Plutinos and the SDOs. However the potential bimodality of their distributions allows to possibly distinguish two groups based on their $(B-R)$ index: Centaur I with $(B-R)>1.7$ and Centaurs II with $(B-R)<1.4$. Centaurs I could be composed of TNOs (prominently CKBO-LI) and ultra red objects from a yet unstudied family. Centaurs II could consist in a population of evolved objects which have already visited the inner solar system, and which has been scattered back beyond Jupiter. The diversity of colors of the ECs, in particular the existence of very red objects, is consistent with an origin in the Kuiper belt. Candidate dead comets represent an ultimate state of evolution as they conspicuously appear more evolved than the Trojans and Centaurs II. 
Keywords: Comets, nucleus; comets, color; Kuiper belt Objects, color, Scattered Disk Objects, color; Centaurs, color; Jupiter Trojans, color; D-type asteroids, color;

\section{INTRODUCTION}

Primitive minor objects like Kuiper-belt objects (KBOs), Centaurs, cometary nuclei and low-albedo asteroids contain a considerable amount of information regarding the formation of early solar system planetesimals and some of the primordial accretion processes. Among the physical properties which can be investigated by remote observations, broadband colors remain one of the most powerful, especially for faint objects. Broadband colors by themselves offer limited insight into surface composition but correlations either between different color indices or with other (e.g., orbital) parameters can shed some light on the questions of the composition and the evolution of the minor objects. Furthermore, a systematic comparison of the color indices of various populations may provide clues on their relationships, and concur along with dynamical studies, to establish a scenario of their formation and evolution in the solar system.

The determination of the optical colors of these objects presents a formidable observational challenge as many of them are intrinsically faint. The bulk of cometary nuclei and several Centaurs present the further problem of being active and thus immersed in a coma. This explains the paucity of color data on cometary nuclei, long restricted to those which are inactive or do not sport a measurable coma. This latter case often requires observing the nuclei at large heliocentric distances where their intrinsic faintness further complicates the color measurements. Colors remain however for the time-being our best tool to characterize cometary nuclei as many near-infrared spectra obtained so far showed no sign of any absorption bands (e.g., Licandro et al. 2002, 2003; Abell et al. 2005; Campins et al. 2006). There are two exceptions coming from in-situ space observations, the $2.19 \mu \mathrm{m}$ 
band discovered on the nucleus of 19P/Borrelly (Soderblom et al. 2004) whose origin is currently unknown (an instrumental artifact cannot be excluded) and the water-ice bands at 1.5 and $2.0 \mu \mathrm{m}$ identified on the nucleus of 9P/Tempel 1 (Sunshine etal. 2006).

In an effort to circumvent the paucity of cometary data, Jewitt and Luu (1990) characterized the colors of 32 jovian Trojans as "spectral analogs to cometary nuclei". They found that the reflectivity gradients of their cometary sample - two nuclei, 2P/Encke and 10/Tempel 2 - fall within the range defined by the Trojans. Luu (1993) and Luu and Jewitt (1996) broadened the comparison by including Centaurs and KBOs but with only 4 cometary nuclei, 1 Centaur and 5 "cometary nucleus candidates" for the former, and 11 KBOs, 4 Centaurs and 6 nuclei for the latter in their samples, their conclusions cannot be considered very robust. In their large compilation, Hainaut and Delsanti (2002) had 12 cometary nuclei and much larger numbers of KBOs and Centaurs. They focused on KBOs, looking for correlations with other physical properties but noted that cometary nuclei are significantly bluer than the other minor bodies in the outer solar system. Jewitt (2002a,b) presented the first real attack on the question of the contribution of colors to the relationship between supposedly related populations of minor objects. His analysis included 28 KBOs, 9 Centaurs, 12 cometary nuclei, 12 candidate dead comets and 32 Trojans. He concluded that the color distributions of cometary dead comets and cometary nuclei are indistinguishable and that they are both lacking the ultra-red colors seen in the KBOs and in Centaurs. The Meudon Multicolor Survey has led to several correlation studies, the most recent and complete one being that of Doressoundiram et al. (2005). Their data set now culminates to $58 \mathrm{KBOs}$ and 9 Centaurs, and their analysis further included 8 cometary nuclei from our compilation (Lamy et al., 2004) and 19 irregular satellites. They found that the only statistically significant correlation of cometary colors was with those of SDOs but warned that this conclusion cannot be taken as definitive owing to the small number of objects. The question is crucial because the dynamical analysis, while all converging 
towards an origin of ecliptic comets in the Kuiper belt, are presently unable to decide on the relative contributions of the different dynamical groups and incidentally, on the dominating delivery mechanism, whether dynamical or collisional (Farinella et al., 2000). Snodgrass et al. (2006) compared the colors of 14 cometary nuclei with those reported by Jewitt and Luu (2001) and Peixinho et al. (2004) on 62 KBOs and 9 Centaurs, but limited their analysis to a single color-color correlation, namely $(R-I)$ versus $(V-R)$. They concluded that while the nuclei and KBOs follow the same trend in color contrary to Centaurs, the averages $(V-R)$ of the former two populations differ by 0.13 . Fornasier et al. (2007) performed a systematic comparison of the colors of the Jupiter Trojans (80 objects) with those of other populations of minor bodies: 96 classical KBOs, 34 SDOs, 38 Plutinos, 30 Centaurs, and 19 cometary nuclei. They concluded that the mean colors of the Trojans, excluding the Eurybates family, are similar to those of the nucleus of ecliptic comets and neutral Centaurs, but that their color distributions are different. Tegler et al. (2008) presented the histograms of the $(B-R)$ color index of eight different groups of outer system solar objects (Centaurs, comet nuclei, Jovian and Neptunian Trojans, Plutinos, cold classical KBOs, SDOs, and irregular satellites of Jupiter, Saturn, and Neptune) but their discussion concentrated mostly on explaining possible processes responsible for the color bimodality of Centaurs.

The purpose of the present article is to reconsider the above questions on the basis of much larger data sets. We first present new color results on cometary nuclei obtained with the Hubble Space Telescope (HST) whose superior resolution enables us to accurately isolate the nucleus signals from the surrounding comae. By combining with scrutinized available data mostly obtained with ground-based telescopes, we accumulated a sample of 51 cometary nuclei, 44 ecliptic comets (ECs) and 7 nearly-isotropic comets (NICs) using the nomenclature of Levison (1996). We analyze color distributions and color-color correlations as well as correlations with other physical parameters. We then present our 
own compilation of colors of 282 objects in the outer solar system, separately considering the different dynamical populations, classical KBOs in low and high-inclination orbits, resonant KBOs (practically Plutinos), scattered-disk objects (SDOs) and Centaurs. We perform systematic comparisons of color distributions, and conclude by synthesizing the implications of the colors for the origin of the different populations.

\section{THE COLORS OF COMETARY NUCLEI}

\subsection{Hubble Space Telescope observations}

All observations were performed with the Planetary Camera (PC) mode of the WFPC2 of the Hubble Space Telescope which offers an image scale on the detector of $0.045 \mathrm{arcsec} / \mathrm{pixel}$. The observations were organized so as to optimize the use of the total exposure time available during an HST orbit ( 30 minutes including overhead). The scheme entirely depends upon the expected brightness of each nucleus and is a case by case decision. For the comets of interest in this present study, two pairs of identical exposures were always taken with two broadband filters, one pair with the F555W and one pair with the F675W. In the case of short exposure times, observations with the F814W and F439W filters were obtained, and with the F336W filter in only one case, comet 45P/Honda-Mrkos-Pajdusakova. Table 1 gives the characteristics of these filters (Biretta et al. 1996). Image pairs are generally secured to detect artifacts coming, for instance, from cosmic ray impacts or from imperfect tracking (very rare). Table 2 presents the journal of the observations of the twenty four nuclei for which we could obtain color information. Thirteen were observed during a single orbit while the other nuclei were observed on several orbits, from two to twelve. A full color sequence is performed during each orbit and extends over less than thirty minutes thus minimizing possible color variations associated with the rotation of the nuclei. All images were processed using the standard "On The Fly" (OTF) 
pipeline calibration system at the Space Telescope Science Institute, which includes bias subtraction and flat-fielding corrections. A full discussion of all the steps in the pipeline reduction process can be found in Holtzman et al. (1995).

To analyze the data, we applied our standard method of fitting a parametric model of the expected surface brightness of the comet composed of a nucleus and a coma to the observed images (e.g., Lamy et al. 1998a, 1998b, 1999, 2002). The brightness distribution for the comet is modeled as

$$
B(\rho)=\left[k_{c} / \rho^{p}+k_{n} \delta(\rho)\right] \otimes P S F
$$

where $\rho$ is the radial distance from the nucleus, $\delta(\rho)$ is the Dirac delta function, and $\otimes$ is the convolution operator. The first term represents the coma with the scaling factor $k_{c}$ while the second term is the contribution of the nucleus with the scaling factor $k_{n}$. The point spread function of the telescope was modeled using version $4.0 \mathrm{~b}$ of the TinyTIM software written by Krist (1995) which has the capability of including the effect of the jitter of the HST, sometime conspicuous on long exposures. In order to take into account the sub-pixel location of the nucleus, which may cause a marked asymmetry of the brightness profiles, the model images were generated on a finer grid than the original PC pixel, with a resampling factor of 9. The fit to the real images was performed after integrating the model over $9 \times 9$ sub-pixels to recover the original pixel of the PC. The scaling factors $k_{n}$ and $k_{c}$, the power exponents $p$, the sub-pixel locations of the nucleus $\left(x_{n}, y_{n}\right)$ and the appropriate jitter if any were determined individually on each image by minimizing the residuals between the synthetic and the observed images. The fits were performed on the $X$ and $Y$-profiles for 45P/HMP (Lamy et al. 1999). For all other nuclei, they were performed on the $X$ and $Y$-profiles to determine $\left(x_{n}, y_{n}\right)$, and on the azimuthally averaged radial profiles to determine the other parameters. Figures 1a to 1e display one HST image and a typical fit 
of the radial profiles for the 18 comets not yet published. In all cases, the inner coma does not significantly departs from axisymmetry justifying the use of the simple isotropic model (Eq. 1).

The determination of the absolute magnitudes was performed on the $k_{n} P S F$ images, which measure the brightness of the nucleus as it would be observed by the HST in the absence of coma. The procedure followed the recommendations of Holtzman et al. (1995). The so-called instrumental magnitudes were calculated by integrating the scaled PSFs in an aperture of 0.5 arcsec radius, so that no aperture correction is required. The formulae converting the instrumental WFPC2 magnitudes to the standard Johnson-Kron-Cousins $B V R I$ magnitudes require color corrections in first and second orders, i.e., involve terms like $(V-R),(V-I),(B-V)$, and their squares. The formulae were combined two at a time to generate and solve second-order equations for the color terms. This procedure allows to solve the system of 2 or 3 equations (depending upon the case) in a self-consistent way and to retrieve the standard magnitudes without any assumption on the color of the nuclei. For the purpose of the present study, the individual magnitudes were averaged to produce robust determinations of the colors. For those comets observed over several orbits (eight orbits typically extend over a time span of $11.5 \mathrm{hr}$ ), this procedure smoothes any color variations associated with inhomogeneous nuclear surfaces.

The uncertainties in the magnitudes result from the noise in the data and from the fitting procedure while errors introduced by the transformation formulae are negligible. The noise from counting statistics is discussed in our previous articles and is further efficiently reduced by averaging 360 azimuthal profiles. The dominant source of uncertainty lies therefore in the fitting procedure. The nuclei of all comets presented here were easily detected with a good contrast with respect to their coma (the signals of the nuclei always exceeded those of the comae in the central pixels). The uncertainties affecting the $k_{n}$ 
scaling factors were estimated by a global quantification of the final residuals between the synthetic and observed images. These uncertainties were propagated through the various transformation formulae to yield the uncertainties on the color indices.

Our results are summarized in Table 3. Detailed analysis of several nuclei have already been published: 22P/Kopff (Lamy et al. 2002), 45P/HMP (Lamy et al. 1999), 46P/Wirtanen (Lamy et al. 1998a), 67P/Churyumov-Gerasimenko (Lamy et al. 2006). The others will be the subject of forthcoming articles: Lamy et al. (2009) and Lamy et al. (in preparation).

\subsection{Other observations of the colors of cometary nuclei}

Table 4, adapted from Table 5 of Lamy et al. (2004), summarizes all presently available data on the colors of cometary nuclei. The $(V-R)$ values for $26 \mathrm{P} /$ Grigg-Skjellerup and 73P/Schwassmann-Wachmann 3 were obtained by Boehnhardt et al. (1999) using Johnson $V$ and $R$ filters. We transformed them to the standard Johnson-Kron-Cousins system using Eq. 3 introduced below, and further assuming constant reflectivity gradients in the range 550-700 $\mathrm{nm}$. We checked that the transformation formula given by Fernie (1983) yields similar results, within $0.02 \mathrm{mag}$. The color indices of the nucleus of $162 \mathrm{P} /$ Siding Spring were calculated from the constant reflectivity gradient $S_{\lambda}^{\prime}=9.2 \pm 1.0 \% / 100 \mathrm{~nm}$ determined by Campins et al. (2006) and valid from 360 to $900 \mathrm{~nm}$. Data on the nuclei of 22P/Kopff, 46P/Wirtanen, 87P/Bus and 152P/Helin-Lawrence reported by Meech et al. (2004) were excluded because they were still active although observed at large heliocentric distances. The recent data of Snodgrass et al. (2008) were carefully scrutinized leading to the following choices.

- All comets which displayed some form of activity, coma or tail, were excluded with 
the exception of 94P/Russell 4 (in spite of some possible faint contamination by a coma or bright nearby stars, its colors are typical of ecliptic comets).

- 44P/Reinmuth was also excluded as it was very faint and observed under poor seeing conditions [we further note that their $(V-R)=0.31 \pm 0.09$ is inconsistent with our $\operatorname{HST}$ result $(V-R)=0.62 \pm 0.08]$.

- For 36P/Whipple, we favored their extended color coverage performed in March 2006 $[(V-R)=0.48 \pm 0.03$ and $(R-I)=0.62 \pm 0.02)]$ against other results of inferior quality.

- For 121P/Shoemaker-Holt 2, we kept only the data obtained in March 2006 as the $(V-R)$ index measured in May 2006 has a very large uncertainty of 0.2 .

The inactive or weakly active nuclei 2P/Encke, 10P/Tempel 2, 28P/Neujmin 1, 49P/ArendRigaux, and 143P/Kowal-Mrkos are of no particular concern and all measurements are in good agreement taking into account the respective uncertainties. For two nuclei 19P and $48 \mathrm{P}$, the uncertainties are quite large, respectively \pm 0.78 and \pm 0.30 , making their $(V-R)$ index of limited value.

Table 5 presents the adopted color indices calculated as the weighted average of the individual colors where the weight of a measurement is set to $1 / \sigma$. The final error is calculated as a combination of the individual errors and of the dispersions of the measurements around their mean according to Eq. 2 of Hainaut and Delsanti (2002). Five nuclei listed in Table 5 were observed with the HST. For 10P/Tempel 2, the HST determination $(V-R)=0.49 \pm 0.03$ and the mean of the ground-based values $(V-R)=0.56 \pm 0.03$ agree within their error bars and the weighted average of all (HST + ground-based) measurements yields our adopted value $(V-R)=0.54 \pm 0.03$. For $17 \mathrm{P} /$ Holmes, the two determinations agree at the $2 \sigma$ level. For $47 \mathrm{P} /$ Ashbrook-Jackson, 
the agreement of the three determinations is even better, almost at the $1 \sigma$ level. For $67 \mathrm{P} /$ Churyumov-Gerasimenko, the HST determination $(V-R)=0.51 \pm 0.06$ and that of Tubiana et al. (2008) $(V-R)=0.54 \pm 0.03$ are in excellent agreement. For 86P/Wild 3, the difference between the two determinations of $(V-R)$ amounts to 0.74 mag! It turns out that this nucleus was observed from the ground while very faint and very close to a bright star (Hainaut, private communication), thus invalidating the puzzling result of a very blue color.

With the exception of $86 \mathrm{P} /$ Wild 3 , the ground-based results were merged with our HST results bringing the sample to a total of 51 nuclei. There are 44 ecliptic comets (ECs) and 7 nearly-isotropic comets (NICs), using the classification scheme proposed by Levison (1996) based on the Tisserand parameters of the comets with respect to Jupiter.

\subsection{The reflectivity spectra of cometary nuclei}

Figure 1 displays the reflectivity spectrum $S(\lambda)$ of the nuclei calculated from the observed color indices minus the solar color indices and normalized to a value of 1 at $550 \mathrm{~nm}$, the effective wavelength of the $V$ band; they measure the color of the nuclei relative to that of the Sun. The normalized reflectivity gradient $S_{\lambda}^{\prime}$ in a given spectral domain $\lambda_{1} \leq \lambda \leq \lambda_{2}$ was introduced by Luu and Jewitt (1990a) to quantify the spectra of a limited number of objects which could reasonably be fitted with a straight-line from $\sim 400$ to $\sim 850 \mathrm{~nm}$ :

$$
S_{\lambda}^{\prime}\left(\lambda_{1}, \lambda_{2}\right)=(d S / d \lambda) / S_{\lambda}
$$

Figure 2 reveals such a diversity of spectral behaviors that this approximation is no longer appropriate and the gradient $S_{\lambda}^{\prime}$ looses its interest. In fact, fitting a straight-line would hide the complexity of several spectra. One could define a gradient in a restricted spectral 
range, for instance $\Delta \lambda=[550,650 \mathrm{~nm}]$ but then $S^{\prime}$ becomes strictly equivalent to the color index $(V-R)$ since

$$
(V-R)=(V-R)_{\odot}+2.5 \log \left[\frac{2+S^{\prime} \Delta \lambda}{2-S^{\prime} \Delta \lambda}\right]
$$

Consequently we will discuss our results in terms of the color indices which are further readily available for related minor objects in the solar (KBOs, Centaurs, Trojans).

\subsection{The histograms of the colors of cometary nuclei}

Figure 3 displays the histograms of the four color indices $(B-V),(V-R),(R-I)$ and $(B-R)$ constructed with a bin size of 0.1 . The gaps must not be overinterpreted as suggesting different groups but most likely result from the (still) limited coverage, particularly for the $(B-V)$ and $(B-R)$ colors. Later on, we shall introduce the cumulative distribution function (CDF) which is less intuitive than histograms but not prone to artifacts. The $(V-R)$ color index for 44 ECs ranges from slightly blue to ultra-red (0.86), when compared to the color of the Sun $\left[(V-R)_{\odot}=0.35\right]$; its mean value amounts to 0.49 with a standard deviation of 0.094 . We point out that the very large uncertainties affecting the $(V-R)$ index of the two bluest nuclei, 14P/Wolf $((V-R)=0.02 \pm 0.22)$ and 19P/Borrelly $((V-R)=0.25 \pm 0.78)$, so that they could well be in reality less blue than implied by Fig. 3. The $(B-V)$ index for 15 ECs extend from 0.72 to 1.58 , a range of color systematically redder than the Sun for which $(B-V)_{\odot}=0.65$. The $(R-I)$ index for 25 nuclei ranges from 0.21 to 0.66 but only two nuclei (45P/Honda-Mrkos-Pajdusakova and $59 \mathrm{P} /$ Kearns-Kwee) are slightly bluer than the Sun which has $(R-I)_{\odot}=0.28$. The $(B-R)$

index for 15 ECs ranges from 0.8 to 1.7 but only one nucleus (107P/Wilson-Harrington) is slightly bluer than the Sun which has $(R-I)_{\odot}=1.0$. The meager color data we presently 
have on the nuclei of NICs indicate less diversity of colors and the absence of blue and very red nuclei. It will be interesting to see whether those trends are confirmed when the sample of NICs will expand. The spectral diversity among the nuclei of ECs was perceived by Luu (1993) based on only four of them but the extremely large range of colors that our observations uncovered is totally unexpected and quite surprising.

\subsection{The color-color relationship for cometary nuclei}

As already pointed out by Luu and Jewitt (1996), broadband colors by themselves offer limited insight into surface composition, but a systematic correlation between different color indices could suggest trends in surface composition. Figure 4 displays five different color-color diagrams corresponding to the various combinations of indices. Following Hainaut and Delsanti (2002), we plotted "reddening lines" constructed by calculating the colors of an object having a given normalized reflectivity gradient $S^{\prime}$ using Eq. 3 and connecting all the points in the range $-10<S^{\prime}<+50 \% / 100 \mathrm{~nm}$. An object located on such a line has therefore a linear normalized reflectivity spectrum in the spectral range defined by the color index, and its gradient can be roughly estimated using the tick-marks on the line. The interest of the "reddening lines" stems from the fact that the three physical processes - aging, collision and activity - that are suspected to control the colors of the minor bodies in the solar system independently produce linear reflectivity spectra over the visible wavelength range, at least in a first approximation. Any combination of the above processes should also result in the same property. In this framework and further assuming that the objects have all the same composition, their colors should all lie on the "reddening lines". Figure 4 shows that the colors of some of the cometary nuclei tend to be correlated and to cluster close to the "reddening lines". There are also several "outliers" as their colors and respective uncertainties clearly exclude them from the above trend: $37 \mathrm{P}, 45 \mathrm{P}, 50 \mathrm{P}, 59 \mathrm{P}$, 
$86 \mathrm{P}$ and 106P. The color data come from our HST observations ensuring that they were not contaminated by comae. The case of $45 \mathrm{P}$ is worth pointing out because its coma was extremely faint and had a very different color compared to that of its nucleus (see Fig. 11 and 12 in Lamy et al. 1999).

To estimate the significance of the color-color relationship, we computed the Spearman rank correlation $\rho$ together with the associated significance level (SL) as recommended by Peixinho et al. (2004), and also introduced by Trujillo and Brown (2002) in their analysis of the correlation between inclination and color in the classical Kuiper Belt. The strength of the Spearman method is that it is distribution free, i.e., it does not presuppose a functional form for the correlation as do linear least-squares estimates. In addition, the Spearman method is less sensitive to the presence of deviant data points than a $\chi^{2}$ correlation test, as it is the difference in rank between sorted data lists that is compared, not the actual data values themselves. On the flip side, this method does not take into account the uncertainties in the data. If some error bars are larger than the differences between some data points and/or if the datasets are small, the ranking will be affected just by moving the corresponding data points along their errors bars. Likewise Trujillo and Brown (2002), we introduced two statistical tests, the t-statistic and the variance of the sum squared difference of ranks, $\operatorname{Var}(D)$, to estimate the significance of a nonzero value of $\rho$; they always yielded similar results within a few percents. Application of this test to our global set of colors shows that there is no statistically significant correlations.

\subsection{The color-distance relationship for cometary nuclei}

Color variations as a function of heliocentric distance have been reported within the asteroid belt (Gradie and Tedesco, 1982; Gradie et al., 1989) and possibly within the classical Kuiper belt (see below). Figure 5 shows the color index of the cometary nuclei 
plotted versus their perihelion distances. The variations of the $(V-R)$ index suggest that there could be two distinct groups, the red nuclei whose reddening tends to increase with increasing perihelion distance and the blue nuclei whose $(V-R)$ color tends to be independent. We calculated the Pearson linear correlation coefficient $C_{P}$ for the group of the red nuclei, i.e., excluding the blue ones. With $C_{P}(V-R)=0.69 \pm 0.13$, the $(V-R)$ indices of the red nuclei do appear to vary quasi linearly with the perihelion distance. However such a correlation is not convincing for the two other indices as we obtained $C_{P}(B-V)=0.34 \pm 0.30$ and $C_{P}(R-I)=0.43 \pm 0.33 . C_{P}(B-R)=0.28 \pm 0.32$. Turning our attention to the blue nuclei, it appears that their color indices are quasi independent of perihelion distance. Application of the Spearman test described in the above section shows that the significance levels of the color-distance relationship never exceed $68 \%$, too low to ascertain a correlation.

\subsection{The color-inclination and color-dynamical lifetime relationships for cometary nuclei}

The question of a possible correlation between color and the age of cometary nuclei can be investigated by considering either the orbital inclination $i$ or the parameter $d_{J}$ (defined in Levison and Duncan, 1997), both being a proxy to the age of comets. The median values of $\mathrm{i}$ and $\mathrm{d}_{J}$ increase with time and the numerical simulations of Levison and Duncan (1997) confirmed that the inclination distribution of ECs, when they first become observable, is more concentrated to small inclinations than the observed population, and that it becomes more distended as it aged. Precisely, this means that all the objects with large $i$ and $d_{J}$ are old, and that all objects with small $i$ and $d_{J}$ are a mixture of old and young objects. As color is also supposed to change with aging as well, color-inclination and color-dynamical lifetime relationships are plausible. We note that the former relationship 
has been suspected within the classical Kuiper belt (see below subsection 3.1.1). Figure 6 shows the color indices of the cometary nuclei plotted versus their inclination together with the Pearson linear regressions. With the exception of the $(R-I)$ index, all other indices and particularly those involving the $B$ band exhibit a decreasing trend with increasing inclination, consistent with the aging effect mentioned above. The Spearman test for these two indices yielded significance levels of $89 \%$, however generally considered not large enough to disprove the absence of correlation. Figure 7 shows the color indices of the cometary nuclei plotted versus this parameter together with the Pearson linear regressions. On the one hand, all indices with the exception of $(B-V)$ display the expected decreasing trend with increasing dynamical lifetime. On the other hand, the situation is in partial contradiction with the color-inclination relationship. In particular the two indices which involves the $B$ band exhibit opposite behaviors which was not the case for the latter relationship. However this result may be pondered as the Spearman test yields very low values of $\rho$ with significance levels of respectively $98 \%$ for $(B-V)$ and $93 \%$ for $(B-R)$. It is therefore difficult to conclude about the reality of a color-dynamical lifetime correlation on the basis of the present data set.

\subsection{The color-size relationship for cometary nuclei}

Figure 8 displays the four color indices of the cometary nuclei as functions of their effective radii tabulated in Lamy et al. (2004) and in Snodgrass et al. (2006, 2008) for more recent data. There is an apparent trend for the range of colors to decrease as the size increases, the sub-kilometer nuclei exhibiting the broadest range, from slightly blue to ultra-red. Table 6 presents a quantitative assessment of this trend and displays the means and variances of the distributions of the three color indices separately for the sub-kilometer and kilometer size nuclei with a cut-off at a radius of $2 \mathrm{~km}$. Two determinations are 
presented, unweighted and weighted according to the method described in subsection 2.2. For all three color indices, the differences between the two size ranges never exceed the uncertainties. The Spearman test confirms that there are no statistically significant correlations of color with nucleus size.

\subsection{The color-active fraction relationship for cometary nuclei}

Following the in-situ observations of the nucleus of 1P/Halley in 1986, the idea prevailed that all cometary nuclei were alike, with most of the surface covered by an inactive mantle, the activity being limited to a few vents whose cumulated area is no more than a few percents of the total area of the nucleus (the ratio of the two areas defines the active fraction). This idea was further reinforced by the in-situ observation of 19P/Borrelly in 2001, which shows the same properties. As new size determinations are available as well as extended measurements of water production rates (e.g., A'Hearn et al. 1995), it now appears that cometary nuclei, in particular the sub-kilometer ones, may have very large active fractions, in fact as much as $100 \%$ of their surface such as $46 \mathrm{P} /$ Wirtanen (Groussin

and Lamy, 2003). The reality is certainly a spectrum of very different situations, from nuclei still having preserved much of their original (irradiation ?) mantles (e.g., Hale-Bopp) to bare nuclei resulting from widespread activity to nearly extinct covered with a rubble crust. In fact, the reality is more complex as the surface of a given nucleus may be in different states depending upon its location along its orbit. The active fraction of many nuclei is obviously a function of heliocentric distance so that a single value cannot be defined. Moreover, a rubble crust may form post-perihelion whose properties will be radically different from the underlying fresh surface. According to the scenario of the evolution of the surface of 46P/Wirtanen by Groussin and Lamy (2003), such a rubble crust was likely present at the time of our HST observations (Lamy et al., 1998a). Despite these difficulties, 
we attempted to investigate a possible trend of color with the pattern of activity of the nucleus surfaces. For those nuclei common to our color data base and A'Hearn et al. 1995's data base, we calculated the active fractions as the ratios of their tabulated active areas (areas of free-sublimating ice required by the measured $\mathrm{OH}$ production rates) to the surface areas of spherical nuclei using the effective radii tabulated by Lamy et al. (2004). Figure 9 displays the result for the $(V-R)$ index for fifteen ECs and three NICs. No clear pattern emerges and in fact, i) nuclei having approximately the same active fraction may have very different colors and ii) nuclei having nearly same color may have widely different active fractions (e.g., $21 \mathrm{P}$ and $28 \mathrm{P}$ ). It is very likely that the situations are too diverse and too complex as outlined above to be reflected by such a simple diagram.

\section{COMPARISON WITH OTHER MINOR OBJECTS IN THE SOLAR SYSTEM}

The purpose of this section is to perform a general comparison of the colors of cometary nuclei with those of other minor objects in the solar system. We are particularly interested in the relationship between the color (as a proxy to physical properties) of cometary nuclei with those of their supposed progenitors. Testing genetic relationships has thus far focused on dynamical studies because so little is now about their physical properties. Growing efforts are ongoing to explore these properties to provide independent clues on the origin and evolution of these objects. Our much larger data set compared to that used by Jewitt (2002a), Doressoundiram et al. (2005) and Fornasier et al. (2007) allows us to look at the problem in much more detail. Our endeavor first requires that we briefly describe the different families of minor objects in the solar system, summarize their main properties, and review the current scenarios of their origin and their interrelations resulting from the recent explosion of dynamical studies. Oort cloud objects are left out of this study as we 
simply do not have direct observations although some of the NICs may offer a first hint of their properties. We present the sources of color data and summarize the main trends but we do not address question specific to the colors of the minor objects as this is well dealt in various articles.

\subsection{Trans-Neptunian Objects (TNOs)}

In the views of the individuals whose initially speculated about the existence of objects beyond the orbit of Neptune (Leonard, Edgeworth, Kuiper, see Luu and Jewitt, 2002), they would form a belt (named the "Kuiper belt" by Duncan et al., 1988) with low-inclination, nearly circular orbits. It is now realized that the Kuiper belt, which is considered to have its inner edge defined by the orbit of Neptune $\left(a_{N}=30 \mathrm{AU}\right)$ has been extensively sculpted by the gravitational influence of the giant planets and particularly Neptune itself. ¿From the orbits of known TNOs, three main distinct dynamical groups have emerged, the classical KBOs, the resonant KBOs and the SDOs, that is the scattered-disk objects (see the reviews by Jewitt, 1999; Morbidelli, 1998; Jewitt and Luu, 2000; Malhotra et al, 2000; Gladman et al., 2001, 2002; Luu and Jewitt, 2002; Schulz, 2002; Morbidelli et al., 2003; Morbidelli and Brown, 2004; Chiang et al., 2007; Gladman et al., 2008; Morbidelli et al., 2008).

\subsubsection{Classical KBOs - The classical Kuiper Belt}

Presently the majority of the known KBOs form the classical Kuiper belt and are according called classical KBOs, in short CKBOs. They lie outside resonances and are characterized by semi-major axes $42 \leq a \leq 47 \pm 1 \mathrm{AU}$ and perihelion distances $q>35 \mathrm{AU}$. There seems to be a consensus that the classical Kuiper belt has a sharp outer boundary at $\sim 48 \mathrm{AU}$, the present 1:2 mean motion resonance with Neptune. Contrary to first 
expectations, the distributions of inclination and eccentricity are unexpectedly broad. It is now accepted that two distinct populations co-exist in the classical Kuiper belt based on their different inclination distributions (Brown, 2001; Levison and Stern, 2001; Trujillo and Brown, 2002). The reality of these two populations is reinforced by different color properties and possibly different sizes. The population of CKBOs with $i \lesssim 4^{\circ}$, the "cold" population, was long thought to be primordial since it is dynamically stable over the age of the solar system (Duncan et al., 1995; Kuchner et al., 2002). They were considered to have formed at or very close to their current locations and to have evolved by resonance sweeping (Malhotra, 1993); they would therefore have very similar physical characteristics because they were formed at the same time and within a narrow region. It was further thought that some mechanism excited the classical (low-inclination, low-eccentricity) belt during the early stage of its formation resulting in the creation of the population of CKBOs with $i \gtrsim 4^{\circ}$, the "hot" population. Difficulties have soon appear with this simple scenario, in particular the huge mass deficit $(\sim 99.9 \%)$ of the present population with respect to what models predict for the KBOs to accrete in situ. The idea that the "hot" population did not form in-situ but closer to the Sun, and was subsequently transported outward during the final stage of planets formation was first proposed by Levison and Stern (2001), and later elaborated by Gomes (2003) on the basis of the migration of Neptune. This idea was then generalized to the "cold" population by Levison and Morbidelli (2003), and they proposed that the entire population of Kuiper belt objects formed within a primordial edge at 30-35 AU and was transported outwards to its current location during Neptune's migration (see also Gomes et al., 2004). As recognized by Morbidelli et al. (2008), this model had several difficulties, in particular in explaining the different physical properties of the cold and hot populations, because both would have originated within $35 \mathrm{AU}$, although in somewhat different parts of the disk.

In its most recent and elaborated version, a chaotic phase in the migration of Neptune 
(and Uranus as well) is introduced as resulting from the dynamical evolution of the four ice giants assumed to be on nearly-circular and coplanar orbits much closer than they presently are. The growing eccentricity of Uranus and Neptune allows them to deeply penetrate into the planetesimal disk and to scatter most of it all over the solar system. When their eccentricity damps out, the Kuiper belt becomes stable, and the objects that happen to be there at that time remain trapped thus forming the cold population, the process being fast enough to prevent any excitation. The hot population is then captured later, when Neptune is migrating up to its final orbit on a low-eccentricity orbit, as proposed by Gomes (2003). This scenario, known as the "Nice model" (Levison et al., 2008) is very appealing since it successfully explains many features of the solar system, including the capture of the Trojans (see below).

The color data of the KBOs used in this present study result from our own compilation of many sources: Luu (1993), Luu and Jewitt (1996), Green et al. (1997), Barucci et al. (1999, 2000), Boehnhardt et al. (2001, 2002), Doressoundiram et al. (2001, 2002, 2005), Hainaut and Delsanti (2002), Jewitt and Luu (1998, 2001), Mueller et al. (2003), Peixinho et al. (2003, 2004), Stephens et al. (2003), Tegler and Romanishin (1998, 2000, 2003), Gulbis et al. (2006), and DeMeo et al. (2008). We collected color indices of 111 CKBOs as detailed in Table 7 . When multiple results were available, we calculated the weighted color indices and the associated uncertainties as described in subsection 2.2. This method gives more weight to the most accurate measurements. Typical errors range from 0.05 to 0.20 mag.

According to the analysis of Doressoundiram et al. (2002, 2005), Peixinho et al. (2004) and Trujillo and Brown (2003), classical KBOs appear to be composed of two different populations in agreement with the orbital analysis: objects with $i<4.5^{\circ}$ have only red colors while those with $i>4.5^{\circ}$ exhibit a broad range of colors, from blue to very red. 
This is also consistent with the finding of Gulbis et al. (2006) that the colors of KBOs in the Core (low-inclination) and Halo (high-inclination) are statistically different. The Core-Halo classification scheme introduced by Elliot et al. (2005) based on the inclination with respect to the mean orbital plane of the KBOs, is sufficiently similar - although not strictly identical - to the dynamically "hot" and "cold" populations that the distinction is unimportant for our present purpose. These two populations further show different color-color correlations suggesting intrinsic differences. The brightest objects $\left(H_{R}<6.2\right)$ have their color highly correlated with perihelion distance $[(B-R)$ increases with $q]$, but this trend may be contaminated by the color-inclination correlation or vice-versa. This results from the fact that objects with $H_{R}>6.2$ exhibit a strong correlation between their perihelion distances and their inclinations. Doressoundiram et al. (2005) have however noted that the correlations of colors with inclination and perihelion distance are no longer present when a dynamically more restricted definition is adopted for the classical KBOs. Peixinho et al. (2008) have presented a slightly different interpretation: objects with $i<12^{\circ}$ are homogeneously red while objects with $i>12^{\circ}$ show bluish colors which are either weakly correlated with inclination or are simply homogeneously blue; however inspection of their Fig. 1 and 2 reveals that their color break at $i=12^{\circ}$ is not really superior to that at $i \simeq 5^{\circ}$. Peixinho (2008) has pointed out that the color correlations with inclination and perihelion distance are probably an artifact of a more relevant relationship between colors and the Tisserand parameter measured with respect to Neptune $T_{N}$ as already noted by Chiang et al. (2007) and Jewitt et al. (2007). Accordingly CKBOs with $T_{N}>3.05$ are homogeneously ultra-red whereas those with $T_{N}<3.05$ have rather mixed colors from slightly blue to moderately red. Whatever the final word, the co-existence of two distinct populations is further reinforced by two lines of evidence.

- CKBOs with $i<5.5^{\circ}$ exhibit a much larger fraction of binaries than those with larger inclinations (Noll et al. 2008); the same trend holds true for the number of 
similar-brightness system.

- Cold CKBOs have distinctly higher visual albedos than the hot ones and other TNO dynamical classes (Grundy et al. 2005, Stansberry et al. 2008, Brucker et al. 2009, Grundy et al. 2009).

As already pointed out, the "Nice model" offers a simple, natural explanation for the observed differences in the physical properties since the two populations originate from different parts in the planetesimal disk: the cold population comes, almost exclusively, from the outermost parts of the planetesimal disk beyond 29 AU while the hot population comes from the inner disk.

A further subdivision of CKBOs has recently appeared: the inner Classical KBOs which have mean semi-major axes less than $39.46 \mathrm{AU}$ (interior to the 3:2 resonance with Neptune, the current position of the plutinos). They may represent the surviving members of the primordial Kuiper Belt that formed within this distance. The $(B-R)$ index of 10 of those objects reveal a mixture of neutral and red colors with a distribution comparable to those of plutinos or scattered disk objects but significantly different from that of the CKBOs (Romanishin et al. 2008). Further measurements are necessary to ascertain this new intriguing finding.

\subsubsection{Resonant KBOs - The Plutinos}

The resonant KBOs occupy mean resonances with Neptune. The 3:2 resonance at $a=39.46 \mathrm{AU}$ is the most densely populated and includes Pluto, hence the classification of these trapped KBOs as Plutinos. Some of them, like Pluto itself, have perihelia $q \leq 30 \mathrm{AU}$ and their orbits cross that of Neptune. They are however protected from destabilizing close encounters by their location in the resonance. Other populated resonances include the 2:1 
at $a=47.8 \mathrm{AU}$ and the $4: 3$ at $a=36.4 \mathrm{AU}$. A process of populating Neptune's resonances has been proposed by Malhotra $(1995,1998)$ and Malhotra et al. (2000), and is based on the radial migration of the giant planets as conjectured by Fernández and Ip (1984). As Neptune moved outwards, its mean motion resonances swept ahead of it through the planetesimal disk and captured Pluto and many Plutinos into the 3:2 resonance and other KBOs in other resonances but with lower efficiency. The dynamics of resonances implies rather large eccentricities and inclinations, but some orbits have such large values that an external excitation may be required. This difficulty is alleviated by the work of Gomes (2003) who proposed that the resonant KBOs have the same origin as the high-inclination CKBOs.

The color data used in the present study come from on own compilation as described in subsection 3.1.1. We collected color indices of 49 Plutinos as described in Table 7. ¿From the recent analysis of Peixinho et al. (2004) and Doressoundiram (2005), it appears that Plutinos exhibit strong color-color correlations only of the $(B-V)$ and $(V-R)$ indices, pointing to the possibility of different coloring processes acting differently on the Plutinos surface. There is some evidence that small $\left(H_{R}>7.5\right)$ Plutinos are blue while large $\left(H_{R}<7.5\right)$ ones are red as seen by Hainaut and Delsanti (2002) and Peixinho et al. (2004) but not by Doressoundiram et al. (2002). There presently appears no correlation of Plutinos colors with any orbital elements (Doressoundiram et al., 2002, 2005 and 2008).

Present analysis of the colors of objects in other resonances is limited by small number statistics. However the diversity uncovered by Gulbis et al. (2006) is worth pointing out, and certainly warrants further investigation. Unlike the objects in the 3:2 resonance, the majority of objects in the 5:2 resonance are neutral and all but one of the objects in the 4:3, 5:3, 7:4, 2:1, and 7:3 resonances are red. In particular, the objects in the 7:4 resonance are remarkably red, with one object $2001 \mathrm{KP} 77$ reaching an amazing $(B R)=2.24 \pm 0.27$. It 
will be very interesting to see how the present scenario of resonance population can explain such a diversity of colors.

\subsubsection{Scattered KBOs - The scattered disk}

The third dynamical group also known as the scattered disk objects or SDOs and forming the scattered disk is not as strictly defined as the other two groups, and re-classifications are frequent. It is loosely defined as a "torus" with an inner edge near 35-40 AU and extending out to distances of at least several hundreds of AU (Jewitt, 2002a), and composed of objects on orbits with large ranges of eccentricities and inclinations. Morbidelli et al. (2003) have proposed a more formal definition of the scattered disk as "the region of the orbital space that can be visited by bodies that have encountered Neptune within a Hill's radius at least once during the age of the solar system , assuming no substantial modification of the planetary orbits". More recent classification schemes rely on numerical integrations over 10 Myr to determine orbital behavior. Elliot et al. (2005) and Gladman et al. (2008) however still differ on the definition of SDOs, but the divergence concerns only a few objects, and this will not affect statistical analysis.

The existence of this population of scattered planetesimals was hypothesized by Ip (1989), Torbett (1989), as well as by Ip and Fernández (1991) according to scenarios where Uranus and Neptune scattered nearby planetesimals during the late stages of planetary formation over a large range of heliocentric distances thus forming the scattered Kuiper belt and the Oort cloud. As illustrated by Figure 1 of Stern (2003), there may be a continuum of objects from the scattered CKBOs to the SDOs and to the Oort cloud objects which may vastly outnumber the known KBOs. The numerical investigations of Levison and Duncan (1997) and Duncan and Levison (1997) firmly established the ground for the existence of SDOs with subsequent confirmation by the first discovery of $1996 \mathrm{TL}_{66}$ by Luu et al. 
(1997). In the framework of the scenario of Gomes (2003) generalized by the "Nice" model, the SDOs as well as the high-inclination CKBOs and probably the high-inclination plutinos have a common origin as primordial scattered objects originally coming from an inner denser region of the original planetesimal disk around $25 \mathrm{AU}$. The question has however been raised of a continuous flux of objects from the classical Kuiper belt (in contrast with the above primordial origin), either by dynamical mobility within or near the numerous mean-motion resonances or by injection of collisional fragments. The present consensus is that this would result in only a minor contribution, at least for large objects (Morbidelli et al., 2004, Gomes et al., 2008).

There exists objects on high-eccentric, inclined orbits just beyond the $q$-range of objects scattered by Neptune (prominent examples are $2000 \mathrm{CR}_{105}$ and $2003 \mathrm{VB}_{12}$ Sedna) forming the so-called "extended scattered disk" (Gladman et al., 2002; Emel'yanenko et al., 2004) which cannot be explained in the above framework (a stellar encounter may be required). In our study, we do not single out this population, first because too few objects are known and second, because they are likely unrelated to Centaurs and ECs.

The color data used in the present analysis come from our own compilation as described in subsection 3.1.1. We collected color indices of 40 SDOs as detailed in Table 7. The colors of SDOs have been analyzed by Doressoundiram et al. $(2002,2005)$ and Peixinho et al. (2004) but these authors cautioned on possible biases from small number statistics. SDOs are lacking very red colors, a property that they apparently share with the high-inclination population of CKBOs. Peixinho et al. (2004) found strong color-color correlations of all their indices pointing to a single reddening process while Doressoundiram et al. (2005) found limited correlations suggesting several coloring agents. Finally their colors are not correlated with any orbital parameters nor with absolute magnitudes $H$ (i.e., sizes assuming the same albedo for all objects). 


\subsection{Centaurs}

In broad terms, the Centaurs are a transition population of objects between the Trans-Neptunian objects and the Jupiter-family comets. Jewitt and Kalas (1998) proposed a strict definition of Centaurs as objects having $q \geq 5 \mathrm{AU}$ and $a \leq 30 \mathrm{AU}$ pointing out that a more liberal definition with $5 \leq q \leq 30 \mathrm{AU}$ (i.e., excluding the semi-major axis) leads to including Pluto and many Plutinos as Centaurs. In their grand scheme of cometary taxonomy, Levison and Duncan (1997) stated that "a comet that has and $a>a_{J}$ (orbit is exterior to Jupiter) is designated as Chiron-type or a Centaur". Unless they mean by "comet" an object which sport of coma, this definition would include Pluto and all TNOs! As cometary activity may unexpectedly switch on or off, this can not be taken as a solid criterion. A definition involving the Tisserand parameter relative to Jupiter remains however desirable since it allows to put the Centaurs in the family tree of comets proposed by Levison (1996). However, it must be supplemented by a strict constraint on the orbit, either that of Jewitt and Kalas (1998), $q>q_{J}$ and $a<a_{N}$ (consistent with that of the Minor Planet Center, and adopted in this present study), or that of Doressoundiram et al. (2005), $q<a_{N}$ and $Q<48 \mathrm{AU}(2: 1$ resonance).

Most Centaurs are inactive but a few display cometary activity: 2060 Chiron, 166P/ NEAT, 167P/ CINEOS, 60558 Echeclus, 29P/Schwassmann-Wachmann 1, 39P/Oterma, 165P/ LINEAR, C/2001 M10 (NEAT), and P/2004 A1 (LONEOS) [Bauer et al., 2003a; Lawrence et al., 2001]. We note that $29 \mathrm{P}$ with $q=5.7 \mathrm{AU}$ and $a=6.0 \mathrm{AU}$ satisfies the definition of Jewitt and Kalas (1998) but that it has $T_{J}=2.983$ allowing equally a classification as a Jupiter-family comet (see next section).

The color data used in the present analysis come from our compilation of the sources already listed in the above subsections augmented by the results of Brown and Luu (1997), Bauer et al. (2003b), Davies et al. (1998), Jewitt and Luu (2000), Luu and Jewitt (1996), 
as well as Lamy et al. (2007) for 29P. Altogether we collected color indices for 32 centaurs (Table 7).

Tegler et al. (2008) have recently presented a comprehensive review of the properties of Centaurs, and we simply summarize their main conclusions relevant to our present work.

- Centaurs exhibit a unique color property, the bimodality of the distributions of the two indices which involve the B-band, $(B-V)$ and prominently $(B-R)$, not seen in the other indices. Application of the Dip Test (a non-parametric test that does not assume a particular probability distribution and does not require binning data) to their sample of $\mathrm{N}=26(B-R)$ color indices implies that the $(B-R)$ color distribution is bimodal at a confidence level of $99.5 \%$. The gap $1.4<(B-R)<1.7$ divide the Centaur population into a gray group (16 objects) and a red group (10 objects).

- Centaurs with activity have nearly solar colors. A notable exception is 166P/ NEAT which had an unusually red color during a period of activity, (Bauer et al. 2003a).

- There is no statistically significant difference between the orbital elements and absolute magnitudes of gray and red Centaurs except that the red ones have significantly lower inclinations than the gray ones.

- Gray Centaurs exhibit lower albedos than red Centaurs.

Peixinho et al. (2004) have further noted a partial color-color correlations, the $(B-V)$ index being strongly correlated with $(R-I)$ contrary to $(V-R)$, a feature that they share with Plutinos suggesting a possible connection between these two families. Finally, in a near-infrared color survey of 17 Centaurs, Delsanti et al. (2006) found two groupings in their $(H-K)$ vs $(B-R)$ color-color diagram, suggesting a correlation between the absorbers at B-band and H- or K-band. 


\subsection{Ecliptic comets}

Contrary to Centaurs, ecliptic comets are completely defined by their Tisserand parameter, $T_{J}>2$ (Levison 1996). They are subdivided into two groups: the Jupiter-family comets have $2<T_{J}<3$ (but there are a few comets with $T_{J}$ slightly larger than 3 that dynamically belong to the Jupiter family, Duncan et al., 2004) and the Encke-type comets have $T_{J}>3$ and $a<a_{J}$. This latter group has only one active member namely comet 2P/Encke, 107P/Wilson-Harrington (but probably not a comet) and several small asteroids which could well be extinct comets.

In the framework of the evolution described below, ecliptic comets are understood as the "end-state" of Centaurs that are scattered into the inner solar system by Jupiter. Once trapped as a JFC, the median dynamical lifetime to scattering by the terrestrial planets is 0.4 Myr (Levison and Duncan, 1994). ¿From their numerical investigation of the orbital evolution of 53 Centaurs, Tiscareno and Malhotra (2003) found that those which stay in the Jupiter family have a mean dynamical lifetime of 50000 yr. Estimates of the physical lifetime of JFCs ranges from 3000 to $25000 \mathrm{yr}$ with a mean value of $12000 \mathrm{yr}$ according to Levison and Duncan (1997) and down to 2500 yr according to Emel'yanenko et al. (2004); a mean value of roughly $10000 \mathrm{yr}$ is widely accepted. Additional timescales have been presented and discussed by Jewitt $(1996,2002 b)$ : in particular the sublimation lifetime is such that "JCFs smaller than $40 \mathrm{~km}$ should, if in equilibrium sublimation with sunlight, lose all their volatiles before their dynamical evolution is complete". The end-state of JFCs is therefore dead comets, either fully volatile-depleted, wholly refractory bodies or dormant objects whose volatile-rich interior is sealed by a mantle. However comets might disrupt before they reach this end-state, but surviving large fragments if any will follow the above evolution. 


\subsection{Candidate dead comets}

According to the above considerations, dead comets should be abundant. There are growing evidences that asteroid-like objects in comet-like orbits based on their Tisserand parameters are excellent candidates. The color data of such candidates come from the compilation of Jewitt (2002a). Except for 1996 PW, 1998 WU24, 1999 LE31 and 2000 HE46, the color indices were derived from spectra assuming the constant slope tabulated by this author. Not all spectra cover the $B, V, R, I$ range. The spectra extending from $B$ to $R$ were extrapolated to $I$ assuming constant slopes. The color indices of the candidate dead comets lie in rather narrow ranges of moderately red colors (only one blue object). The color-color correlations are quite high as many data points lie along the "reddening lines". This property results from the constant slopes measured by Jewitt (2002a) and our above extrapolation.

\subsection{Jupiter Trojans}

The Jovian Trojans, asteroids confined to two swarms at the L4 and L5 Lagrangian points of the Sun-Jupiter system, were long thought to have accreted very early from near-Jupiter planetesimals, possibly during the final growth period of the planet (e.g., Shoemaker et al., 1989; Peale, 1993; Kary and Lissauer, 1995; Marzari and Scholl, 1998; Fleming and Hamilton, 2000). Trojans could therefore represent a small fraction of the population of planetesimals which formed among the giant planets, the bulk of them having been ejected from the solar system. A few percent got captured into the Oort cloud where they remain until perturbations from passing stars, molecular clouds, or the galactic tide send some of then into the inner Solar System where they appear as Nearly-isotropic Comets (NICs). The process that led to the stabilization of these objects in weakly bound orbits about L4 and L5 is however still unclear and the above scenario cannot explain some 
basic properties of the Trojan population, notably its broad orbital inclination distribution. These difficulties are alleviated by the Nice model where Trojans are formed in more distant regions and subsequently captured into co-orbital motion with Jupiter during the time when the giant planets migrated (Morbidelli et al., 2005). This capture took place during a very short time, just after Jupiter and Saturn crossed their mutual 1:2 resonance, but before Trojans evolved through a large eccentricity phase that brought many of them in the inner solar system with perihelia as low as 2 to $3 \mathrm{AU}$. Interestingly enough, this scenario places the origin of Trojans in the planetesimal disk that drove planetary migration, and suggests that the bulk of them have undergone orbital evolutions similar to that of JFCs and some Centaurs that brought them in the inner solar system, thus experiencing similar thermal evolutions.

Calculations of collision rates indicate that the Trojans, by virtue of low number density and low relative velocity, have only a few percent of the rate of collisions presently experienced in the main belt. These numbers suggest that the Trojan populations are not fully evolved in terms of collisional fragmentation, and raise the possibility of survival of some members from their initial accreted stage. Jewitt et al. (2000) studied the size distribution of a large number of Trojans and found two regimes characterized by two distinctly different values of the differential power-law index $q$. Trojans smaller than $r_{c} \sim 30 \mathrm{~km}$ have $q=3.0 \pm 0.3$ consistent with the slope expected from a collisionally relaxed population $\left(q \sim 3.5\right.$, Dohnanyi, 1969). Trojans larger than $r_{c}$ have $q=5.5 \pm 0.9$ and are considered to be more "primordial". These results are entirely consistent with the collisional model of Marzari et al. (1997).

The color data come from the work of Fornasier et al. (2007) and their compilation of previously published data resulting in a large sample of 85 Trojans. The L4 and L5 groups share similar optical properties (color distributions, low albedos) and in fact, Trojans are 
known to be dominated by D-type asteroids, with the exception of the Eurybates family in the L4 swarm, where there is a dominance of C- and P-type asteroids.

Both Jewitt and Luu (1990) and Fitzsimmons et al. (1994) have noted a color-diameter trend among Trojans and D-type asteroids with the smaller objects being redder than the large ones. This is somewhat surprising since the measured objects have radii larger than $r_{c} \simeq 30 \mathrm{~km}$ (except for a couple) implying that they all belong to the same "primordial" population. Based on their much larger data set, Fornasier et al. (2007) did not find such a correlation, and further found no correlation between colors and orbital elements, with the exception of the Eurybates family (their color trend is opposite to that found in the Kuiper belt).

\subsection{Dynamical relationships}

The early speculations on the existence of a belt of icy objects beyond the orbit of Neptune were tightly connected to the question of the origin of the ecliptic comets (Edgeworth, 1949; Fernández, 1980). The first dynamical simulations by Duncan et al. (1988) showed that a source beyond Neptune with a low initial inclination distribution is consistent with the observed distribution of orbits of ECs. Levison and Duncan (1997) have subsequently presented a comprehensive dynamical simulations of objects leaking from the Kuiper belt. They were initially in low-eccentricity, low-inclination orbits in the Kuiper belt, that is in the "cold" population of CKBOs to use the present terminology. The bulk of the objects that keep evolving inward from the Kuiper belt become visible Jupiter-family comets (JFCs) with dynamical lifetimes of $\sim 0.4$ Myr. Another possible scenario relies on the importance of collisions in the evolution of the Kuiper belt (Stern, 1995). The extra velocities gained by the collisional fragments may kick them out into the strongly chaotic regions of the belt, and then to dynamically evolve to Centaurs and ECs (Farinella and 
Davis, 1996). Davis and Farinella (1997) estimated that the two processes, diffusion and collision, should roughly produce the same number of ECs. There is however a fundamental difficulty with the classical Kuiper belt being the dominant source of the Centaurs and ECs as it would require that the number of objects in the belt be more than ten times the number in the scattered disk to offset the large ratio in their dynamical lifetimes (Duncan et al., 2004). Since the two populations of $100-\mathrm{km}$ objects are found to be comparable (Trujillo et al. 2000), the more than tenfold requirement would violate the observational evidence on the assumption that these two populations have similar size distributions. Emel'yanenko et al. (2004) have further argued that the observed distribution of near-Neptune objects is very different from that predicted by a model in which the majority of the JFCs originate from non-resonant CKBO orbits. Finally the dynamical simulations of Levison and Duncan (1997) have shown that once an object evolved out of the Kuiper belt, it spends 90\% of its time as a SDO before becoming a Centaur.

An origin of Centaurs and ECs in the scattered disk as the primary source has therefore been favored since the requirement of $6 \times 10^{8} \mathrm{SDOs}$, that is 0.004 Earth mass (Duncan and Levison, 1997) is well compatible with our present knowledge of this disk, and since dynamical pathways are understood (Duncan and Levison, 1997; Emel'yanenko et al., 2004; see also Fig. 3 and Plate 7 in Duncan et al., 2004). Levison and Duncan (1997) have shown that candidate Centaurs initially spend considerable time in the scattered disk, but once their perihelions drop to Uranus' location, they follow an inward or outward random walk between planets in a series of handoffs. These objects tend to be under the dynamical control of just one giant planet, handing them off to the neighboring planet, either interior or exterior to it. Centaurs which are scattered into the inner solar system by Jupiter (about half of them) become JFCs if they develop a coma. Numerical investigations were later performed by Tiscareno and Malhotra (2003) who studied the dynamical behavior of 53 known Centaurs over 100 Myr and Horner et al. (2004a,b) who considered 23000 clones 
of 32 Centaurs over 3 Myr. They confirmed the above strongly chaotic nature of their orbits: about two-thirds of these Centaurs will be ejected from the solar system (or will enter the Oort cloud), while one-third will become JFCs. The work of Horner et al. (2004b) reveals a rich variety of orbital evolutions including episodes of short-period comet behavior, temporarily capture into the Jovian Trojans, temporarily capture into mean-motion and Kozai resonances, and traversals of the entire solar system. Examples of outward migration were often found in their simulations and one particular clone of Chiron moves all the way into Earth-crossing and then all the way back out to beyond Saturn. Bailey and Malhotra (2008) investigated the Centaur-comet transition in more detail by numerically following the dynamical evolution of 62 known Centaurs over 30 Myr. They confirmed that a majority of them $(\sim 80 \%)$ follows the above pattern of successive planetary hand-offs, and spends a few hundred thousand years as JFCs, comparable to the typical dynamical lifetimes of JFCs $\left(\sim 10^{5}\right.$ years $)$. The orbital evolution of the other $\sim 20 \%$ is characterized by hopping between mean motion resonances with Uranus and Neptune, and those Centaurs spend no or little time as JFCs.

There is however one critical aspect in the SDO-Centaur link, namely the extrapolation of the size distribution of SDOs presently known to a diameter of approximately $100 \mathrm{~km}$ to smaller sizes down to those of cometary nuclei, typically a few $\mathrm{km}$. As long as a single power law extrapolation is adopted, the above link appears secure. However the deep HST survey of Bernstein et al. (2004) shows that the brightness distribution of KBOs flattens significantly at magnitudes fainter than $m \simeq 24$ (roughly equivalent to a diameter of $200 \mathrm{~km}$ ). The implication is that the population of small KBOs, down in the size regime of JFCs, is far smaller than that estimated from extrapolation of the size distribution of larger KBOs. Using this new constraint and a 4 gigayear numerical simulation together with an improved model for the intrinsic orbital distribution of the scattered disk, Volk and Malhotra (2008) concluded that the influx of SDO objects required to replace the comets 
at a rate of $3.310^{-} 2 \mathrm{yr}^{-} 1$ so as to maintain the current population falls short by two orders of magnitude, and that alternative sources must be reconsidered, the CKBOs, the resonant KBOs, or the Jupiter Trojans.

Regarding the CKBOs, Volk and Malhotra (2008) noted that this population has a faint-end size distribution which may be significantly steeper than that of the SDOs (Bernstein et al.2004), and its fractional escape rate (Duncan et al. (1995) is only a factor $\sim 3$ less than what they found for the SDOs, thus opening the possibility that CKBOs are a viable source worth re-assessing.

Resonant KBOs, and particularly Plutinos, have already been considered as a possible source of Centaurs and ecliptic comets in the past. To become so, Plutinos must first be destabilized and experience close encounters with Neptune, i.e., their perihelia must decrease to $\sim 30 \mathrm{AU}$. The specific case of the 2:3 resonance has been numerically investigated by Morbidelli (1997). Orbits at the border zone between the unstable and stable regions are characterized by chaotic diffusion. The orbits may diffuse for several billion years, then leak out of the border zone and enter the strongly chaotic region where an encounter with Neptune occurs very quickly. Alternatively, collisional fragments cloud be directly injected into the strongly chaotic zone. Another mechanism has been suggested by Yu and Tremaine (1999) in which Pluto itself might scatter Plutinos with specific eccentricities into unstable orbits although Petit et al. (1999) argued that Pluto has too small a mass to do so.

Regarding the Jupiter Trojans, the collisional model of Marzari et al. (1997) reveals an interesting behavior: some collisional fragments can be destabilized from the lagrangian stable regions and ejected on comet-like orbits, a result independently confirmed by Levison et al. (1997). These authors estimated that this mechanism could supply $\sim 10 \%$ of the current population of ecliptic comets and Centaurs. In this context, it is interesting to note that Jewitt and Luu (1990) have illustrated the very close correspondence between the 
visible spectra of Trojan 1988 BY1 and the nucleus of comet P/Tempel 2, which raises the possibility of the latter being a former Trojan.

The dynamical lifetime of Centaurs is equal to the timescale for transport from the Kuiper belt to Jupiter crossing orbits and ranges from 1 to 100 Myr (Dones et al., 1996; Tiscareno and Malhotra, 2003; Horner et al., 2004a), although some can take much longer than that (Duncan and Levison, 1997). This is sufficiently short that mutual collisions are excluded (Durda and Stern, 2000). But during their dynamical lifetime, Centaurs suffer many close encounters with the four outer planets, for instance $\sim 8000$ in the case of the 53 Centaurs studied by Tiscareno and Malhotra (2003) with a few percents impacting the planets. Tidal break-up similar to that experienced by comet Shoemaker-Levy 9 could occur, and enhance the number of comet progenitors over what is predicted by steady state dynamical models (Volk and Malhotra, 2008).

\subsection{Color properties and relationships}

\subsubsection{The histograms of the colors}

Figure 10 displays the histograms of the color indices of the eight families of objects introduced in the above sections (excluding the NICs) and roughly arranged in order of decreasing reddening (from top to bottom), while preserving suspected relationships. Also displayed are the associated mean values and standard deviations. The distributions of $(B-R)$ clearly show the bimodality of Centaurs and, to a lesser extent of SDOs. It is not really apparent for KBOs and totally absent for the nuclei of ECs and for the candidate dead comets. In contrast with the KBOs, the Centaurs and the nuclei of ECs, the candidate dead comets and the Trojans have a much narrower color range. 


\subsubsection{Color-Color Diagrams}

Figure 11 displays the five color-color diagrams similar to Fig. 4 but for the nine populations of objects, i.e., including the NICs. Error bars are not included to allow full legibility of the figures. They can be found in Fig. 4 for cometary nuclei and in similar diagrams already published for the other objects (e.g., Hainaut and Delsanti, 2002). A striking feature is that all populations tend to cluster around the "reddening lines". The scatter around these lines depends both upon the population and the color index, which makes the interpretation quite difficult. We note that it is most pronounced for the CKBOs and the cometary nuclei. There exists a systematic effect in the diagrams containing the data in the $I$ band, i.e., the $(R-I)$ and $(V-I)$ indices, already pointed out by Hainaut and Delsanti (2002), an increasing deviation from the "reddening lines" as the reddening increases. As explained by these authors, this corresponds to the fact that the spectrum of the reddest objects flattens toward the infrared, where it is typically flat/neutral (Davies 2000; McBride et al., 1999). Conservely, the $(V-R)$ versus $(B-V)$ diagram exhibits the least derivation and also the least scatter from the "reddening lines".

Table 8 presents a quantitative assessment of these color-color relationships based on the Spearman rank correlation $\rho$ and the associated significance level (SL). The families of CKBO-LI and ECs clearly stand out as presenting the weakest color-color correlations and sometime anti-correlations, a property already perceived on the color-color diagrams. Next come the CKBO-HI with moderate correlations The other three families, Plutinos, SDOs and Centaurs, display pronounced correlations for all combinations of indices. We emphasize the very close resemblance of the correlations for the Plutinos and SDOs and, to a lesser extent, with those of the Centaurs. Consequently, and contrary to the results of Peixinho et al. (2004), color-color correlations do not favor one possible origin of the Centaurs (Plutinos) against another (SDOs). However they do support a common origin of 
the Plutinos and SDOs.

\subsubsection{Color-size relationship}

Jewitt and Luu (2001) first reported that the color-size relation of KBOs remains inconclusive. There now seems to be a consensus that the larger Plutinos are redder than the small ones (Hainaut and Delsanti, 2002; Peixinho et al, 2004). The case of CKBOs remains confused because of possible multiple correlations between colors and perihelion distances, inclinations and sizes. If Levison and Stern (2001) and Trujillo and Brown (2002) are correct, then the "hot" population contains larger bodies than the "cold" one and therefore those smaller bodies are redder than the larger ones. However Doressoundiram et al. (2002) and Peixinho et al. (2004) disagree as they found that the two populations have the same distributions of absolute magnitudes, that is sizes assuming the same albedo for all objects. In their recent review of colors of TNOs Doressoundiram et al. (2008) concluded that no significant trend has been found between color and size, or absolute magnitude.

\subsubsection{The color-distance relationship}

Figure 12 displays the $(V-R)$ indices of the minor objects as a function of their semimajor axis and is intended to offer a global view of the colors in the solar system. For that purpose, we included two additional populations, main-belt asteroids and Damocloids. Like TNOs and Trojans, main-belt asteroids formed in the early solar system from materials that failed to build large planets, and contain the signature of primordial processes (Farinella et al., 2000). They are the sources of transient populations, JFCs from the TNOs (and possibly also from the trojans), near-Earth asteroids and meteoroids from the asteroid belt. Included in Fig. 12 are the colors of 23 asteroids in the 3:1 jovian mean 
motion resonance (semimajor axis in the range $2.46 \leq a \leq 2.54 \mathrm{AU}$ ) obtained by Luu and Jewitt (1990b) from high-quality CCD spectra. These asteroids are considered to be one of the source regions of the near-Earth objects. The Damocloids are objects thought, on dynamical grounds, to be inactive Halley-family and long-period comets. The color data of twelve of them have been reported by Jewitt (2005).

Striking features apparent in Fig. 12 are the wide diversity of colors shared by KBOs, SDOs, Centaurs and cometary nuclei. The ranges of colors for the other families appear more restricted. Trojans are a distinct group of red objects while the colors of the main-belt asteroids range from slightly blue to moderately red $[0.23<(V-R)<0.49]$. In fact, the most common blue objects are these asteroids and the cometary nuclei; there are just a few TNOs, one Trojan and one dead comet sharing this property.

Regarding the individual families of TNOs, the color-distance trend has been reviewed by Peixinho et al. (2004). While the CKBOs globally do not show a color-semimajor axis relation, the larger objects do exhibit a strong correlation, the colors increasing with the semimajor axis. This correlation is even stronger with the perihelion distance and has its origin in the "hot" population; it would be consistent with the fact that this population could contain larger bodies than the "cold" one (Levison and Stern, 2001; Trujillo and Brown, 2002, 2003), but as discussed in section 3.8, both Doressoundiram et al. (2002) and Peixinho et al. (2004) do not support this view. These latter authors further noted a quasi dichotomic behavior of colors around $q \sim 38 \mathrm{AU}$ which hints for a masking of the color-perihelion correlations over the color-inclination trend. As for the other families, Plutinos and SDOs, there is a general agreement on the absence of color-distance correlation (Doressoundiram et al., 2008)

Regarding the Centaurs, and based on the $(B-R)$ index, Tegler et al. (2008) found that red objects have slightly larger orbits and extend farther from the Sun than the gray 
ones (there are no color-distance correlation within a color group). This trend is confirmed by Bauer et al. (2003b) on the basis of correlations of the $(V-R)$ and $(R-I)$ indices with the semimajor axis for the red Centaurs.

To be complete, we recall our result from section 2.6 that the $(V-R)$ index of the red cometary nuclei appears to increase with increasing perihelion distance, but that the Spearman test was inconclusive.

\subsubsection{The color-inclination relationship}

The color-inclination relationship is by far the most undisputed with the following main conclusions. Plutinos, SDOs and Trojans (except the Eurybates family) show no correlation between color and inclination. The correlation is statistically significant for the CKBOs to the point that one can isolate two subpopulations, the low-inclination CKBOs (CKBO-LI)with uniform very red colors, and the high-inclination CKBOs (CKBO-HI) with a broad range of colors, from blue to red. Likewise, the red Centaurs have significantly lower

inclinations than the gray ones. A consistent trend of decreasing reddening with increasing inclination has been noted for cometary nuclei (with the exception of the $(R-I)$ index, see Fig. 6). The chaotic evolution of Centaurs and ECs precludes connecting present to past (before their diffusion) orbital parameters. However inclination may be the one orbital parameter that retains some memory of the past (Levison and Duncan, 1997) making the above color-inclination trend of particular value in understanding the origin of Centaurs and ECs and their connection. 


\section{IMPLICATIONS OF COLOR FOR THE RELATIONSHIPS BETWEEN THE POPULATIONS OF MINOR BODIES}

In order to investigate the question of the possible implications of color for the relationships between the populations of minor bodies, we introduce the cumulative distribution functions (CDFs) of the four color indices, in short cumulative color distributions (CCDs), which are less prone to artifacts associated to histograms. We extensively make use of the Kolmogorov-Smirnov (K-S) test to quantify the probability $P_{K S}$ that two distributions are drawn from a single parent distribution and summarize below several general considerations on this test borrowed from the presentation of Press et al. (1992). It should be kept in mind that one can never prove that two data sets come from a single distribution. Disproving the null hypothesis that two data sets are drawn from the same population distribution function in effect proves that the data sets are from different distributions. Failing to disprove the null hypothesis only shows that the data sets are consistent with a single distribution function. The K-S test offers a simple measure of the overall difference between two cumulative distribution functions as the maximum value $D$ of the absolute difference of distances between these two functions. Its main advantage is to allow expressing the significance level of an observed value of $D_{o b s}$ (as a disproof of the null hypothesis that the distributions are the same) as the probability $P_{K S}\left(D>D_{o b s}\right)$. A probability (significance level) less than (more significant than) some fixed value, typically 0.02 , establishes to near-certainty that the two populations are drawn from different distributions. Its main disadvantage is that it is most sensitive around the median values of the CDFs being thus good at finding shifts but not spreads which may affect the tails of the CDFs but leave their median values unchanged. A test proposed by N.H. Kuiper (not Gerald) does guaranty equal sensitivities at all values but requires reparametrization of the variable on a circle which is not really appropriate to color indices. The K-S test is available for one sample (e.g., one color index) and two samples (e.g., two color indices) but has 
not been generalized to larger samples. Therefore the K-S test cannot be simultaneously performed on the four color indices $(B-V),(V-R),(R-I)$ and $(B-R)$.

\subsection{Cumulative Distribution Functions}

Figure 13 displays the cumulative distribution functions of the eight families of objects introduced in section 3. Let us first comment the CCDs of the $(V-R)$ index which incorporate the largest number of objects (notably EC nuclei). Proceeding in order of decreasing reddening, we can delineate: i) the CKBO-LI which clearly stand out as a distinct, very red family (to be later referred to as TNO-I), ii) a group composed of the CKBO-HI, the Plutinos and the SDOs (to be later referred to as TNO-II) and the Centaurs whose CCDs are intermingled, iii) the cometary nuclei, iv) the Trojans, v) the candidate dead comets.

This progressive and systematic reddening trend possibly connected to the evolution of these families of objects, will be discussed later. To a large extent, the CCDs of the $(R-I),(B-V)$ and $(B-R)$ indices confirm the above hierarchy; those involving the $B$

band, and particularly the CCDs of the $(B-R)$ index further exhibit a spread in the colors that clearly reveals two important features: i) the bimodality of the color distribution of Centaurs, ii) the closeness of the CDDs of the CKBO-HI and Plutinos which both tend to separate from that of the SDOs.

Finally note that the CCDs of ECs, Trojans and candidate dead comets of all color indices are well separated.

In view of the above results, we performed an experiment to test whether we could obtain even more legible results amenable to interpretation. We temporarily merged the CKBO-HI, Plutinos and SDOs into a single group named "TNO II", and we split the 
family of Centaurs according to their bimodal distribution in Centaurs I with $(B-R)>1.7$ and Centaurs II with $(B-R)<1.4$ respectively corresponding to the red and gray groups introduced by Tegler et al. (2008). Strictly speaking, it is mathematically invalid to divide a population in half based on color and then use the color distributions of the resulting subpopulations in statistical comparisons. There are however several strong arguments to attempt this division: i) the $(B-R)$ color distribution exhibits two well defined components with a gap $1.4<(B-R)<1.7$ much larger than the width of the two individual distributions; ii) there is by far no overlap between these two distributions, even taking into account the uncertainties; iii) Tegler et al. (2008) have now established that the two subpopulations have very different inclinations, thus offering an independent basis for the division similar to the CKBOs LI/HI dichotomy. We note that Schaefer et al. (2008) have proposed a division of the icy bodies in the outer solar system in five groups which separates the red and gray Centaurs, and puts them into different groups. This division could well reflect different physical evolutions between those which have never visited the inner solar system and those which on the contrary have done so, consistent with the results of dynamical integrations presented in section 3.2. In fact, a K-S test of the resulting CDCs yields a probability $P_{K S}$ so low that it establishes to near-certainty that the two populations would be drawn from different distributions as far as color is concerned.

The following new features emerge from inspection of Fig. 14.

- Centaurs I now appear as the reddest population of all minor bodies in the outer solar system.

- Based on the CCDs of the $(V-R)$ index, the classification in order of decreasing reddening is now: Centaurs I, TNO I (= CKBO-LI), TNO II, ECs, Centaurs II, Trojans and candidate dead comets.

- This hierarchy holds for the other color indices as well with one notable exception, 
the CCD of Centaurs II shifts position between that of the SDOs and that of the candidate dead comets. Curiously enough, the CCD of Centaurs II is close to that of the Trojans for the $(B-R)$ index, and close to that of the candidate dead comets for the $(B-V)$ index.

\subsection{Comparison of the Cumulative Color Distributions}

Figure 15 presents a detailed and quantitative comparison of the cumulative color distributions of the populations of minor bodies on the basis of their $(V-R)$ color index. We compare two populations at a time, first displaying their original CCDs (left panels) and subsequently the shifted CCDs that maximize the Kolmogorov-Smirnov probability $P_{K S}$ (central panels). The right panels display the probability $P_{K S}$ as a function of the color shift. Similar figures have been generated for the other indices but are not displayed for concision. The results are then summarized in Table 9 which further presents the probabilities calculated from the two-dimensional K-S test. We note that most combinations need significant shifts for the respective CCDs to match, a result confirmed by the very low probabilities reported in Table 9. There are three exceptions, the Plutino - Centaur, the SDO - Centaur and the CKBO-HI - Centaur connections with respective shifts of the $(V-R)$ color index of 0.009, 0.002 and 0.021. The Plutino - Centaur connection has often be pointed out by several authors (e.g., Peixinho et al., 2004). The other two connections, SDO - Centaur and CKBO-HI - Centaur look quite interesting when looking for a possible parent-child relationship, but we know from Fig. 13 that the CCDs of the $(B-R)$ index would obliterate this possibility because of the bimodality inherent to the Centaurs. Beside the $P_{K S}$ probabilities associated to the $(V-R)$ index, although substantially larger than the other connections, never reach levels which would insure a statistically significant result.

We now examine the impact of considering the two populations of Centaurs as 
introduced in section 4.1. Table 10 reveals a clear-cut situation where the probabilities are so low (with a few isolated exceptions) that all connections can be rejected. If the reality of two groups of Centaurs is accepted then we have to conclude that the above putative connections are fortuitous, the color properties of the mixed Centaur groups happening to match those of Plutinos, SDOs and CKBO-HI by chance.

\subsection{Global view of the colors of primitive minor bodies in the solar system}

Figure 16 attempts to summarize the current situation of the colors of primitive minor bodies in the solar system based on the difference of colors using our large data set. The differences between the respective cumulative distribution functions of color indices were determined using two estimators, i) the difference between the means of the distributions taking that of the CKBO-LI as a reference, and ii) the color shifts that maximize the K-S probability of any combinations (as determined in section 4.2) using the CKO-LI as a reference for the other TNOs and the Centaurs, the latter as a reference for the EC, and the ECs as a reference for the candidate dead comets. The two estimators lead to consistent pictures, except in a few cases. We have further considered the Centaurs both as a single group and as a dual group as defined in section 4.1, and displayed them with the first estimator. When comparing the properties of these primitive bodies, one should keep in mind the enormous difference in sizes between the TNOs measured so far (typically $>100 \mathrm{~km}$ ) on the one hand and ECs and candidate dead comets on the other hand. We found no color-size relationship for any family, with the possible exception of the Plutinos, but the absence of a relation in the existing data is not a formal proof against its existence. The $(V-R)$ index presents a clear cut picture with well-defined and systematic blue shifts from the CKBO-LI to the group CKBO-HI + Plutinos + Centaurs + SDOs $[\Delta(V-R) \simeq-0.1]$, to the ECs $[\Delta(V-R) \simeq-0.08]$ and to the candidate dead comets 
$[\Delta(V-R) \simeq-0.06]$, the Trojans falling in between these latter two families. The $(R-I)$ and $(B-V)$ indices present pictures fully consistent with the above behavior; we can just remark that the ECs tend to join the group CKBO-HI + Plutinos + Centaurs + SDOs in the case of the latter index, the mean estimator even placing them in between the Plutinos and the SDOs.

When considering the two groups of Centaurs, Centaurs I stand out as the reddest population for all indices, but the color shift with respect to the TNOs is found to be color dependent. Conservely Centaurs II are bluer than the TNOs, and further display an interesting color dependent behavior, becoming progressively redder when going from the $B$ to the $V$ and to the $R$ bands. Their $(B-V)$ index lies between those of the candidate dead comets and the Trojans (in fact very close to the former), their $(V-R)$ index between the Trojans and the ECs, and their $(R-I)$ between the ECs and the SDOs.

\section{DISCUSSION}

Recently several authors have overemphasized the importance of elaborated statistical tests (e.g., Hainaut and Delsanti, 2002; Peixinho et al., 2004; Meech et al. 2004). While it is certainly required that statistical analysis be properly performed, even the most elaborated tests have their own limitations and biases (c.f. our discussion of the Kolmogorov-Smirnov test in section 4). We further wonder whether this is presently the real issue in view of several major difficulties.

i) The classification of primitive bodies is not always firmly established. In addition, an "apparent" family defined on some dynamical criteria may in fact mixes two or more groups with different origins and physical properties. Although not directly relevant to our present study, the case of near-Earth objects provides a good illustration with 
a mix of asteroids and evolved comets. Here again, the present definitions of the family of Centaurs may hide very different evolutionary paths of utmost importance for their physical properties.

ii) Obtaining accurate color measurements of very faint KBOs and active cometary nuclei still remains a challenge, and the resulting uncertainties are sometime large as pointed out in our analysis. In this respect our technics of cometary nuclei detection exploiting the superior spatial resolution of the Hubble Space Telescope opens the possibility of characterizing the bulk of them irrespective of their activity, and to reach high signal-over-noise ratios since they are often observed at minimum geocentric distances.

iii) Last but not least, the representativity of the available data sets (i.e., completeness) remains a key issue, and we have enlighted its impact on the cometary nuclei. We believe that it presently constitutes the most severe limitations, far more important than the statistical tests themselves.

In view of the above difficulties, one may wonder whether the strict application of the K-S test to the CCDs is appropriate. While disproving the null hypothesis is not a problem, it is unlikely, except if we are extremely lucky, that we will ever prove it with significance levels high enough according to the statistical standards. We probably should satisfy ourselves by comparing our still imperfect CCDs, and conclude whether they are consistent or not. This is precisely the role of Fig. 15, and the quantification of the color shifts to be compared with the typical uncertainty of the color measurements may in fact offer a far more robust test of consistency than the strict application of the K-S test.

Although the diversity of colors of the cometary nuclei has already been perceived even on a small sample (Luu, 1993), the extremely broad range of colors uncovered by our observations with the HST is totally unexpected and, we believe, of major significance for understanding the origin and evolution of ecliptic comets. Our study invalidates previous 
conclusions biased by too small data sets, namely that comets colors are significantly bluer than those of KBOs and Centaurs (Hainaut and Delsanti 2002), that wide diversity of colors is peculiar to outer solar system objects not observed among cometary nuclei (Doressoundiram et al., 2002), that ultrared matter present in those objects is missing in cometary nuclei whose color distribution is indistinguishable from that of candidate dead comets (Jewitt 2002a), and that the average colors and overall color distributions of Trojans (excluding the Eurybates members) are not distinguishable from that of the ecliptic comets (Fornasier et al., 2007). Jewitt (2002a) defines ultrared matter as having a normalized reflectivity gradient larger than $25 \%$ per $100 \mathrm{~nm}$, that is $(V-R)>0.63$. Five of our observed nuclei, 50P, 71P, 86P, 110P and 137P exceed that limit, and three others, $44 \mathrm{P} 59 \mathrm{P}$ and $94 \mathrm{P}$, come very close with $(V-R)=0.62$. Based on this color index, the nucleus of $81 \mathrm{P}$ appears as one of the reddest minor object of the solar system. Our sample does however include a few nuclei which are bluer than the bluest TNO, although not by much if we exclude $14 \mathrm{P}$ whose peculiar color certainly needs confirmation [note also the very large uncertainty affecting its $(V-R)$ index]. ECs lack strong color-color correlations, a property that they share with the CKBO-LI, and which contrasts with the other TNOs and Centaurs.

Our in-depth analysis of the colors of over 370 minor bodies of the solar system indicates significant differences between the cumulative color distributions of the different families of objects. There are exceptions concerning the CKBO-HI, the Plutinos, the SDOs and the Centaurs (Fig. 15), although strictly speaking the relevant probabilities never reach levels which would insure a statistically significant result. When attempting to explain the color differences between the different families, we have to keep in mind that it is the surface of the bodies which are observed and that color effects likely result from both differences in pristine composition (resulting from formation at different places in the protoplanetary disk) and differences in their evolution (resulting from different processes). 
As pointed out by Grundy (2009), there exists serious arguments in favor of colors being related to primordial bulk composition, rather than being just an environmental surface effect: the absence of color dependence upon size (e.g., Doressoundiram et al. 2008), the rarity of color lightcurve variations (e.g., Jewitt and Luu 2001; Doressoundiram et al. 2005), and the highly correlated colors of binaries (Benecchi et al. 2008). The most plausible explanation relies on the presence of red, complex organic compounds such as tholins resulting from the irradiation of ices by cosmic rays, ultraviolet light and solar wind particles. Another mechanism based on the chemistry of methane as described in the same words by Doressoundiram et al. (2008) and Tegler et al. (2008) involve different condensation processes, as a water-ice rich clathrate within $\sim 40$ AU and as pure methane ice beyond (Lewis, 1972), with subsequent alteration producing compounds with different colors, gray and red. However, in the present dynamical scenarios, the protoplanetary disk is truncated at roughly 30-35 AU (Levison et al., 2008), thus the above dual process could only take place after the formation of the TNOs and their sweeping away to their actual location. It obviously cannot explain the different color properties of TNO families located beyond $\sim 40 \mathrm{AU}$ such as CKBO-LI and CKBO-HI. In addition, it would only affect their surface and not their interior. Whereas the arguments in favor of the composition are strong, evolutionary changes as objects approach the Sun must take place to explain the observed differences in colors in the case where a genetic relationship among objects is widely accepted, for instance between the Centaurs, ECs, and candidate dead comets (in principle, they could also result from different sizes, but the available data do not support this alternative).

The rather coherent and progressive shifts exhibited by the color indices (Fig. 16) can be interpreted in the framework of the following (non-unique) scenario which invokes both compositional end evolutionary causes for color differences, and is based on the widely accepted paradigm that reddening is a function of heliocentric distance. 
- The color differences between the different families of TNO result from their formation and/or their evolution at different places in the solar system. This requires that the CKBO-HI, the Plutinos and the SDOs (i.e., the TNO-II using our terminology) formed closer in than the CKBO-LI, a picture remarkably consistent with the scenario proposed by Gomes (2003) and generalized by the "Nice" model. Accordingly the CKBO-LI formed in the outer part of the disk. The colors also support the formation of SDOs at slightly shorter heliocentric distances than the CKBO-HI and the Plutinos, possibly explaining their more efficient scattering. The fact that the colors of the Plutinos is consistent with those of the CKBO-HI, but not with those of the CKBO-LI further strongly supports Gomes' mechanism for populating the resonances over that proposed by Malhotra $(1995,1998)$.

- The colors of Centaurs still present a challenge. Ignoring the color dichotomy for a moment, and considering them as a single group, the similarity of their colors with those of the SDOs is fully consistent with the genetic relationship established by Levison and Duncan (1997) on dynamical grounds. The fact that their colors are further similar to those of the CKBO-HI and of the Plutinos cannot be used to argue one way or another. Once again, it is consistent with the scenario proposed by Gomes (2003) where the TNO-II formed in the same region of the disk, and later ended up in three families (CKBO-HI, Plutinos, SDOs) that are now dynamically isolated from one another. However we must face the reality that there are two groups, Centaurs I (red) and II (gray), and seriously consider the possibility of two separate source regions, or of radically different dynamical evolution, or a combination of both alternatives. At this stage, it is worth recalling that half to two thirds of the inbound Centaurs are scattered back outward, mostly by Jupiter, or in other words, that some Jupiter-family comets evolve back into Centaurs. Examples of outward migration were often found in the numerical simulations of Horner et al. (2004b), and one case is 
known: 39P/Oterma moved its perihelion distance from that of a Jupiter-family comet at 3.4 AU to that of a Centaur at 5.47 AU following a close encounter with Jupiter in 1963. However the process is known to be rather inefficient with a majority of objects being scattered out of the solar system; in addition, it is generally impossible to determine from numerical simulations whether a given Centaur was an EC comet in the past because of their chaotic dynamical evolution. As already pointed out, orbital inclination may retain some memory of the past evolution, and precisely Centaurs II have significantly larger inclinations that Centaurs I, possibly suggesting that the former are more dynamically evolved ("older") than the latter. In view of these evidences, Centaurs II could well represent an evolved population which would have lost their red matter by some process during their inward travel and residence in the inner solar system, very much like ECs, Trojans and candidate dead comets. Centaurs II would naturally originate from the trans-neptunian region, but it is not possible to specify from which family(s) on the basis of the color information alone. There are of course difficulties with this scenario, and in particular it remains to be seen whether the dynamics allows the residence time of these objects as Centaurs II to explain their number in steady state, and whether there are any observational biases favoring their discovery with respect to the Centaurs I.

Centaurs I now appears as the reddest family in the solar system. However the difference of the mean values of its CCD for the $(V-R)$ index with that of the CKBO-LI amounts to only 0.03 (Fig. 14); it is somewhat larger for the other indices, 0.08 for $(B-V)$ and 0.1 for $(R-I)$. There is consequently a large overlap between the range of colors of Centaurs I and CKBO-LI, the former lacking the bluest members of the latter, and having a slight additional fraction of redder members. This opens the possibility that the main source of Centaurs I lies in the classical Kuiper Belt as independently suggested for the JFCs by Volk and Malhotra (2008), and we concur 
with them that this possibility is worth re-assessing. It would then remain to be understood why the dynamics favors the reddest objects, and to investigate whether there exists a yet unstudied family of ultra red objects. According to the adopted paradigm, they would form at the outer edge of the protoplanetary disk.

- Trojans have formed according to the scenario of Morbidelli et al. (2005), i.e., in the trans-neptunian disk, and their dynamical evolution have brought them in the inner solar system with perihelia as low as 2 to $3 \mathrm{AU}$. They have consequently all undergone nearly the same physical evolution, rather similar to Centaurs II, ECs and candidate dead comets. Therefore their colors are relatively homogeneous and their distributions is close to those of the above three families.

- ECs have lost part of their organic red matter, the end-state being reached by the candidate dead comets. This process in fact involves different red compounds ("coloring agents") having for instance different thermal instabilities and different optical properties, thus explaining the different behaviors of the color indices. We emphasize that the mechanisms relying on the formation of mantles (see below) would imply systematic color shifts and not differential color shifts as observed.

- The nuclei of nearly-isotropic comets and the Damocloids share the same properties of being slightly redder than the Sun, and color ranges much narrower than those of ECS [only 0.17 for the $(V-R)$ index] thus excluding very red colors. This is consistent with their origin in the Oort cloud composed of objects scattered from a protoplanetary region closer to the Sun than TNOs.

While the global differences (shifts) in colors reflect an evolution which removes or conceals ultrared matter as the objects move closer in the solar system, the large range of colors that KBO, Centaurs and EC shares is likely to result from a common large diversity in 
their composition and not from an evolutionary process. It therefore implies limited mixing in the outer part of the protoplanetary disk leading to the accretion of inhomogeneous planetesimals. This contrasts with the Trojans which exhibit much less diversity, consistent with their formation in a specific region of the disk. Luu and Jewitt (1996) have indeed unsuccessfully attempted to explain the very wide range of colors of the KBOs with an evolutionary mechanism known as "collisional resurfacing". The instantaneous color of the surface of an object is assumed to result from the competition between progressive global reddening caused by cosmic ray bombardment of surface organics and stochastic excavation of less red, unirradiated material due to impacts resulting from mutual collisions between these objects. Numerical simulations indicate that this mechanism is indeed capable of creating significant color changes, but further examination and confrontation with new, more extended measurements led Jewitt and Luu (2001) to conclude that it is not the primary cause of the color diversity among the KBOs. Thébault and Doressoundiram (2003) also concluded from a detailed numerical check that it does not hold in its present simple form.

Processes relevant to Centaurs and comets capable of destroying or concealing the carrier of ultrared matter presumed to be a highly polymerized organic material formed in the transneptunian region (Moroz 2003) have been considered by Jewitt (2002a). The formation of a ballistic mantle by redeposition of debris unable to escape from the body sphere of influence, even if the activity is limited to a small fraction of the total surface area, could result in the global burial of the original KBO type surface. Jewitt (2002a, b) believes that this process is probably ongoing on Chiron and 29P/Schwassmann-Wachmann 1. Delsanti et al. (2004) have incorporated the formation of a ballistic mantle to the basic model of Luu and Jewitt (1996), however in a very simple way. The formation of a rubble mantle by the accumulation on the surface of debris that are too large to be lift off by drag forces could also explain a change in color as this material excavated from some depth will 
have escaped prolonged cosmic ray irradiation and is therefore expected to have different optical properties.

These mechanisms have been proposed in the context of the classical view where, following the rapid formation of a rubble mantle on the cometary surface, its activity is restricted to a few vents, the bulk of the surface $(>90 \%)$ being inactive. This view prevailed after the in-situ observations of the nucleus 1P/Halley (e.g., Keller et al. 1986; Sagdeev et al. 1986) and that of 19P/Borrelly (Soderblom et al. 2001) and is further consistent with their comae being structured by jets. We now have strong arguments against the universality of this scheme.

i) The downward revision of the nucleus sizes coming from our HST and recent ground-based observations (Lamy et al., 2004). Straightforward calculations show that observed production rates require widespread activity on the smallest nuclei and the removal of quite thick layers of material (up to several meters) over the whole surface during each orbital revolution. Consequently new material continuously appears at the surface during the phase of activity of the comet. As examplified by 46P/Wirtanen, the bulk (probably up to 100\%) of the surface of its nucleus becomes active (Lamy et al., 1998a) and a layer of about $0.5 \mathrm{~m}$ is removed at each orbital revolution (Groussin and Lamy, 2003). The formation of a rubble mantle will likely take place as activity decreases but it cannot grow since a thick insulating mantle would quench the activity at the next apparition. This mantle is blown off as the comet comes back and it will indeed be of utmost interest to attempt to detect color variations between these two situations. We are now convinced that there exists a very diverse variety of activity pattern on cometary surface, from very small active areas to widespread activity over their whole surface. In this respect, we recall that we did not find any correlation between color and active fraction (Fig. 9). 
ii) Our observation of ultrared cometary nuclei likely excludes the systematic presence of a redeposited mantle. Here again, this material excavated from some depth will have escaped prolonged cosmic ray irradiation and is therefore expected to lack the ultrared matter.

iii) Mantling should affect all colors in contradiction with our finding of color dependent shifts.

In view of the above difficulties, we favor the scenario where the ultrared matter is progressively removed from ECs, dead comets and some Centaurs as they evolve in the inner solar system, most likely because of its thermal dissociation. For instance thermal dissociation of organic cometary grains has been indirectly observed and modeled (Lamy and Perrin, 1988) and studied in the laboratory (Cottin et al., 2004). A cascade dissociation of unstable complex molecules starting at relatively low temperatures and producing simpler, less red molecules is highly conceivable. Alternatively, thermal transformations between allotropes as seen in sulfur can be invoked. The mechanism of thermal alteration of different organic compounds presents the advantage of differentially affecting the colors as observed, while the rapid burial of ancient surface materials advocated by Jewitt (2002a) cannot and, on the contrary, will affect all colors the same way.

Another potentially interesting mechanism has been mentioned by Grundy and Stansberry (2003), and later elaborated by Grundy (2009) based on the combined optical behaviors of different materials: a transparent, colorless (at visual wavelengths) material such as ice mixed with a red material such as tholin could make its visual reflectance spectrum much more red. The progressive sublimation of ices as the objects travel from the outer to the inner parts of the solar system results in a decrease of the reddening, and in addition of the albedo, a correlation which is now well observed. Here again, models involving burial of ultra red matter by cometary activity as discussed above may have 
difficulty explaining this decline in albedo.

\section{SUMMARY}

In this article, we report color data for 24 active cometary nuclei detected with the Hubble Space Telescope using our well-proven method of nucleus-coma separation. Combining with available (essentially ground-based) data, we built a data base of 44 $(V-R)$ color indices, $15(B-V)$ and $25(R-I)$ color indices for ecliptic comet (EC) nuclei and $7(V-R), 6(B-V)$ and $5(R-I)$ color indices for nearly-isotropic comet nuclei. An extensive statistical analysis of the data set for EC nuclei leads to the following conclusions.

- The colors range from slightly blue to ultra red, invalidating previous reports based on too small data sets. The two bluest nuclei, 14P/Wolf and 19P/Borrelly have however, very large uncertainties affecting the (ground-based) determinations of their $(V-R)$ indices.

- There are no statistically significant correlations between colors, nor between colors and respectively perihelion distance, nucleus size and active fraction; however we note a trend of the colors of the group of red nuclei to slightly increase with increasing perihelion distance.

- Several color indices (but not all) exhibit a trend of decreasing reddening with increasing inclination and dynamical lifetime, two proxies of the age of ECs, but strictly speaking, the Spearman tests remain inconclusive.

In order to perform a comparison of colors of primitive bodies of the solar system, and search for possible genetic relationships and patterns of evolution, we present a very broad compilation of data, distinguishing the following families of objects: classical KBO in low 
(59) and high (52) inclination orbits, Plutinos (49), Scattered Disk Objects (40), Centaurs (32), candidate dead comets (12), and Trojans (85). The above numbers apply to objects having measured $(V-R)$ index, they are slightly less for the two other indices $(B-V)$ and $(R-I)$. We further distinguish two populations of Centaurs on the basis of the bimodality of the distribution of their $(B-R)$ index: Centaur I with $(B-R)>1.7$ and Centaurs II with $(B-R)<1.4$ (there are no objects with $1.4<(B-R)<1.7)$. The analysis of the distribution functions (histograms) and cumulative distribution functions of colors allow to reach the following conclusions.

- CKBO-LI stands as a very distinct family of ultra red objects.

- CKBO-HI, Plutinos and SDO show remarkable similarities of their distributions of $(V-R)$ indices - to the point where one could define a common population - but less so for the other indices.

- The above property holds as well for the global family of Centaurs. The consideration of two groups of Centaurs alters the above picture: Centaur I are ultra red, slightly redder than CKBO-LI while the colors of Centaur II tend to roughly merge with the least reddened objects, Trojans and candidate dead comets.

- ECs, candidate dead comets, and Trojans appear as distinct families.

- Two families, CKBO-LI and ECs lack significant correlations between their color indices, contrary to the other families.

The color differences between the different families primitive objects result from their formation and their evolution at widely different places in the solar system. The observed pattern of colors among primitive bodies is remarkably consistent with the scenario proposed by Gomes (2003) and generalized by the "Nice" model for the TNOs (Levison et al., 2008), and by Morbidelli et al. (2005) for the Trojans. 
- The CKBO-HI, the Plutinos and the SDOs (i.e., the TNO-II using our terminology) formed closer in than the CKBO-LI, the latter being formed in the outer part of the protoplanetary disk. The colors would also support the formation of SDOs at slightly shorter heliocentric distances than the CKBO-HI and the Plutinos, possibly explaining their more efficient scattering.

- Trojans have formed in the trans-neptunian disk, and their past chaotic dynamical evolution has brought them in the inner solar system with perihelia as low as 2 to 3 AU. This naturally explains their deficiency in water and organics (Emery and Brown, 2004), and their homogeneous colors quite similar to those of the candidate dead comets and the Centaurs II.

The bimodal distribution of the colors of Centaurs, ascertained as observations accumulate, could be possibly explained by different evolutionary paths. Centaurs II could represent an evolved population which would have have been scattered back outward, mostly by Jupiter, and consequently have lost their red matter during their residence in the inner solar system. Centaurs I could be composed of TNOs (prominently CKBO-LI) and ultra red objects from a yet unstudied family.

The existence of very red objects among the ECs and the broad diversity of their colors is compatible with an origin in the Kuiper belt and their subsequent evolution in the solar system. The possibility that the classical Kuiper belt could be a source of ECs should be re-examined; interestingly, we noted that CKBOs and ECs share the same properties of low color-color correlations, contrary to the other families.

Candidate dead comets represent almost the ultimate state of evolution of minor bodies in the solar system as they conspicuously appear more evolved than ECs, Trojans and Centaurs II. This likely results from their longer residence in the inner solar system. 
Ultrared matter has been removed from Trojans, Centaurs II and dead comets and is progressively removed from ecliptic comets during their orbital evolution in the inner part of the solar system. Our preferred mechanism is thermal alteration of different organic compounds because it can differentially affect the colors as observed, contrary to the rapid burial of ancient surface materials. Alternatively, a mechanism where colorless ice enhances the redness of organic compounds which then progressively decreases as ice sublimates could work as well.

The very few nuclei of NICs for which we have color measurements ( 7 in the best case) are all slightly redder than the Sun and globally less red than the ECs. This is consistent with their origin in the Oort cloud composed of objects scattered from a region closer to the Sun than TNOs.

As a final remark, we stress that the number of objects for which we have $B V R I$ colors remain small, not to say extremely small, so that the presently available data sets may simply be not representative of the reality. Recent controversies often resulting from biased data sets give the lesson that we must remain prudent in our interpretations.

\section{ACKNOWLEDGEMENTS}

We thank O. Hainaut for access to his data base, A. Doressoundiram and S. Fornasier for providing their color data in tabular form, and A. Delsanti, A. Morbidelli and N. Peixinho for making their articles available to us in advance of publication. Comments from S. Tegler and A. Morbidelli on an early version of this article were very valuable. We thank the Referee H. Levison for his in-depth review and for many valuable comments and suggestions. We acknowledge the support of the French "Programme National de Planétologie", jointly funded by CNRS and CNES, and of the bilateral French-Hungarian cooperation program. I. Toth further acknowledges the support of the Université de Provence, of the Hungarian Academy of Sciences through grant No. 9871, and of the 
Hungarian State Research Foundation for Sciences (OTKA) through grant No. T025049.

\section{REFERENCES}

Abell, P.A., Fernández, Y.R., Pravec, P., French, L.M., Farnham, T.L., Gaffey, M.J., Hardersen, P.S., Kusnirak, P., Sarounova, L., Sheppard, S.S., 2003 Physical characteristics of asteroid-like comet nucleus C/2001 OG108 (LONEOS). LPSC 34, 1253.

A’Hearn, M.F., Millis, R.L., Schleicher, D.G., Osip, D.J., Birch, P.V., 1995. The ensemble properties of comets: Results from narrowband photometry of 85 comets, 1976-1992. Icarus $118,223-270$.

Bailey, B., Malhotra, R., 2008. The Scattered Disk as the source of the Jupiter Family comets. Astron. J. in press.

Barucci, A., Doressoundiram, A., Tholen, D., Fulchignoni, M., Lazzarin, M., 1999. Spectrophotometric observations of Edgeworth-Kuiper Belt Objects. Icarus 142, 476-481.

Barucci, A., Romon, J., Doressoundiram, A., Tholen, D.J., 2000. Compositional surface diversity in the Trans-Neptunian Objects. Astron. J. 120, 496-500.

Bauer, J.M., Fernández, Y.R., Meech, K.J., 2003a. An optical survey of the active centaur C/NEAT (2001 T4). Publ. Astr. Soc. Pacific 115, 981-989.

Bauer, J.M., Meech, K.J., Fernández, Y.R., Pittichova, J., Hainaut, O.R., Boehnhardt, H., Delsanti, A.C., 2003b. Physical survey of 24 Centaurs with visible photometry. Icarus $166,195-211$. 
Benecchi, S.D., Noll, K.S., Grundy, W.M., Buie, M.W., Stephens, D., Levison, H.F., 2008. The colors of transneptunian binaries. Bull. Amer. Astron. Assoc. 40, Abstr. [38.11], p. 466.

Bernstein, G.M., Trilling, D.E., Allen, R.L., Brown, M.E., Holman, M., Malhotra, R., 2004. Astron. J. 128, 1364-1390.

Biretta, J.A., and 18 colleagues, 1996. In: Wide Field and Planetary Camera 2 Instrument Handbook. Version 4.0 (1996). Space Telescope Science Institute, Baltimore.

Boehnhardt H., Rainer N., Birkle K., Schwehm G., 1999. The nuclei of comets 26P/Grigg-Skjellerup and 73P/Schwassmann-Wachmann 3. Astron. Astrophys. 341, 912-917.

Boehnhardt, H., 2001. Visible and near-IR observations of transneptunian objects. Results from ESO and Calar Alto telescopes. Astron. Astrophys. 378, 653-667.

Boehnhardt, H., Delsanti, A., Barucci, A., Hainaut, O., Doressoundiram, A., Lazzarin, M., Barrera, L., de Bergh, C., Birkle, K., Dotto, E., Meech, K.J., Ortiz, J.E., Romon, J., Sekiguchi, T., Thomas, N., Tozzi, G.P., Watanabe, J., West, R.M., 2002. ESO large program on physical studies of Transneptunian Objects and Centaurs: Visible photometry - first results. Astron. Astrophys. 395, 297-303.

Boehnhardt, H., Tozzi, G. P., Bagnulo, S., Muinonen, K., Nathues, A., Kolokolova, L., 2008. Photometry and polarimetry of the nucleus of comet 2P/Encke. Astron. Astrophys. 489, $1337-1343$.

Brown, W.R., Luu, J.X., 1997. CCD photometry of the Centaur 1995 GO. Icarus 126, 218-224.

Brown, M.E., 2001. Inclination distribution of the Kuiper Belt. Astron. J. 121, 2804-2814. 
Brucker, M.J., Grundy, W.M., Stansberry, J.A., Spencer, J.R., Sheppard, S.S., Chiang, E.I., Buie, M.W., 2009. High Albedos of Low Inclination Classical Kuiper Belt Objects. Icarus, in press.

Campins H., A'Hearn M.F., McFadden L-A., 1987. The bare nucleus of comet Neujmin 1. Astrophys. J. 316, 847-857.

Campins, H., Ziffer, J., Licandro, J., Pinilla-Alonso, N., Fernández, Y., De León, J., Mothé-Diniz, Th., Binzel, R.P., 2006. Nuclear spectra of comet 162P/Siding Spring (2004 TU12). Astron. J. 132, 1346-1353.

Carusi, A., Perozzi, E., Valsecchi, G.B., Kresak, L., 1985. Long-Term Evolution of Short-Period Comets. Adam Hilger, Bristol, UK.

Chamberlin A.B., McFadden L-A., Schulz R., Schleicher D.G., Bus S.J., 1996. 4015 Wilson-Harrington, 2201 Oljato, and 3200 Phaethon: Search for CN emission. Icarus $119,173-181$.

Chiang, E., Lithwick, Y., Murray-Clay, R., Buie, M., Grundy, W., Holman, M. 2007. A Brief History of Trans-Neptunian Space. In: Reipurth, B., Jewitt, D., Keil, K. (Eds.), Protostars and Planets V. Univ. of Arizona Press, Tucson, pp. 895-911.

Cottin, H., Bénilan, Y., Gazeau, M.-C., Raulin, F., 2004. Origin of cometary extended sources from degradation of refractory organics on grains: polyoxymethylene as formaldehyde parent molecule. Icarus 167, 397-416.

Cruikshank, D.P., 1977. Radii and albedos of four Trojan asteroids and Jovian satellites 6 and 7. Icarus 30, 224-230.

Cruikshank, D.P., 1987. Dark matter in the solar system. Adv. Space Res. 7 (No. 5), 109-120. 
Davis, D.R., Farinella, P., 1997. Collisional evolution of Edgeworth-Kuiper Belt Objects. Icarus $125,50-60$.

Davis, D.R., Farinella, P., 1998. Evolution of an initial massive Edgeworth-Kuiper Belt: Accretion and collisional history. Bull. Amer. Astr. Soc. 30, p. 1112.

Davies, J.K., McBride, N., Ellison, S.L., Green, S.F., Ballantyne, D.R., 1998. Visible and infrared photometry of six Centaurs. Icarus 134, 213-227.

Davies, J.K., 2000. Physical characteristics of Trans-Neptunian objects and Centaurs. In: Fitzsimmons, A., Jewitt, D., West, R.M. (Eds.), Minor bodies in the Outer Solar System, Springer-Verlag, p. 9-24.

Delahodde C.E., Meech K.J., Hainaut O., Dotto E., 2001. Detailed phase function of comet 28P/Neujmin 1. Astron. Astrophys. 376, 672-685.

Delsanti, A., Hainaut, O., Jourdeuil, E., Meech, K.J., Boehnhardt, H., Barrera, L., 2004. Simultaneous visible-near IR photometry study of Kuiper Belt Object surfaces with the ESO/Very Large Telescopes. Astron. Astrophys. 417, 1145-1158.

Delsanti, A., Peixinho, N., Boehnhardt, H., Barucci, A., Merlin, F., Doressoundiram, A., Davies, J.K., 2006. Near-infrared properties of Kuiper belt objects and centaurs: final results from the ESO large program. Astron J. 131, 1851-1863.

DeMeo, F.E., Fornasier, S., Barucci, M.A., Perna, D., Protopapa, S., Alvarez-Candal, A., Delsanti, A., Doressoundiram, A., Merlin, F., de Bergh, C., 2008. Visible and Near-infrared Colors of TNOs from the Second ESO Large Program. Bull. Amer. Astron. Soc. 40, Abstr. [47.05], p. 482.

Dohnanyi, J.W., 1969. Collisional model of asteroids and their debris. J. Geophys. Res. $74,2531-2554$. 
Dones, L., Levison, H.F., Duncan, M., 1996. On the dynamical lifetimes of planet-crossing objects. In: R.T. Rettig and J.M. Hahn (Eds.), Completing the Inventory of the Solar System, ASP Confer. Ser. Vol. 107, pp. 233-244.

Doressoundiram, A., Barucci, M.A., Romon, J., Veillet, C., 2001. Multicolor photometry of Trans-neptunian Objects. Icarus 154, 277-286.

Doressoundiram, A., Peixinho, N., de Bergh, C., Fornasier, S., Thébault, P., Barucci, M.A., Veillet, C., 2002. The color distribution in the Edgeworth-Kuiper Belt. Astron. J. 124, 2279-2296.

Doressoundiram, A., Peixinho, N., Doucet, C., Mousis, O., Barucci, A.M., Petit, J.M., Veillet, C., 2005. The Meudon Multicolor Survey (2MS) of Centaurs amd trans-neptunian objects: extended dataset and status on the correlations reported. Icarus 174, 90-104.

Doressoundiram, A., Boehnhardt, H., Tegler, S.C., Trujillo, Ch., 2008. Color properties and trends of the transneptunian objects. In: Barucci, A., Boehnhardt, H., Cruikshank, D., Morbidelli, A. (Eds.), The solar system beyond Neptune. University of Arizona Press, Tucson, AZ, pp. 91-104.

Duncan, M., Quinn, T., Tremaine, S., 1988. The origin of short-period comets. Astron. J. 328, L69-L73.

Duncan, M., Levison, H.F., Budd, S.M., 1995. The dynamical structure of the Kuiper Belt. Astron. J. 110, 3073-3081.

Duncan, M., Levison, H.F., 1997. A disk of scattered icy objects and the origin of Jupiter-family comets. Science 276, 1670-1672.

Duncan, M., Levison, H.F., Dones, L., 2004. Dynamical evolution of ecliptic comets. In: Festou, M., Keller, H.U., Weaver, H.A. (Eds.), Comets II. University of Arizona Press, 
Tucson, pp. 193-204.

Durda, D., Stern, S.A., 2000. Collision rates in the present-day Kuiper belt and Centaur regions: Application to surface activation and modification on comets, Kuiper belt Objects, Centaurs, and Pluto-Charon. Icarus 145, 220-229.

Edgeworth, K.E., 1949. The origin and evolution of the Solar System. Mon. Not. R. Astr. Soc. 109, 600-609.

Elliot, J.L., Kern, S.D., Clancy, K.B., Gulbis, A.A.S., Millis, R.L., Buie, M.W., Wasserman, L.H., Chiang, E.I., Jordan, A.B., Trilling, D.E., Meech, K.J., 2005. The Deep Ecliptic Survey: A search for Kuiper belt objects and centaurs. II. Dynamical classification, the Kuiper belt plane, and the core population. Astron. J. 129, 111-1162.

Emel'yanenko, V.V., Asher, D.J., Bailey, M.E., 2003. A new class of trans-Neptunian objects in high-eccentricity orbits. Mon. Not. R. Astr. Soc. 338, 443-451.

Emel'yanenko, V.V., Asher, D.J., Bailey, M.E., 2004. High-eccentricity trans-Neptunian objects as a source of Jupiter-family comets. Mon. Not. R. Astr. Soc. 350, 161-166.

Emery, J., Brown, R.H., 2004. The surface composition of Trojan asteroids: constrains set by scattering theory. Icarus 170, 131-152.

Farinella, P., Davis, D.R., 1996. Short-period comets: primordial bodies or collisional fragments. Science 273, 938-941.

Farinella, P., Davis, D.R., Stern, S.A., 2000. Formation and collisional evolution of the Edgeworth-Kuiper Belt. In: Mannings, V., Boss, A.P., Russell, S.S. (Eds.), Protostars and Planets IV. University of Arizona Press, Tucson, AZ, pp. 1255-1284.

Fernández, J.A., 1980. On the existence of a comet belt beyond Neptune. Mon. Not. R. Astr. Soc. 192, 481-491. 
Fernández, J.A., Ip, W.-H., 1983. On the time evolution of cometary influx in the region of the terrestrial planets. Icarus 54, 377-387.

Fernández, J.A., Ip, W.-H., 1984. Some dynamical aspects of the accretion of Uranus and Neptune - The exchange of orbital angular momentum with planetesimals. Icarus 58, 109-120.

Fernie, J.D., 1983. Relationships between the Johnson and Kron-Cousins VRI photometric systems. Publ. Astr. Soc. Pacific 95, 782-785.

Fitzsimmons, A., Dahlgren, M., Lagerkvist, C.-I., Magnusson, P., Williams, I.P., 1994. A spectroscopic survey of D-type asteroids. Astron. Astrophys. 282, 634-642.

Fleming, H.J., Hamilton, D.P., 2000. On the origin of the Trojan asteroids: effects of Jupiter's mass accretion and radial migration. Icarus 148, 479-493.

Fornasier, S., Dotto, E., Hainaut, O., Marzari, F., Boehnhardt, H., De Luise, F., Barucci, M.A., 2007. Visible spectroscopic and photometric survey of Jupiter Trojans: Final results on dynamical families. Icarus 190, 622-642.

Gladman, B., Kavelaars, J.J., Petit, J-M., Morbidelli, A., Holman, M.J., Loredo, T., 2001. The structure of the Kuiper Belt: Size distribution and radial extent. Astron. J. 122, 1051-1066.

Gladman, B., Holman, M., Grav, T., Kavelaars, J., Nicholson, P., Aksnes, K., Petit, J-M., 2002. Evidence for an extended Scattered Disk. Icarus 157, 269-279.

Gladman, B., Chan, C. 2006. Production of the Extended Scattered Disk by Rogue Planets. Astrophys. J. 643, L135-L138.

Gladman, B., Marsden, B. G., VanLaerhoven, C. 2008. Nomenclature in the Outer Solar System. In: Barucci, M. A., Boehnhardt, H., Cruikshank, D. P., Morbidelli, A. (Eds.), 
The Solar System Beyond Neptune. Univ. of Arizona Press, Tucson, pp. 43-57.

Gomes, R.S., 2003. The origin of the Kuiper Belt high-inclination population. Icarus 161, 404-418.

Gomes, R.S., Morbidelli, A., Levison, H.F., 2004. Planetary migration in a planetesimal disk: why did Neptune stop at 30 AU ? Icarus 170, 492-507.

Gomes, R.S., Fernández, J.A., Gallardo, T., Brunini, A., 2008. The scattered disk: origins, dynamics and end states. In: Barucci, A., Boehnhardt, H., Cruikshank, D., Morbidelli, A. (Eds.), Kuiper Belt. University of Arizona Press, Tucson. In press.

Gradie, J., Tedesco, E., 1982. Compositional structure of the asteroid belt. Science 216, 1405-1409.

Gradie, J.C., Chapman, C.R., Tedesco, E.F., 1989. Distribution of taxonomic classes and the compositional structure of the asteroid belt. In: Binzel, R.P., Gehrels, T., Matthews, M.S. (Eds.), Asteroids II. Univ. of Arizona Press, Tucson, pp. 316-335.

Green, S.F., McBride, N., O’Ceallaigh, D.P., Fitzsimmons, A., Williams, I.P., Irwin, M.J., 1997. Surface reflectance properties of distant solar system objects. Mon. Not. R. Astr. Soc. $290,186-192$.

Groussin, O., Lamy, P.L., 2003. Activity on the surface of the nucleus of comet 46P/Wirtanen. Astron. Astrophys. 412, 879-891.

Grundy, W.M., 2009. Is the Missing Ultra-Red Material Colorless Ice? Icarus, in press. E-print: astro-ph - arXiv:0811.2433.

Grundy, W.M., Noll, K.S., Stephens, D.C., 2005. Diverse Albedos of Small Trans-Neptunian Objects. Icarus 176, 184-191. 
Gulbis, A.A.S., Elliot, J.L., Kane, J.F., 2006. The color of the Kuiper belt core. Icarus 183, 168-178.

Hahn, J.M., Malhotra, R., 1999. Orbital evolution of planets embedded in a planetesimal disk. Astron. J. 117, 3041-3053.

Hainaut, O., Delsanti, A.C., 2002. Colors of Minor Bodies in the Outer Solar System. A statistical analysis. Astron. Astrophys. 389, 641-664.

Holtzman, J.A., Burrows, Ch.J., Casertano, S., Hester, J.J., Trauger, J.T., Watson, A.M., Worthy, G., 1995. The photometry performance and calibration of WFPC2. Publ. Astr. Soc. Pacific 107, 1065-1093.

Horner, J., Evans, N.W., Bailey, M.E., 2004a. Simulations of the populations of Centaurs I. The bulk statistics. Mon. Not. R. Astr. Soc. 354, 798-810.

Horner, J., Evans, N.W., Bailey, M.E., 2004b. Simulations of the populations of Centaurs II. Individual objects. Mon. Not. R. Astr. Soc. 355, 321-329.

Ip, W.-H., 1989. Dynamical processes of macro-accretion of Uranus and Neptune - A first look. Icarus 80, 167-178.

Ip, W.-H., Fernández, J.A., 1991. Steady-state injection of short-period comets from the trans-Neptunian cometary belt. Icarus 92, 185-193.

Jewitt, D.C., 1996. From comets to asteroids: When hairy stars go bald. Earth, Moon, Planets 72, 185-201.

Jewitt, D.C., 1999. Kuiper Belt Objects. Annu. Rev. Earth. Planet. Sci. 27, 287-312.

Jewitt, D.C., 2002a. From Kuiper Belt Objects to cometary nucleus: The missing ultrared matter. Astron. J. 123, 1039-1049. 
Jewitt, D.C., 2002b. From Kuiper belt object to cometary nucleus. In: Wrmbein, B. (Ed.), Proc. of Asteroids, Comets, Meteors VIIIth, 2002, Berlin. ESA SP-500, Ed. B. Wrmbein, ESA Publ. Division, ESTEC, Noordwijk, The Netherlands, pp. 11-19.

Jewitt, D., 2005. A first look at the Damocloids. Astron. J., 129, 530-538.

Jewitt D.C., Meech K.J., 1988. Optical properties of cometary nuclei and a preliminary comparison with asteroids. Astrophys. J. 328, 974-986.

Jewitt D.C., Luu J., 1989. A CCD portrait of comet P/Tempel 2. Astron. J. 97, 1766-1790, Plates Nos. 105-106, pp. 1984-1842.

Jewitt, D.C., Luu, J.X., 1990. CCD spectra of asteroids. II - The Trojans as spectral analogs of cometary nuclei. Astron. J. 100, 933-944.

Jewitt, D.C., Kalas, P., 1998. Thermal observations of Centaur 1997 CU26. Astrophys. J. 499, L103-L106.

Jewitt, D.C., Luu, J.X., 1998. Optical-infrared spectral diversity in the Kuiper Belt. Astron. J. 115, 1667-1670.

Jewitt, D.C., Luu, J.X., Trujillo, C., 1998. Large Kuiper belt objects. The Mauna Kea 8k CCD survey. Astron. J. 115, 2125-2135.

Jewitt, D.C., Luu, J.X., 2000. Physical nature of the Kuiper Belt. In: Mannings, V., Boss, A.P., Russell, S.S. (Eds.), Protostars and Planets IV. Univ. Arizona Press, Tucson, AZ, p. $1201-1230$.

Jewitt, D.C., Trujillo, C.A., Luu, J.X., 2000. Population and size distribution of small Jovian Trojan asteroids. Astron, J. 120, 1140-1147.

Jewitt, D.C., Luu, J.X., 2001. Colors and spectra of Kuiper Belt Objects. Astron. J. 122, 2099-2114. 
Jewitt D., Sheppard S., Fernández Y.R., 2003. 143P/Kowal-Mrkos and the shapes of cometary nuclei. Astron. J. 125, 3366-3377.

Jewitt, D. Peixinho, N., Hsieh, H.H., 2007. U-Band Photometry of Kuiper Belt Objects. Astron. J. 134, 2046-2053.

Kary, D.M., Lissauer, J.J., 1995. Nebular gas drag and planetary accretion. II. Planet on an eccentric orbit. Icarus 117, 1-24.

Keller, H.U., and 17 colleagues, 1986. First Halley Multicolour Camera imaging results from GIOTTO. Nature 321, 320-326.

Krist, J., 1995. Simulation of HST PSF using Tiny TIM. In: R.A. Shaw, H.E. Payne, J.J.E. Hayes (Eds.), ASP Conference Series Vol. 77 - Astronomical Data Analysis Software and System IV. pp. 349-352.

Kuchner, M.J., Brown, M.E., Holman, M., 2002. Long-term dynamics and the orbital inclinations of the classical Kuiper Belt Objects. Astron. J. 124, 1221-1230.

Lamy, P.L., Perrin, J.-M., 1988. Optical properties of organic grains - Implications for interplanetary and cometary dust. Icarus 76, 100-109.

Lamy, P.L., Toth, I., Jorda, L., Weaver, H.A., 1998a. The nucleus and the inner coma of comet 46P/Wirtanen. Astron. Astrophys. 335, L25-L29.

Lamy, P.L., Toth, I., Weaver, H.A., 1998b. Hubble Space Telescope Observations of the nucleus and inner coma of comet 19P/Borrelly 1994l. Astron. Astrophys. 337, 945-954.

Lamy, P.L., Toth, I., A’Hearn, M.F., Weaver, H.A., 1999. Hubble Space Telescope observations of the nucleus of comet 45P/Honda-Mrkos-Pajdusakova and its inner coma. Icarus $140,424-438$. 
Lamy, P.L., Toth, I., Jorda, L., Groussin, O., A'Hearn, M.F., Weaver, H.A., 2002. The nucleus of comet 22P/Kopff and its inner coma. Icarus 156, 442-455.

Lamy, P.L., Toth, I., Fernández, Y.R., Weaver, H.A., 2004. The sizes, shapes, albedos, and colors of cometary nuclei. In: Festou, M., Keller, H.U., Weaver, H.A. (Eds.), Comets II. University of Arizona Press, Tucson, AZ, pp. 223-264.

Lamy, P.L., Toth, I., Weaver, H.A., Jorda, L., Kaasalainen, M., Gutiérrez, P.J., 2006. Hubble Space Telescope observations of the nucleus and inner coma of comet 67P/Churyumov-Gerasimenko. Astron. Astrophys., 458, 669-678.

Lamy, P.L., Toth, I., Weaver, H.A., A'Hearn, M.F., Jorda, L., 2008. Properties of the nucleus and coma of 13 ecliptic comets observed with the Hubble Space Telescope. Astron. Astrophys., submitted.

Lamy, P.L., Toth, I., Weaver, H.A., 2007. Hubble Space Telescope observations of the nucleus of comet 29P/Schwassmann-Wachmann 1 and its inner coma. Icarus, in preparation.

Lamy, P.L., Toth, I., Weaver, H.A., A'Hearn, M.F., Jorda, L., 2009. Properties of the nucleus and coma of 13 ecliptic comets from Hubble Space Telescope snapshot observations. Astron. Asdtrophys., submitted.

Lawrence, K.K., Helin, E.F., Pravdo, S., 2001. Comet C/2001 M10 (NEAT). IAU Circ. 7654 .

Levison, H.F., and Duncan, M.J., 1994. Long-term dynamical behavior of short-period comets. Icarus 108, 18-36.

Levison, H.F., 1996. Comet taxonomy. In: Rettig, T.W., Hahn, J.M. (Eds.), Completing the Inventory of the Solar System. ASP Confer. Ser. Vol. 107, pp. 173-192. 
Levison, H.F., Duncan, M.J., 1997. From the Kuiper Belt to Jupiter-Family comets: The spatial distribution of ecliptic comets. Icarus 127, 13-32.

Levison, H.F., Shoemaker, E.M., Shoemaker, C.S., 1997. Dynamical evolution of Jupiter's Trojan asteroids. Nature 385, 42-44.

Levison, H.F., Dones, L., Duncan, M., 2001. The origin of Halley-type comets: probing in the Oort cloud. Astron. J. 121, 2253-2267.

Levison, H.F., Stern, S.A., 2001. On the size dependence of the inclination distribution of the Main Kuiper Belt. Astron, J., 121, 1730-1735.

Levison, H.F., Morbidelli, A., 2003. The formation of the Kuiper belt by the outward transport of bodies during Neptune's migration. Nature 426, 419-421.

Levison, H.F., Morbidelli, A., Dones, L., 2004. Sculpting the Kuiper belt by a stellar encounter: Constraints from the Oort cloud and Scattered Disk. Astron. J. 128, 2553-2563.

Levison, H.F., Morbidelli, A., Vanlaerhoven, Ch., Gomes, R., Tsiganis, L., 2008. Origin of the Structure of the Kuiper Belt during a Dynamical Instability in the Orbits of Uranus and Neptune. Icarus 196, 258-273.

Lewis, J. S., 1972. Low temperature condensation from the solar nebula. Icarus 16, 241-252.

Li, J.-Y., A’Hearn, M.F., Belton, M.J.S., Crockett, C.J., Farnham, T.L., Lisse, C.M., McFadden, L.A., Meech, K.J., Sunshine, J.M., Thomas, P.C., Veverka, J., 2007. Deep Impact photometry of comet $9 \mathrm{P} /$ Tempel 1 . Icarus, submitted.

Licandro, J., Tancredi G., Lindgren M., Rickman H., Gil Hutton R., 2000. CCD photometry of cometary nuclei, I: Observations from 1990-1995. Icarus 147, 161-179. 
Licandro, J., Guerra, J.C., Campins, H., Di Martino, M., Lara, L.M., Gil-Hutton, R., Tozzi, G.P., 2002. The surface of cometary nuclei related minor bodies. Earth, Moon, and Planets 90, 495-496.

Licandro, J., Campins, H., del Leon Cruz, J., Gil-Hutton, R., Lara-Lopez, L.M., 2003. Near infrared spectroscopy TNOs, Centaurs and comet nuclei. Bull. Amer. Astr. Soc. 35, Abstr. [39.11]

Lowry S.C., Weissman P.R., 2003. CCD observations of distant comets from Palomar and Steward observatories. Icarus 164, 492-503.

Lowry, S.C., Fitzsimmons, A., Collander-Brown, S., 2003. CCD photometry of distant comets. II. Ensemble properties of Jupiter-family comets. Astron. Astrophys. 397, 329-343.

Lowry, S.C., Weissman, P.R., 2007. Rotation and color properties of the nucleus of Comet 2P/Encke. Icarus 188, 212-223.

Luu, J.X., Jewitt, D.C., 1990a. Cometary activity of 2060 Chiron. Astron. J. 100, 913-932.

Luu, J.X., Jewitt, D.C., 1990b. Charge-coupled device spectra of asteroids. I - Near-Earth and 3:1 resonance asteroids. Astron. J. 99, 1985-2011.

Luu, J.X., Jewitt, D.C., 1990c. The nucleus of comet P/Encke. Icarus 86, 69-81.

Luu, J.X., 1993. Spectral diversity among the nuclei of comets. Icarus 104, 138-148.

Luu, J.X., Jewitt, D.C., 1996. Color diversity among the Centaurs and Kuiper Belt Objects. Astron. J. 112, 2310-2318.

Luu, J., Marsden, B.G., Jewitt, D., Trujillo, C.A., Hergenrother, C.W., Chen, J., Offutt, W.B., 1997. A new dynamical class in the Trans-Neptunian solar system. Nature 387, $573-575$. 
Luu, J.X., Jewitt, D.C., 2002. Kuiper Belt Objects: Relics from the accretion disk of the Sun. Annu. Rev. Astron. Astrophys. 40, 63-101.

Malhotra, R., 1993. Orbital resonances in the solar nebula - Strengths and weaknesses. Icarus 106, 264-27.

Malhotra, R., 1995. The origin of the Pluto's orbit: Implications for the solar system beyond Neptune. Astron. J. 110, 420-429.

Malhotra, R., 1998. Pluto's inclination excitation by resonance sweeping. LPSC 29, Abstr. No. 1476 .

Malhotra, R., Duncan, M.L., Levison, H.F., 2000. Dynamics of the Kuiper Belt. In: Mannings, V., Boss, A.P., Russell, S.S. (Eds.), Protostars and Planets IV. University of Arizona Press, Tucson, AZ, pp. 1231-1254.

Marzari, F., Farinella, P., Vanzani, V. 1995. Are Trojan collisional families a source for short-period comets? Astron. Astrophys. 299, 267-276.

Marzari, F., Farinella, P., Davis, D.R., Scholl, H., Campo Bagatin, A., 1997. Collisional evolution of Trojan asteroids. Icarus 125, 39-49.

Marzari, F., Scholl, H., 1998. The growth of Jupiter and Saturn and capture of Jupiter Trojans. Astron. Astrophys. 339, 278-285.

McBride, N., Davies, J.K., Green, S.F., Foster, M.J., 1999. Optical and infrared observations of the Centaur 1997 CU26. Mon. Not. Roy. Astr. Soc. 306, 799-805.

Meech, K.J., Hainaut, O., Marsden, B.G., 2004. Comet nucleus size distribution from HST and Keck Telescopes. Icarus, in press.

Meech, K.J., Hainaut, O., Marsden, B.G., 2004. Comet nucleus size distribution from HST and Keck Telescopes. Icarus 170, 463-491. 
Millis R.L., A'Hearn M.F., Campins H., 1988. An investigation of the nucleus and coma of comet P/Arend-Rigaux. Astrophys. J., 324, 1194-1209.

Morbidelli, A., 1997. An overview of Kuiper Belt and on the origin of Jupiter-family comets. Cel. Mech. Dyn. Astr. 56, 129-156.

Morbidelli, A., 1998. The structure of the Kuiper Belt and the origin of Jupiter-family comets. In: Solar System formation and Evolution. ASP Conf. Ser. 149, 83-105.

Morbidelli, A., Brown, M.E., Levison, H.F., 2003. The Kuiper Belt and its primordial sculpting. Earth, Moon, and Planets 92, 1-27.

Morbidelli, A., Brown, M.E., 2004. Structure and dynamical evolution of the population of TNOs. In: Festou, M., Keller, H.U., Weaver, H.A. (Eds.), Comets II. Univ. of Arizona Press, Tucson, AZ, 175-191.

Morbidelli, A., Emel'yanenko, V., Levison, H.F., 2004. Origin and orbital distribution of the Trans-Neptunian scattered disk. Mon. Not. R. Soc. 355, 935-940.

Morbidelli, A., Levison, H.F., Tsiganis, K., Gomes, R., 2005. Chaotic capture of Jupiter's Trojan asteroids in the early solar system. Nature 435, 462-465.

Morbidelli, A., Levison, H.F., Gomes, R., 2008. The dynamical structure of the Kuiper belt and its primordial origin. In: Barucci, A., Boehnhardt, H., Cruikshank, D., Morbidelli, A. (Eds.), Kuiper Belt. University of Arizona Press, Tucson, AZ. In press.

Morbidelli, A., Levison, H.F., Gomes, R., 2008. The dynamical structure of the Kuiper belt and its primordial origin. In: Barucci, A., Boehnhardt, H., Cruikshank, D., Morbidelli, A. (Eds.), The solar system beyond Neptune. University of Arizona Press, Tucson, AZ, pp. 275-292. 
Moroz, L.V., Baratta, G., Distefano, E., Strazzulla, G., Starukhina, L.V., Dotto, E., Barucci, M.A., 2003. Ion irradiation of asphaltite: Optical effects and implications for Trans-Neptunian Objects and Centaurs. Earth, Moon, and Planets 92, 279-289.

Mueller, B.E.A., Hergenrother, C.W., Samarasinha, N.H., Campins, H., McCarthy, D.W. Jr., 2003. Visible and IR observations of the outer solar system object 29981 (1999 TD10). Bull. Amer. Astr. Soc. 35, Abstr. [39.13].

Nagasawa, M., Tanaka, H., Ida, S., 2000. Orbital evolution of asteroids during depletion of the solar nebula. Astron. J., 119, 1480-1497.

Noll, K.S., Grundy, W.M., Stephens, D.C., Levison, H.F., Kern, S.D., 2008. Evidence for two populations of classical transneptunian objects: The strong inclination dependence of classical binaries. Icarus 194, 758-768.

Peale, S.J., 1993. The effect of the solar nebula on the Trojan precursors. Icarus 106, 308-322.

Peixinho, N., Doressoundiram, A., Delsanti, A., Boehnhardt, H., Barucci, M.A., Belskaya, I., 2003. Reopening the TNOs color controversy: Centaurs bimodality and TNOs unimodality. Astron. Astrophys. 410, L29-L32.

Peixinho, N., Boehnhardt, H., Belskaya, I., Doressoundiram, A., Barucci, M.A., Delsanti, A.C., 2004. ESO large Program on Centaurs and TNOs: Visible colors - Final results. Icarus 170, 153-166.

Peixinho, N., 2008. Deconstructing the correlation among Classical KBOs: In the end there can be only one. Bull. Amer. Astron. Soc. 40, Abstr. [38.08], p. 465.

Peixinho, N., Lacerda, P. Jewitt, D., 2008. Color-Inclination Relation of the Classical Kuiper Belt Objects. Astron. J. 136, 1837-1845. 
Petit, J-M., Morbidelli, A., Valsecchi, G.B., 1999. Large scattered planetesimals and the excitation of the small body population. Icarus 141, 367-387.

Press, W.H., Teukolsky, S.A., Vetterling, W.T., Flannery, B.P., 1992. Numerical Recipes in FORTRAN. The art of scientific computing. 2nd edition, Cambridge Univ. Press, New York.

Rickman, H., Froeschlé, Ch., Froeschlé, Cl., Valsecchi, G.B., 2004. Stellar perturbations on the scattered disk. Astron. Astrophys. 428, 673-681.

Romanishin, W., Tegler, S. C., Consolmagno, G., 2008. Colors of Inner Disk KBOs. Asteroids, Comets, Meteors X. Baltimore, MD. LPI Contribution No. 1405, paper I.D. 8331.

Sagdeev, R.Z., and 37 colleagues, 1986. Television observations of comet Halley from VEGA spacecraft. Nature 321, 262-266.

Schaefer, B.E., Rabinowitz, D¿L., Tourellotte, S.W., 2008. Division of icy bodies into groups based on surface properties Bull. Amer. Astr. Soc. 40, Abstr. [47.04], p. 482.

Schulz, R., 2002. Trans-Neptunian Objects. Astron. Astrophys. Rev. 11, 1-31.

Shoemaker, E.M., Shoemaker, C., Wolfe, R.F., 1989. Trojan asteroids - Populations, dynamical structure and origin ot the L4 amd L5 swarms. In: Binzel, R.P., T. Gehrels, T., Matthews, M.S. (Eds.), Asteroids II. University of Arizona Press, Tucson, pp. 487-523.

Snodgrass, C., Fitzsimmons, A., Lowry, S.C., 2005. The nuclei of comets 7P/Pons-Winnecke, 14P/Wolf and 92P/Sanguin. Astron. Astrophys. 444, 287-295.

Snodgrass, C., Lowry, S.C., Fitzsimmons, A., 2006. Photometry of cometary nuclei: rotation rates, colours and a comparison with Kuiper belt objects. Mon. Not. R. Astron. 
Soc. $373,1590-1602$.

Snodgrass, C., Lowry, S.C., Fitzsimmons, A., 2008. Optical observations of 23 distant Jupiter Family Comets, including 36P/Whipple at multiple phase angles. Mon. Not. R. Astron. Soc. 385, 737-756.

Soderblom, L.A., Becker, T.L., Bennett, G., and 19 colleagues, 2002. Observations of comet 19P/Borrelly by the Miniature Integrated Camera and Spectrometer aboard Deep Space 1. Science 296, 1087-1091.

Stansberry, J. A., Grundy, W. M., Brown, M., Cruikshank, D. P., Spencer, J., Trilling, D., Margot, J.-L., 2008. Physical Properties of Kuiper Belt Objects and Centaurs: Spitzer Space Telescope Constraints. In: Barucci, M. A., Boehnhardt, H., Cruikshank, D. P., Morbidelli, A. (Eds.), The Solar System Beyond Neptune. Univ. of Arizona Press, Tucson, pp. 161-179.

Stephens, D.C., Noll, K.S., Grundy, W.M., Millis, R.L., Spencer, J., Buie, M. Tegler, S.C., Romanishin, W., Cruikshank, D.P., 2003. HST photometry of trans-Neptunian Objects. Earth, Moon, and Planetes 92, 251-260.

Stern, S.A., 1995. Collisional time scales in the Kuiper Disk and their implications. Astron, J. 110, 856-868.

Stern, S.A., Colwell, J.E., 1997. Accretion of Edgeworth-Kuiper Belt: forming 100-1000 km bodies at $30 \mathrm{AU}$ and beyond. Astron. J. 114, 841-849.

Stern, S.A., 2003. The evolution of comets in the Oort cloud and Kuiper belt. Nature 424, 639-642.

Sunshine, J. M., A’Hearn, M. F., Groussin, O., Li, J.-Y., Belton, M. J. S., Delamere, W. A., Kissel, J., Klaasen, K. P., McFadden, L. A., Meech, K. J., Melosh, H. J., Schultz, P. 
H., Thomas, P. C., Veverka, J., Yeomans, D. K., Busko, I. C., Desnoyer, M., Farnham, T. L., Feaga, L. M., Hampton, D. L., Lindler, D. J., Lisse, C. M., Wellnitz, D. D., 2006. Exposed water ice deposits on the surface of comet 9P/Tempel 1. Science 311, 1453-1455.

Tegler, S.C., Romanishin, W. 1998. Two distinct populations of Kuiper-Belt Objects. Nature 392, 49-51.

Tegler, S.C., Romanishin, W., 2000. Extremely red Kuiper-belt objects in near-circular orbits beyond 40 AU. Nature 407, 979-981.

Tegler, S.C., Romanishin, W., 2003. Resolution of the Kuiper belt object color controversy: two distinct color populations. Icarus 161, 181-191.

Tegler, S.C., Bauer, J.M., Romanishin, W., Peixinho, N., 2008. Colors of centaurs. In: Barucci, A., Boehnhardt, H., Cruikshank, D., Morbidelli, A. (Eds.), The solar system beyond Neptune. University of Arizona Press, Tucson, AZ, pp. 105-114.

Thébault, P., Doressoundiram, A., 2003. Colors and collision rates within the Kuiper belt: problems with the collisional resurfacing scenario. Icarus 162, 27-37.

Thommes, E.W., Duncan, M.J., Levison, H.F., 1999. The formation of Uranus and Neptune in the Jupiter-Saturn region of the solar system. Nature 402, 635-638.

Thomas, N., Keller, H.U., 1989. The colour of comet P/Halley's nucleus and dust. Astron. Astrophys. 213, 487-494.

Tiscareno, M.S., Malhotra, R., 2003. The dynamics of known Centaurs. Astron. J. 126, 3122-3131.

Torbett, M.V., 1989. Chaotic motion in a comet disk beyond Neptune. The delivery of short-period comets. Astron, J. 98, 1477-1481. 
Toth, I., Lamy, P., Weaver, H., A’Hearn, M., Kaasalainen, M., Lowry, S., 2006. HST observations of the nucleus fragment 73P/Schwassmann-Wachmann 3-C during its close approach to Earth in 2006. Bull. Amer. Astr. Soc. 38, Abstr. [06.01], p. 1063.

Trujillo, C.A., Brown, M.E., 2002. A correlation between inclination and color in the Kuiper Belt. Astrophys. J. 566, L125-L128.

Trujillo, C.A., Brown, M.E., 2003. The Caltech Wide Area Sky Survey. Earth, Moon, and Planets 92, 99-112.

Tubiana, C., Barrera, L., Drahus, M., Boehnhardt, H., 2008. Comet 67P/ChuryumovGerasimenko at a large heliocentric distance. Astron. Astrophys. 490, 377-386.

Yu, Q., Tremaine, S., 1999. The dynamics of Plutinos. Astron, J. 118, 1873-1881. 
Table 1

Characteristics of the filters

\begin{tabular}{ccc}
\hline Name & $\bar{\lambda}^{a}$ & $\Delta \lambda^{b}$ \\
\hline F336W & 334 & 38 \\
F439W & 423 & 47 \\
F555W & 540 & 123 \\
F675W & 670 & 89 \\
F814W & 792 & 149 \\
\hline
\end{tabular}

${ }^{a}$ Mean wavelength (nm).

${ }^{b}$ Equivalent width (nm).

Definitions are given in Biretta et al. (1996). 
Table 2

Journal of observations

\begin{tabular}{|c|c|c|c|c|c|c|c|}
\hline Comet & Date $^{a}$ & Orbits $^{b}$ & Filters $^{c}$ & $\mathrm{r}_{\mathrm{h}}^{d}$ & $\Delta^{e}$ & $\alpha^{f}$ & Scale $^{g}$ \\
\hline 4P/Faye & 2000 Feb 07.618 & 1 & $V, R$ & $2.956^{O}$ & 1.998 & 5.58 & 66.0 \\
\hline $8 \mathrm{P} /$ Tuttle & 2007 Dec 10.560 & 12 & $B, V, R, I$ & $1.25^{I}$ & 0.48 & 46.6 & 16.0 \\
\hline 10P/Tempel 2 & 1999 Jun 23.432 & 1 & $B, V, R, I$ & $1.669^{I}$ & 0.681 & 12.75 & 22.5 \\
\hline 17P/Holmes & 1999 Jun 15.527 & 1 & $V, R$ & $3.116^{I}$ & 2.176 & 8.46 & 71.8 \\
\hline 22P/Kopff & 1996 Jul 18.732 & 8 & $B, V, R, I$ & $1.588^{O}$ & 0.574 & 4.29 & 18.9 \\
\hline $37 \mathrm{P} /$ Forbes & 1999 Oct 29.326 & 1 & $V, R, I$ & $2.270^{\mathrm{O}}$ & 1.389 & 14.84 & 45.8 \\
\hline 44P/Reinmuth 2 & 2000 Jun 12.638 & 1 & $V, R$ & $2.728^{I}$ & 1.732 & 5.29 & 57.2 \\
\hline $45 \mathrm{P} / \mathrm{HMP}^{*}$ & 1996 Feb 04.773 & 2 & $U, B, V, R, I$ & $0.982^{\mathrm{O}}$ & 0.169 & 93.21 & 5.6 \\
\hline 46P/Wirtanen & 1996 Aug 28.660 & 8 & $V, R$ & $2.447^{I}$ & 1.507 & 10.93 & 49.7 \\
\hline 47P/Ashbrook-Jackson & 2000 Jul 26.541 & 8 & $B, V, R$ & $2.571^{I}$ & 1.588 & 7.16 & 52.4 \\
\hline $50 \mathrm{P} /$ Arend & 2000 Jan 11.854 & 1 & $V, R, I$ & $2.374^{\mathrm{O}}$ & 1.468 & 11.77 & 48.4 \\
\hline 55P/Tempel-Tuttle & 1998 Jan 09.697 & 4 & $B, V, R, I$ & $1.260^{I}$ & 0.462 & 44.32 & 15.3 \\
\hline 59P/Kearns-Kwee & 2000 Jan 15.542 & 1 & $V, R, I$ & $2.516^{O}$ & 1.539 & 3.26 & 50.8 \\
\hline 63P/Wild 1 & 2000 Apr 22.212 & 1 & $V, R, I$ & $2.266^{\mathrm{O}}$ & 1.299 & 9.16 & 42.9 \\
\hline $67 \mathrm{P} / \mathrm{CG}^{* *}$ & 2003 Mar 11.873 & 11 & $V, R$ & $2.506^{O}$ & 1.526 & 4.79 & 50.4 \\
\hline 70P/Kojima & 2001 Mar 29.446 & 8 & $V, R$ & $2.517^{O}$ & 1.529 & 4.29 & 50.5 \\
\hline 71P/Clark & 2000 Mar 12.701 & 1 & $V, R$ & $2.715^{I}$ & 1.764 & 7.51 & 58.2 \\
\hline 84P/Giclas & 2000 Jan 13.444 & 1 & $V, R$ & $2.209^{\mathrm{O}}$ & 1.368 & 16.86 & 45.2 \\
\hline 86P/Wild 3 & 2001 May 26.257 & 8 & $B, V, R$ & $2.316^{I}$ & 1.304 & 1.81 & 43.0 \\
\hline 106P/Schuster & 1999 Oct 18.708 & 1 & $B, V, R, I$ & $1.666^{I}$ & 0.780 & 23.00 & 25.8 \\
\hline 110P/Hartley 3 & 2000 Nov 24.704 & 8 & $V, R$ & $2.578^{I}$ & 1.607 & 5.06 & 53.0 \\
\hline $112 \mathrm{P} /$ Urata-Niijima & 1999 Sep 08.618 & 1 & $V, R$ & $2.296^{I}$ & 1.501 & 19.15 & 49.5 \\
\hline 114P/Wiseman-Skiff & 2000 Jan 14.444 & 1 & $B, V, R, I$ & $1.569^{\mathrm{O}}$ & 0.836 & 33.22 & 27.6 \\
\hline \multirow[t]{7}{*}{ C/Hale-Bopp (1995 O1) } & 1995 Oct 23.27 & 1 & $B, V, R$ & $6.360^{I}$ & 6.714 & 8.16 & 221.3 \\
\hline & 1996 Apr 07.50 & 1 & $B, V, R$ & $4.795^{I}$ & 4.812 & 11.97 & 158.7 \\
\hline & 1996 May 20.45 & 1 & $B, V, R$ & $4.360^{I}$ & 3.690 & 10.87 & 121.7 \\
\hline & 1996 Jun 22.49 & 1 & $B, V, R$ & $4.015^{I}$ & 3.037 & 4.45 & 100.2 \\
\hline & 1996 Jul 25.60 & 1 & $B, V, R$ & $3.659^{I}$ & 2.746 & 8.24 & 90.4 \\
\hline & 1996 Sep 23.18 & 1 & $B, V, R$ & $2.981^{I}$ & 2.945 & 19.49 & 97.1 \\
\hline & 1996 Oct 17.63 & 1 & $B, V, R$ & $2.697^{I}$ & 3.040 & 18.76 & 100.2 \\
\hline
\end{tabular}

Notes:

${ }^{a}$ Mid date of the observational session bracketed by the first and last images (UT).

${ }^{b}$ Number of HST orbits devoted to the observations.

${ }^{c} U, B, V, R, I=$ filters F336W,F439W,F555W,F675W,F814W.

${ }^{d}$ Heliocentric distance (AU), either $I$ : Inbound (pre-perihelion) or $O$ : outbound (post-perihelion).

${ }^{e}$ Geocentric distance (AU).

$f$ Solar phase angle $\left({ }^{\circ}\right)$.

$g$ Pixel scale at the nucleus distance $(\mathrm{km})$.

* 45P/Honda-Mrkos-Pajdusakova

** 67P/Churyumov-Gerasimenko 
Table 3

Colors of cometary nuclei observed with the HST

\begin{tabular}{|c|c|c|c|c|c|c|c|c|c|c|}
\hline Comet & Class $^{a}$ & $T_{J}^{b}$ & $P_{\text {orb }}{ }^{c}$ & $q^{d}$ & $Q^{e}$ & $e^{f}$ & $i^{g}$ & $(B-V)$ & $(V-R)$ & $(R-I)$ \\
\hline 4P/Faye & $\mathrm{EC}$ & 2.76 & 7.5 & 1.66 & 6.02 & 57 & 9.0 & & $5 \pm 0.04$ & - \\
\hline $8 \mathrm{P} /$ Tuttle & $\mathrm{NIC}$ & 1.58 & 13.6 & 1.03 & 10.37 & 0.82 & 55.0 & $0.89 \pm 0.04$ & $0.53 \pm 0.04$ & $0.53 \pm 0.06$ \\
\hline 10P/Tempel 2 & $\mathrm{EC}$ & 2.97 & 5.5 & 1.48 & 4.73 & 0.52 & 12.0 & $0.80 \pm 0.02$ & $0.49 \pm 0.03$ & $0.52 \pm 0.03$ \\
\hline 17P/Holmes & $\mathrm{EC}$ & 2.87 & 7.1 & 2.17 & 5.20 & 0.41 & 19.2 & & $0.56 \pm 0.02$ & - \\
\hline 22P/Kopff & $\mathrm{EC}$ & 2.76 & 7.3 & 1.59 & 5.96 & 0.58 & 9.1 & $0.82 \pm 0.05$ & $0.50 \pm 0.05$ & $0.50 \pm 0.05$ \\
\hline $37 \mathrm{P} /$ Forbes & $\mathrm{EC}$ & 2.87 & 6.1 & 1.45 & 5.25 & 0.57 & 7.2 & & $0.29 \pm 0.03$ & $0.66 \pm 0.06$ \\
\hline 44P/Reinmuth 2 & $\mathrm{EC}$ & 2.93 & 6.6 & 1.89 & 5.17 & 0.46 & 7.0 & & $0.62 \pm 0.08$ & - \\
\hline $45 \mathrm{P} / \mathrm{HMP}^{*}$ & $\mathrm{EC}$ & 2.58 & 5.3 & 0.53 & 5.52 & 0.82 & 4.2 & $1.08 \pm 0.05$ & $0.44 \pm 0.05$ & $0.21 \pm 0.05$ \\
\hline 46P/Wirtanen & $\mathrm{EC}$ & 2.82 & 5.5 & 1.06 & 5.13 & 0.66 & 11.7 & - & $0.45 \pm 0.06$ & - \\
\hline 47P/Ashbrook-Jackson & $\mathrm{EC}$ & 2.91 & 7.5 & 2.31 & 5.33 & 0.40 & 12.5 & $0.78 \pm 0.08$ & $0.40 \pm 0.08$ & - \\
\hline $50 \mathrm{P} /$ Arend & $\mathrm{EC}$ & 2.70 & 8.2 & 1.92 & 6.25 & 0.53 & 19.2 & - & $0.81 \pm 0.10$ & $0.26 \pm 0.09$ \\
\hline 55P/Tempel-Tuttle & $\mathrm{NIC}$ & -0.63 & 33.2 & 0.98 & 19.70 & 0.91 & 162.5 & $0.75 \pm 0.05$ & \pm 0.05 & $0.42 \pm 0.05$ \\
\hline 59P/Kearns-Kwee & $\mathrm{EC}$ & 2.78 & 9.4 & 2.34 & 6.60 & 0.48 & 9.4 & - & $0.62 \pm 0.07$ & $0.27 \pm 0.08$ \\
\hline 63P/Wild 1 & $\mathrm{EC}$ & 2.42 & 13.2 & 1.96 & 9.23 & 0.65 & 19.9 & - & $0.50 \pm 0.05$ & $0.42 \pm 0.04$ \\
\hline $67 \mathrm{P} / \mathrm{CG}^{* *}$ & $\mathrm{EC}$ & 2.75 & 6.6 & 1.29 & 5.72 & 0.63 & 7.12 & - & $0.51 \pm 0.06$ & - \\
\hline 70P/Kojim & $\mathrm{EC}$ & 2.91 & 7.0 & 2.00 & 5. & 0.45 & 6.6 & - & & - \\
\hline 71P/Clark & $\mathrm{EC}$ & 2.99 & 5.5 & 1.56 & 4.68 & 0.50 & 9.5 & - & $0.64 \pm 0.07$ & - \\
\hline 84P/Giclas & $\mathrm{EC}$ & 2.88 & 7.0 & 1.85 & 5.44 & 0.49 & 7.3 & - & $0.32 \pm 0.03$ & - \\
\hline 86P/Wild 3 & $\mathrm{EC}$ & 2.94 & 6.9 & 2.31 & 4.96 & 0.36 & 15.4 & $1.58 \pm 0.08$ & $0.86 \pm 0.10$ & - \\
\hline 106P/Schuster & $\mathrm{EC}$ & 2.69 & 7.3 & 1.55 & 5.97 & 0.59 & 20.1 & $1.01 \pm 0.06$ & $0.52 \pm 0.06$ & $0.45 \pm 0.06$ \\
\hline 110P/Hartley 3 & $\mathrm{EC}$ & 3.00 & 6.9 & 2.48 & 4.75 & 0.31 & 11.7 & - & $0.67 \pm 0.09$ & - \\
\hline 112P/Urata-Niiji & EC & 2.70 & 6.7 & 1.46 & 5.62 & 0.59 & 24.2 & - & $0.53 \pm 0.04$ & - \\
\hline 114P/Wiseman-Skiff & $\mathrm{EC}$ & 2.78 & 6.7 & 1.57 & 5.51 & 0.56 & 18.3 & $0.85 \pm 0.03$ & $0.46 \pm 0.02$ & $0.54 \pm 0.02$ \\
\hline C/Hale-Bopp (1995 O1) & NIC & 0.06 & 2549.1 & 0.91 & 372.29 & 0.9951 & 89.4 & $0.81 \pm 0.05$ & $0.39 \pm 0.05$ & - \\
\hline
\end{tabular}

${ }^{a}$ Orbital family class (EC: Ecliptic Comet, NIC: Nearly-Isotropic Comet).

$b$ Tisserand parameter with respect to the Jupiter.

$c$ Orbital period (year).

$d$ Perihelion distance (AU).

${ }^{e}$ Aphelion distance (AU).

$f$ Eccentricity.

${ }^{g}$ Inclination $\left(^{\circ}\right)$.

* 45P/Honda-Mrkos-Pajdusakova

** 67P/Churyumov-Gerasimenko 
Table 4

Colors of cometary nuclei from other observations

\begin{tabular}{|c|c|c|c|c|c|}
\hline Comet & $(B-V)$ & $(V-R)$ & $(R-I)$ & Phot. $^{a}$ & Ref. $^{b}$ \\
\hline 1P/Halley & $0.72 \pm 0.04$ & $0.41 \pm 0.03$ & $0.39 \pm 0.06$ & $\mathrm{~F} / \mathrm{HMC}$ & TK89 \\
\hline \multirow{6}{*}{$2 \mathrm{P} /$ Encke } & $0.78 \pm 0.02$ & $0.48 \pm 0.02$ & - & $\mathrm{S}$ & LJ90 \\
\hline & - & $0.43 \pm 0.05$ & - & $\mathrm{F}$ & J02 \\
\hline & - & $0.38 \pm 0.06$ & - & $\mathrm{F}$ & J02 \\
\hline & - & $0.37 \pm 0.09$ & - & $\mathrm{F}$ & HD02 \\
\hline & $0.73 \pm 0.06$ & $0.39 \pm 0.06$ & - & $\mathrm{F}$ & $\mathrm{Lo}+07$ \\
\hline & - & $0.47 \pm 0.07$ & - & $\mathrm{F}$ & $\mathrm{B}+08$ \\
\hline \multirow[t]{3}{*}{$6 \mathrm{P} / \mathrm{d}^{\prime}$ Arrest } & $0.78 \pm 0.04$ & $0.54 \pm 0.04$ & $0.45 \pm 0.04$ & $\mathrm{~F}$ & J02 \\
\hline & - & $0.62 \pm 0.08$ & - & $\mathrm{F}$ & $\mathrm{M}+04$ \\
\hline & $1.08 \pm 0.12$ & $0.33 \pm 0.09$ & $0.33 \pm 0.12$ & $\mathrm{~F}$ & LW03 \\
\hline 7P/Pons-Winnecke & - & $0.40 \pm 0.05$ & $0.41 \pm 0.06$ & $\mathrm{~F}$ & $\mathrm{~S}+05$ \\
\hline 9P/Tempel 1 & $0.84 \pm 0.01$ & $0.50 \pm 0.01$ & $0.49 \pm 0.02$ & $\mathrm{~S}$ & $\mathrm{Li}+07$ \\
\hline \multirow[t]{3}{*}{ 10P/Tempel 2} & - & $0.53 \pm 0.03$ & - & $\mathrm{F}$ & JM88 \\
\hline & - & $0.58 \pm 0.03$ & - & $\mathrm{S}$ & JL89 \\
\hline & - & $0.56 \pm 0.02$ & - & $\mathrm{F}$ & $\mathrm{M}+04$ \\
\hline $14 \mathrm{P} /$ Wolf & - & $0.57 \pm 0.07$ & $0.51 \pm 0.06$ & $\mathrm{~F}$ & $\mathrm{~S}+05$ \\
\hline 17P/Holmes & - & $0.41 \pm 0.07$ & $0.44 \pm 0.08$ & $\mathrm{~F}$ & $\mathrm{~S}+06$ \\
\hline 19P/Borrelly & - & $0.25 \pm 0.78$ & - & $\mathrm{F}$ & $\mathrm{Lo}+03$ \\
\hline 21P/Giacobini-Zinner & $0.80 \pm 0.03$ & $0.50 \pm 0.02$ & - & $\mathrm{S}$ & L93 \\
\hline 26P/Grigg-Skjellerup & - & $0.30 \pm 0.10$ & - & $\mathrm{F}$ & $\mathrm{B}+99$ \\
\hline \multirow[t]{5}{*}{ 28P/Neujmin 1} & - & $0.46 \pm 0.04$ & - & $\mathrm{F}$ & $\mathrm{Ca}+87$ \\
\hline & - & $0.45 \pm 0.05$ & - & $\mathrm{F}$ & JM88 \\
\hline & - & $0.50 \pm 0.04$ & - & $\mathrm{F}$ & JM88 \\
\hline & - & $0.45 \pm 0.05$ & - & $\mathrm{F}$ & $\mathrm{D}+01$ \\
\hline & - & $0.48 \pm 0.06$ & - & $\mathrm{F}$ & $\mathrm{M}+04$ \\
\hline 36P/Whipple & - & $0.48 \pm 0.03$ & $0.62 \pm 0.02$ & $\mathrm{~F}$ & $\mathrm{~S}+08$ \\
\hline \multirow[t]{2}{*}{ 47P/Ashbrook-Jackson } & & $0.40 \pm 0.30$ & - & $\mathrm{F}$ & $\mathrm{Lic}+00$ \\
\hline & & $0.45 \pm 0.03$ & $0.38 \pm 0.03$ & $\mathrm{~F}$ & $\mathrm{~S}+06$ \\
\hline 48P/Johnson & - & $0.50 \pm 0.30$ & - & $\mathrm{F}$ & $\mathrm{Lic}+00$ \\
\hline \multirow{4}{*}{ 49P/Arend-Rigaux } & $.77 \pm 0.03$ & $0.47 \pm 0.01$ & $0.43 \pm 0.02$ & $\mathrm{~F}$ & $\mathrm{M}+88$ \\
\hline & - & $0.47 \pm 0.01$ & - & $\mathrm{S}$ & L93 \\
\hline & - & $0.40 \pm 0.30$ & - & $\mathrm{F}$ & $\mathrm{Lic}+00$ \\
\hline & - & $0.49 \pm 0.11$ & $0.54 \pm 0.14$ & $\mathrm{~F}$ & $\mathrm{Lo}+03$ \\
\hline 53P/Van Biesbroeck & - & $0.34 \pm 0.08$ & - & $\mathrm{F}$ & $\mathrm{M}+04$ \\
\hline 67P/Churyumov-Gerasimenko & - & $0.54 \pm 0.03$ & $0.47 \pm 0.03$ & $\mathrm{~F}$ & $\mathrm{Tu}+08$ \\
\hline \multirow[t]{2}{*}{$73 \mathrm{P} / \mathrm{SW} 3$} & - & $0.32 \pm 0.17$ & - & $\mathrm{F}$ & $\mathrm{B}+99$ \\
\hline & $1.16 \pm 0.20$ & $0.57 \pm 0.11$ & - & $\mathrm{F}$ & $\mathrm{T}+06$ \\
\hline 86P/Wild 3 & - & $0.12 \pm 0.14$ & - & $\mathrm{F}$ & $\mathrm{M}+04$ \\
\hline 92P/Sanguin & - & $0.54 \pm 0.04$ & $0.54 \pm 0.04$ & $\mathrm{~F}$ & $\mathrm{~S}+05$ \\
\hline 94P/Russell 4 & - & $0.62 \pm 0.05$ & $0.44 \pm 0.06$ & $\mathrm{~F}$ & $\mathrm{~S}+08$ \\
\hline \multirow{2}{*}{ 96P/Machholz 1} & - & $0.43 \pm 0.03$ & - & $\mathrm{F}$ & $\mathrm{M}+04$ \\
\hline & - & $0.30 \pm 0.05$ & - & $\mathrm{F}$ & $\mathrm{Lic}+00$ \\
\hline \multirow[t]{4}{*}{ 107P/Wilson-Harrington } & - & $0.31 \pm 0.03$ & - & $\mathrm{S}$ & $\mathrm{Ch}+96$ \\
\hline & - & $0.41 \pm 0.02$ & - & $\mathrm{F}$ & $\mathrm{M}+04$ \\
\hline & $0.61 \pm 0.05$ & $0.20 \pm 0.04$ & - & $\mathrm{F}$ & LW03 \\
\hline & $0.75 \pm 0.06$ & - & - & $\mathrm{F}$ & LW03 \\
\hline 121P/Shoemaker-Holt 2 & - & $0.53 \pm 0.03$ & $0.44 \pm 0.03$ & $\mathrm{~F}$ & $\mathrm{~S}+08$ \\
\hline 131P/Mueller 2 & - & $0.45 \pm 0.12$ & - & $\mathrm{F}$ & $\mathrm{S}+08$ \\
\hline 137P/Shoemaker-Levy 2 & - & $0.71 \pm 0.18$ & $0.54 \pm 0.15$ & $\mathrm{~F}$ & $\mathrm{~S}+06$ \\
\hline \multirow[t]{3}{*}{ 143P/Kowal-Mrkos } & $0.84 \pm 0.02$ & $0.58 \pm 0.02$ & $0.55 \pm 0.02$ & $\mathrm{~F}$ & $\mathrm{~J}+03$ \\
\hline & $0.80 \pm 0.02$ & $0.58 \pm 0.02$ & $0.57 \pm 0.02$ & $\mathrm{~F}$ & $\mathrm{~J}+03$ \\
\hline & - & $0.46 \pm 0.08$ & - & $\mathrm{F}$ & LF05 \\
\hline 162P/Siding Spring & $0.76 \pm 0.01$ & $0.45 \pm 0.01$ & $0.45 \pm 0.03$ & $\mathrm{~S}$ & $\mathrm{Ca}+06$ \\
\hline P/Larsen (2004 H2) & - & $0.49 \pm 0.03$ & $0.28 \pm 0.04$ & $\mathrm{~F}$ & $\mathrm{~S}+08$ \\
\hline P/Siding Spring (2006 HR30) & $0.82 \pm 0.02$ & $0.45 \pm 0.01$ & $0.50 \pm 0.02$ & $\mathrm{~F}$ & HB07 \\
\hline C/2001 OG108 (LONEOS) & $0.76 \pm 0.03$ & $0.46 \pm 0.02$ & $0.44 \pm 0.03$ & $\mathrm{~F}$ & $\mathrm{~A}+03$ \\
\hline
\end{tabular}


${ }^{a} \mathrm{~F}=$ filter photometry, $\mathrm{S}=$ spectrophotometry; HMC = GIOTTO Halley Multicolour Camera

${ }^{b}$ References: A+03 (Abell et al. 2003); B+99 (Boehnhardt et al. 1999);

$\mathrm{Ca}+87, \mathrm{Ca}+06$ (Campins et al. 1987, 2006); Ch+96 (Chamberlin et al. 1996); D+01 (Delahodde et al. 2001); HB07 (Hicks $\&$ B Bauer 2007); HD02 (Hainaut \& Delsanti 2002); JM88 (Jewitt \& Meech 1988);

JL89 (Jewitt \& Luu 1989); Li+07 (Li et al. 2007); J+03 (Jewitt et al. 2003); LJ90 (Luu Es Jewitt 1990c);

L93 (Luu 1993); Lic+00 (Licandro et al. 2000); Lo+03 (Lowry et al. 2003); LF05 (Lowry \&5 Ftizsimmons 2005); LW03 (Lowry \& Weissman 2003); M+88 (Millis et al. 1988); M+04 (Meech et al. 2004);

$\mathrm{S}+05, \mathrm{~S}+06, \mathrm{~S}+08$ (Snodgrass et al. 2005, 2006, 2008); T+06 (Toth et al. 2006); TK (Thomas E Keller 1989); $\mathrm{Tu}+08$ (Tubiana et al. 2008). 
Table 5

Adopted colors of cometary nuclei listed in Table 4

\begin{tabular}{|c|c|c|c|c|c|c|c|c|c|c|}
\hline Comet & Class & $T_{J}$ & Porb & $q$ & $Q$ & e & $i$ & $(B-V)$ & $(V-R)$ & $(R-I)$ \\
\hline Solar colors & & & & & & & & 0.0 & 0.35 & 0.28 \\
\hline 1P/Halley & $\mathrm{IIC}$ & -0.60 & 76.0 & 0.59 & 35.3 & 0.97 & 162.2 & $.72 \pm 0$ & $0.41 \pm$ & $0.39 \pm 0.06$ \\
\hline 2P/Encke & EC & 3.03 & & 0.34 & 4.1 & 0.85 & & rit & & \\
\hline $6 \mathrm{P} / \mathrm{d}^{\prime}$ Arrest & $\mathrm{EC}$ & 2.72 & 6.5 & 1.35 & 5.63 & 0.61 & 19.5 & $.00 \pm$ & $0.01 \pm$ & $0.42 \pm 0.09$ \\
\hline 7P/Pons-Winnecke & EC & 2.67 & 6.4 & 1.26 & 5.62 & 0.63 & 22.3 & $U=$ & 0.49 & $0.52 \pm 0.03$ \\
\hline 9P/Tempel 1 & EC & 2.97 & 5.5 & 1.51 & 4.74 & 0.57 & 10.5 & $84 \pm 0$ & $0.50 \pm 0.01$ & $0.49 \pm 0.02$ \\
\hline 10P/Tempel 2 & $\mathrm{EC}$ & 2.97 & 5.4 & 1.46 & 4.73 & 0.53 & 12.1 & $.80 \pm 0.02$ & $0.54 \pm 0.03$ & $0.52 \pm 0.03$ \\
\hline $14 \mathrm{P}^{\prime} /$ Wolf & EC & 2.73 & 8.2 & 2.41 & 5.73 & 0.41 & 27.5 & - & $0.57 \pm 0.07$ & $0.51 \pm 0.06$ \\
\hline 17P/Holmes & $\mathrm{EC}$ & 2.87 & 7.1 & 2.17 & 5.20 & 0.41 & 19.2 & - & $0.53 \pm 0.07$ & $0.44 \pm 0.08$ \\
\hline 19P/Borrelly & $\mathrm{EC}$ & 2.58 & 6.9 & 1.36 & 5.86 & 0.62 & 30.3 & - & $0.25 \pm 0.78$ & - \\
\hline 21P/Giacobini-Zinner & EC & 2.48 & 6.6 & 1.03 & 6.01 & 0.71 & 31.9 & $.80 \pm 0.03$ & $0.50 \pm 0.02$ & - \\
\hline 26P/Grigg-Skjellerup & EC & 2.82 & 5.1 & 1.00 & 4.93 & 0.66 & 21.1 & - & $0.30 \pm 0.10$ & - \\
\hline 28P/Neujmin 1 & $\mathrm{EC}$ & 2.17 & 18.2 & 1.55 & 12.29 & 0.78 & 14.2 & - & $0.47 \pm 0.05$ & - \\
\hline 36P/Whipple & EC & 2.95 & $8.5:$ & 3.09 & 5.24 & 0.25 & 9.9 & - & $0.47 \pm 0.02$ & $0.60 \pm 0.02$ \\
\hline 47P/Ashbrook-Jackson & EC & 2.91 & 7.5 & 2.31 & 5.33 & 0.40 & 12.5 & $.78 \pm 0.08$ & $30.43=$ & $0.38 \pm 0.03$ \\
\hline 48P/Johnson & EC & 2.95 & 7.0 & 2.31 & 4.99 & 0.37 & 13.7 & - & $0.50 \pm 0.30$ & 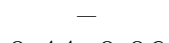 \\
\hline 49P/Arend-Rigav & $\mathrm{EC}$ & 2.72 & 6.6 & 1.37 & 5.68 & 0.61 & 18.3 & $.77 \pm 0.03$ & $0.47 \pm 0.05$ & $0.44 \pm 0.06$ \\
\hline 53P/Van Biesbroeck & EC & 2.66 & 12.5 & 2.42 & 8.36 & 0.55 & 6.6 & - & $0.34 \pm 0.08$ & - \\
\hline 67P/Churyumov-Ger & $\mathrm{EC}$ & 2.75 & 6.6 & 1.29 & 5.72 & 0.63 & 7.12 & - & $0.53 \pm 0.04$ & $0.47 \pm 0.03$ \\
\hline 73P/SW3* & EC & 2.79 & 5.3 & 0.93 & 5.18 & 0.69 & 11.4 & $.16 \pm 0.20$ & $0.45 \pm 0.20$ & - \\
\hline 92P/Sanguin & $\mathrm{EC}$ & 2.41 & 12.4 & 1.81 & 8.92 & 0.66 & 18.8 & - & $0.54 \pm 0.04$ & $0.54 \pm 0.04$ \\
\hline 94P/Rus & $\mathrm{EC}$ & $\sim 3.0$ & 6 & 2.24 & 4.79 & 0.36 & 6. & - & $0.62 \pm 0.05$ & $0.44 \pm 0.06$ \\
\hline 96P/Machholz 1 & NIC & 1.95 & 5.2 & 0.12 & 5.90 & 0.96 & 60.2 & - & $0.38 \pm 0.07$ & - \\
\hline 107P/Wilson-Har & EC & 3.09 & 4.3 & 1.00 & 4.29 & 0.62 & 2.8 & $.67 \pm 0.09$ & $0.33 \pm 0.07$ & 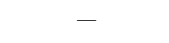 \\
\hline 121P/Shoemaker-Holt 2 & $\mathrm{Fo}$ & 2.88 & 8.0 & 2.65 & 5.36 & 0.34 & 17.7 & - & $0.53 \pm 0.03$ & $0.44 \pm 0.03$ \\
\hline 131P/Mueller 2 & EC & 2.98 & 7.1 & 2.42 & 4.95 & 0.34 & 7.3 & - & $0.45 \pm 0.12$ & - \\
\hline 137P/Shoemaker-Levy 2 & EC & 2.67 & 9.4 & 1.87 & 7.01 & 0.58 & 4.7 & - & $0.71 \pm 0.18$ & $0.54 \pm 0.15$ \\
\hline 143P/Kowal-Mrkos & $\mathrm{EC}$ & 2.87 & 8.9 : & 2.55 & 6.07( & 0.41 & 4.7 & $2 \pm 0$ & $0.58 \pm 0.02$ & $0.56 \pm 0.02$ \\
\hline 162P/Siding Spring & EC & 2.80 & 5.3 & 1.23 & 4.87( & 0.60 & 27.8 & $.76 \pm 0.01$ & 0.45 & $0.45 \pm 0.03$ \\
\hline P/Larsen (2004 H2) & EC & 2.82 & 9.6 & 2.61 & 6.38 & 0.42 & 11.8 & - & $0.49 \pm$ & $0.28 \pm 0.04$ \\
\hline $\mathrm{P} /$ Siding Sprinধ & NIC & 1.77 & 21.9 & 1.23 & 14.41 & 0.84 & 31.9 & $2 \pm 0$ & $0.45 \pm 0.0$ & $0.50 \pm 0.02$ \\
\hline C/LONEOS (2001 OG108 & NIC & 0.62 & 48.5 & 0.99 & 25.61 & 0.93 & 80.2 & $.76 \pm 0.0$ & $0.46 \pm 0.02$ & $0.44 \pm 0.03$ \\
\hline
\end{tabular}

See Table 3 for notations.

*73P/SW3: 73P/Schwassmann-Wachmann 3. 
Table 6

The distribution of colors of cometary nuclei of ecliptic comets in two different size ranges

\begin{tabular}{lcccc}
\hline Color & $\begin{array}{c}\text { All EC } \\
\text { Mean } \pm \text { variance }\end{array}$ & $\begin{array}{c}\text { EC } r_{\text {eff }}<2 \mathrm{~km} \\
\text { Mean } \pm \text { variance }\end{array}$ & $\begin{array}{c}\text { EC } r_{\text {eff }} \geq 2 \mathrm{~km} \\
\text { Mean } \pm \text { variance }\end{array}$ & Weighted* $^{*}$ \\
\hline$(B-V)$ & $0.89 \pm 0.06$ & $0.96 \pm 0.10$ & $0.80 \pm 0.01$ & $\mathrm{~N}$ \\
& $0.86 \pm 0.06$ & $0.94 \pm 0.10$ & $0.81 \pm 0.03$ & $\mathrm{Y}$ \\
$(V-R)$ & $0.51 \pm 0.02$ & $0.52 \pm 0.03$ & $0.49 \pm 0.03$ & $\mathrm{~N}$ \\
& $0.49 \pm 0.07$ & $0.48 \pm 0.06$ & $0.51 \pm 0.08$ & $\mathrm{Y}$ \\
$(R-I)$ & $0.45 \pm 0.03$ & $0.42 \pm 0.04$ & $0.48 \pm 0.02$ & $\mathrm{~N}$ \\
& $0.46 \pm 0.05$ & $0.43 \pm 0.07$ & $0.49 \pm 0.05$ & $\mathrm{Y}$ \\
\hline
\end{tabular}

* The last column indicates whether the uncertainties on the measurements were introduced as weights in the calculation of the means and variances.

Table 7

The number of primitive objects having known color indices

\begin{tabular}{lcccc}
\hline \multicolumn{1}{c}{ Family } & Total & $(B-V)$ & $(V-R)$ & $(R-I)$ \\
\hline ALL KBOs & 160 & 143 & 158 & 117 \\
CKBO-HI & 52 & 44 & 51 & 34 \\
CKBO-LI & 59 & 54 & 59 & 44 \\
Plutinos & 49 & 45 & 48 & 38 \\
SDOs & 40 & 37 & 38 & 28 \\
Centaurs & 32 & 29 & 32 & 26 \\
ECs & 44 & 15 & 44 & 25 \\
Dead comets & 12 & 12 & 12 & 12 \\
Trojans & 85 & 79 & 85 & 85 \\
3:1 reson. asteroids & 23 & 23 & 23 & 23 \\
\hline
\end{tabular}

Table 8

Spearman rank correlation $\rho$ and significance level SL for color-color relationships

\begin{tabular}{lcccccccccc}
\hline Family & $\begin{array}{c}(V-R) \\
\rho\end{array}$ & vs. $(B-V)$ & $(R-I)$ & vs. $(V-R)$ & $(R-I)$ & vs. $(B-V)$ & $(R-I)$ & vs. $(B-R)$ & $(V-I)$ vs. $(B-V)$ \\
& & & $(\mathrm{SL} \%)$ & $\rho$ & $(\mathrm{SL} \%)$ & $\rho$ & $(\mathrm{SL} \%)$ & $\rho$ & $(\mathrm{SL} \%)$ \\
\hline CKBO-LI & -0.01 & $(5)$ & -0.11 & $(45)$ & 0.03 & $(12)$ & 0.07 & $(26)$ & -0.07 & $(29)$ \\
CKBO-HI & 0.53 & $(100)$ & 0.43 & $(99)$ & 0.53 & $(100)$ & 0.56 & $(100)$ & 0.55 & $(100)$ \\
Plutinos & 0.75 & $(100)$ & 0.54 & $(100)$ & 0.75 & $(100)$ & 0.78 & $(100)$ & 0.81 & $(100)$ \\
SDOs & 0.74 & $(100)$ & 0.41 & $(97)$ & 0.65 & $(100)$ & 0.54 & $(100)$ & 0.76 & $(100)$ \\
Centaurs & 0.78 & $(100)$ & 0.71 & $(100)$ & 0.87 & $(100)$ & 0.85 & $(100)$ & 0.87 & $(100)$ \\
ECs & 0.57 & $(98)$ & 0.06 & $(22)$ & -0.08 & $(19)$ & 0.09 & $(21)$ & 0.15 & $(34)$ \\
NICs & 0.31 & $(46)$ & 0.60 & $(72)$ & 1.00 & $(100)$ & -1.00 & $(100)$ & 0.90 & $(96)$ \\
\hline
\end{tabular}


Table 9

One-dimensional and two dimensional Kolmogorov-Smirnov probabilities (\%) for relationships based on $(B-V),(V-R)$ and $(R-I)$ color indices

\begin{tabular}{|c|c|c|c|c|c|c|}
\hline Related families & $(B-V)$ & $(V-R)$ & $(R-I)$ & $\begin{array}{c}(B-V) \\
\& \\
(V-R)\end{array}$ & $\begin{array}{c}(V-R) \\
\& \\
(R-I)\end{array}$ & $\begin{array}{c}(B-V) \\
\& \\
(R-I)\end{array}$ \\
\hline CKBO-LI - Centaur & 0 & 0 & 0 & 0 & 0 & 1 \\
\hline CKBO-LI - EC & 0 & 0 & 0 & 0 & 0 & 0 \\
\hline CKBO-HI - Centaur & 4 & 27 & 4 & 6 & 28 & 7 \\
\hline CKBO-HI - EC & 1 & 0 & 1 & 1 & 1 & 2 \\
\hline Plutino - Centaur & 30 & 78 & 30 & 56 & 44 & 23 \\
\hline Plutino - EC & 13 & 1 & 13 & 1 & 3 & 2 \\
\hline SDO - Centaur & 15 & 84 & 15 & 27 & 67 & 13 \\
\hline $\mathrm{SDO}-\mathrm{EC}$ & 29 & 4 & 29 & 3 & 13 & 6 \\
\hline Centaur - EC & 8 & 7 & 8 & 3 & 4 & 1 \\
\hline EC - dead comet & 1 & 1 & & 1 & 0 & 1 \\
\hline
\end{tabular}

Table 10

One-dimensional and two dimensional Kolmogorov-Smirnov probabilities (\%) for relationships based on $(B-V),(V-R)$ and $(R-I)$ color indices considering two groups of Centaurs

\begin{tabular}{lcccccc}
\hline \multicolumn{1}{c}{ Related objects } & $(B-V)$ & $(V-R)$ & $(R-I)$ & $(B-V)$ & $(V-R)$ & $(B-V)$ \\
& & & & $\begin{array}{c}\text { \& } \\
(V-R)\end{array}$ & $(R-I)$ & $(R-I)$ \\
& & & & & $(R-I)$ \\
\hline CKBO-LI - Centaur I & 2 & 44 & 2 & 1 & 1 & 1 \\
CKBO-LI - Centaur II & 0 & 0 & 0 & 0 & 0 & 0 \\
CKBO-HI - Centaur I & 0 & 0 & 0 & 0 & 0 & 0 \\
CKBO-HI - Centaur II & 0 & 0 & 6 & 0 & 2 & 0 \\
Plutino - Centaur I & 0 & 0 & 1 & 0 & 0 & 0 \\
Plutino - Centaur II & 0 & 1 & 2 & 1 & 2 & 0 \\
SDO - Centaur I & 0 & 0 & 0 & 0 & 0 & 0 \\
SDO - Centaur II & 1 & 2 & 60 & 1 & 9 & 3 \\
Centaur I - EC & 0 & 0 & 0 & 0 & 0 & 0 \\
Centaur II - EC & 0 & 37 & 8 & 1 & 8 & 1 \\
\hline
\end{tabular}

Notes:

Centaurs I: $(B-V)>0.9$

Centaurs II: $(B-V)<0.9$ 


\section{FIGURE CAPTIONS}

Fig. 1a: One HST image and a typical fit of the azimuthally averaged radial profiles for comets 4P/Faye, 10P/Tempel 2, 17P/Holmes, 37P/Forbes, 44P/Reinmuth 2, and 47P/Ashbrook-Jackson. For each comet, the top figure displays the radial profiles where the thick solid line represents the observation, the dashed line is the estimated signal from the nucleus, the thin solid line is the estimated coma, and the dash-dot line is the fitted model; the bottom figure displays the residuals in percents.

Fig. 1b: Same as Fig. 1a for comets 50P/Arend, 59P/Kearns-Kwee, 63P/Wild 1, 70P/Kojima, 71P/Clarck, and 84P/Giclas.

Fig. 1c: Same as Fig. 1a for comets 86P/Wild 3, 106P/Schuster, 110P/Hartley 3, 112P/UrataNiijima, 114P/Wiseman-Skiff, and 8P/Tuttle.

Fig. 2a: Reflectivity spectra of the nuclei of ecliptic comets (circles) and nearly isotropic comets (squares). Our HST observations are represented by filled symbols while the other observations (mostly ground-based) are represented by open symbols. When not shown, error bars are smaller than the size of the symbols. The spectra, normalized at $550 \mathrm{~nm}$, are shifted for clarity.

Fig. 2b: See Fig. 2a

Fig. 3: Distribution of the color indices $(B-V),(V-R),(R-I)$ and $(B-R)$ of cometary nuclei.

Fig. 4: Color-color diagrams for the cometary nuclei. The "reddening lines" correspond to constant reflectivity gradients ranging from -10 to $+50 \% / 100 \mathrm{~nm}$, the tick marks being separated by $10 \% / 100 \mathrm{~nm}$.

Fig. 5: The four color indices $(B-V),(V-R),(R-I)$ and $(B-R)$ of cometary nuclei (from top to bottom) versus their perihelion distance.

Fig. 6: The four color indices $(B-V),(V-R),(R-I)$ and $(B-R)$ of cometary nuclei (from top to bottom) versus their orbital inclination. 
Fig. 7: The four color indices $(B-V),(V-R),(R-I)$ and $(B-R)$ of cometary nuclei (from top to bottom) versus the dynamical lifetime parameter $d_{J}$.

Fig. 8: The four color indices $(B-V),(V-R),(R-I)$ and $(B-R)$ of cometary nuclei (from top to bottom) versus their effective radius.

Fig. 9: The $(V-R)$ color index of the cometary nuclei versus their active fraction.

Fig. 10: Distributions of the color indices for the different families of primitive bodies of the solar system. Columns from left to right: $(B-V),(V-R),(R-I)$, and $(B-R)$. Rows from top to bottom: CKBO-LI, CKBO-HI, Plutinos, SDOs, Centaurs, EC, Candidate dead comets, and Trojans. The means of the distributions with their standard deviations are indicated.

Fig. 11: Color-color diagrams for the different families of primitive bodies. See Fig. 4 for a description of the "reddening lines".

Fig. 12: The $(V-R)$ color index of different families of primitive bodies versus their semimajor axis.

Fig. 13: Cumulative distribution functions of the $(B-V),(V-R),(R-I)$ and $(B-R)$ color indices for the different families of primitive bodies.

Fig. 14: Same as Fig. 13 except that i) the family of TNO II regroups CKBO-HI, Plutinos and SDOs and ii) the family of Centaurs is split into two groups according to their $(B-R)$ index.

Fig. 15a: Individual comparison of the cumulative distribution functions of the $(V-R)$ color index of two families. The left panels display the original functions. In the central panels, the functions of the bluest families are shifted so as to maximize the Kolmogorov-Smirnov probability shown in the right panels.

Fig. 15b: See Fig. 15a

Fig. 16: Summary of the relative color differences between the families of primitive bodies: $(B-V)$ (top), $(V-R)$ (middle) and $(R-I)$ (bottom). The differences were calculated 
using two estimators, the means of the distributions and the Kolmogorov-Smirnov probabilities. 

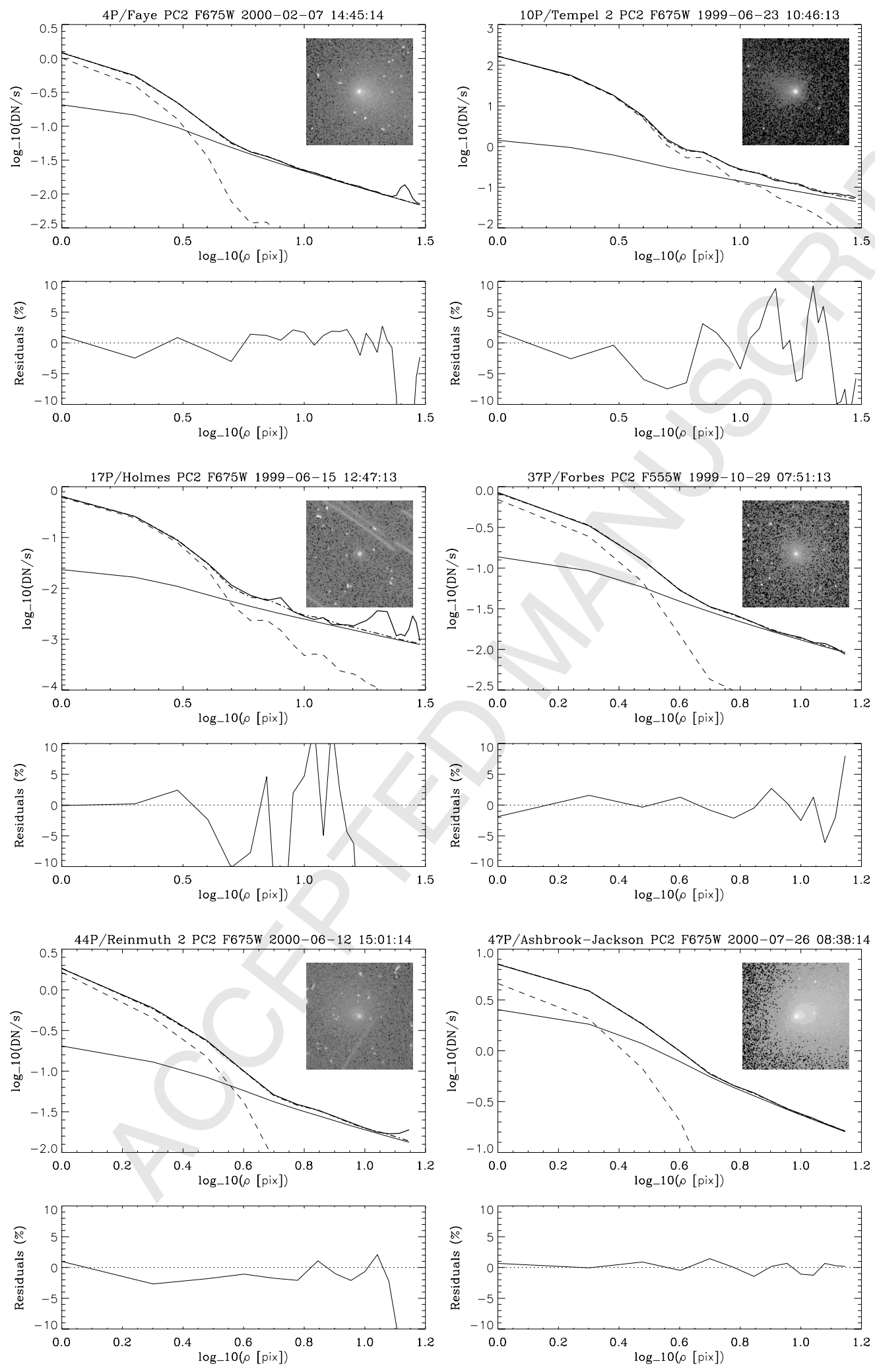

Fig. 1a 

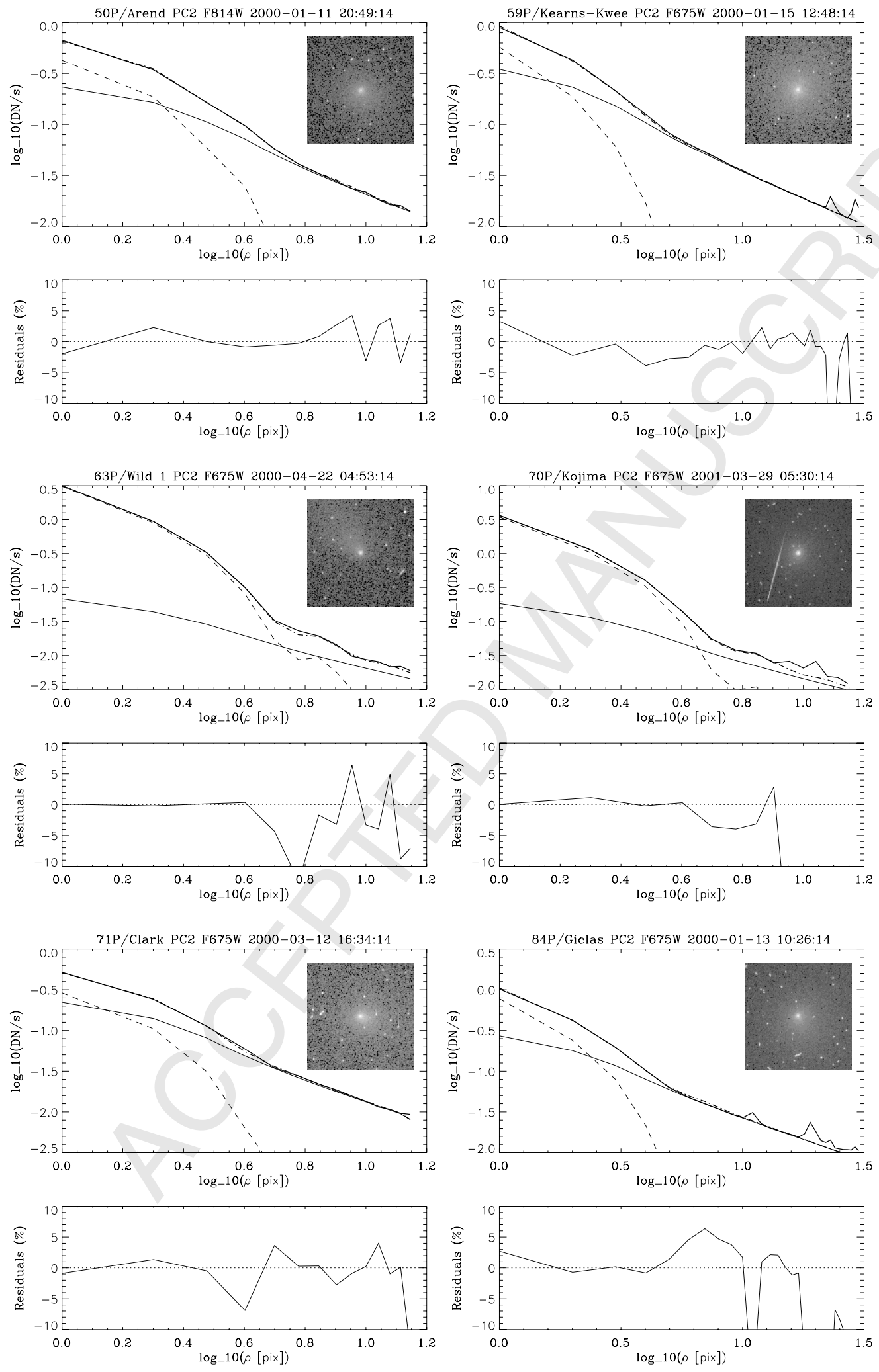

Fig. 1b 

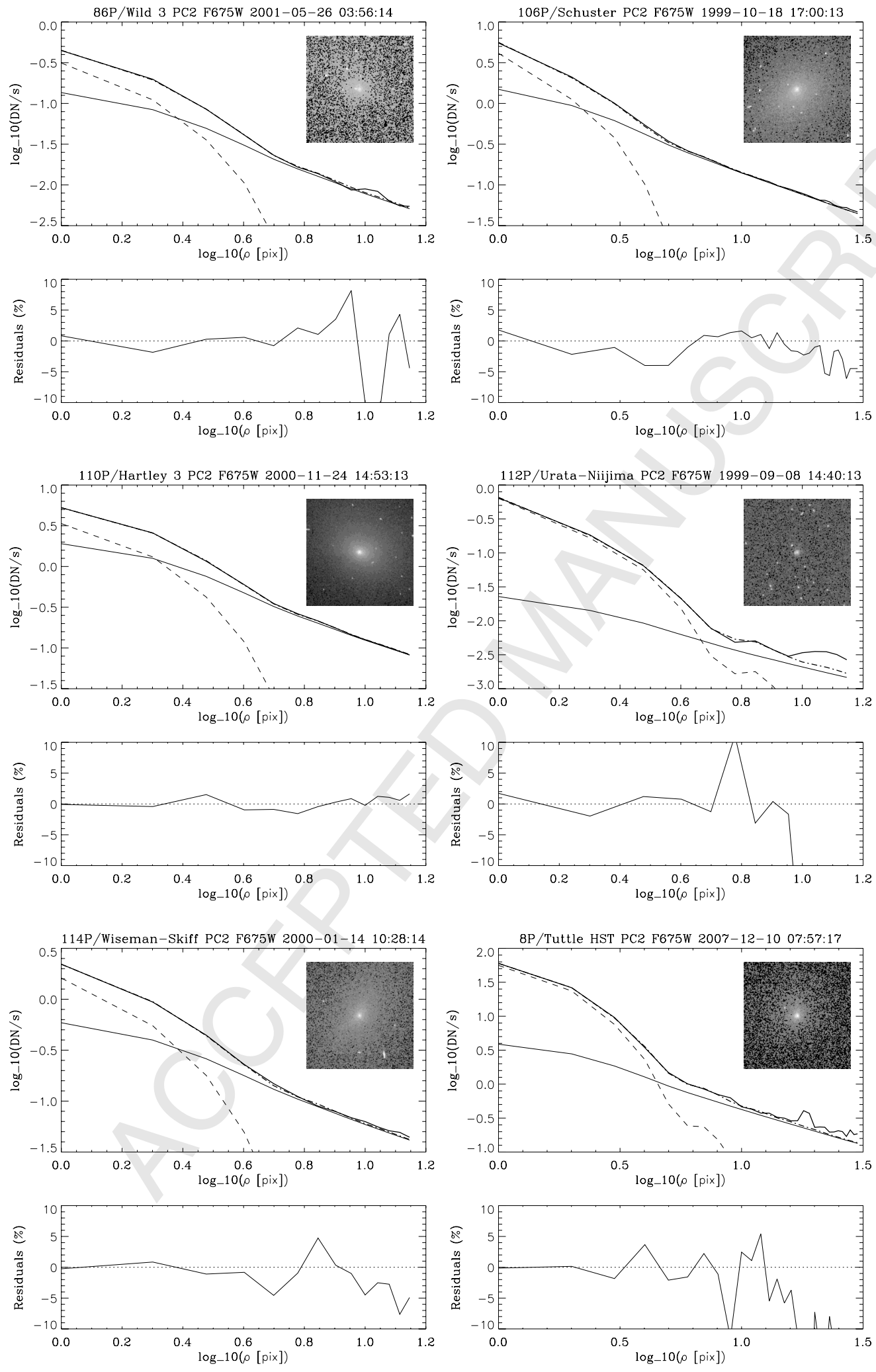

Fig. 1c 


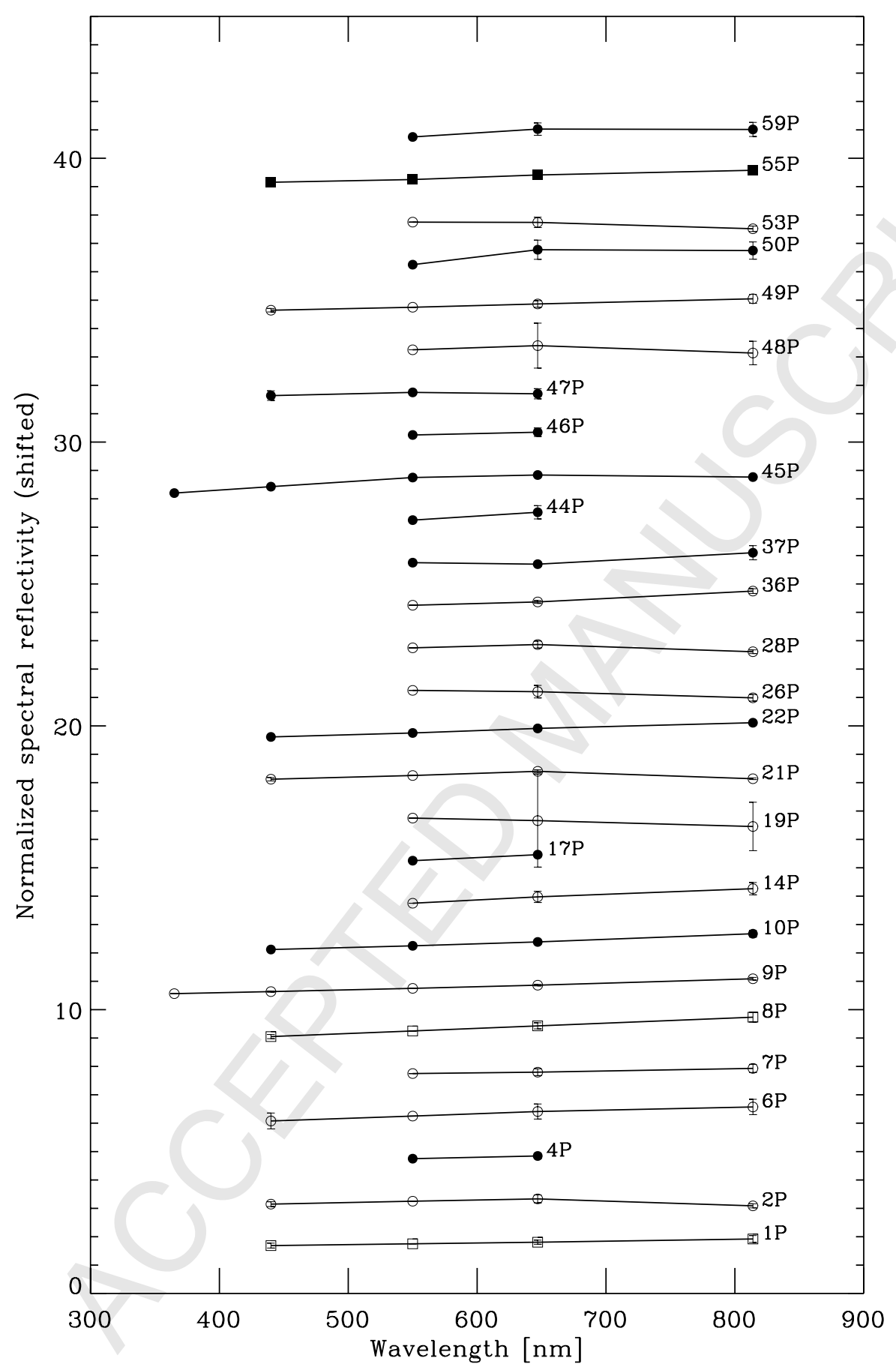

Fig. 2a 


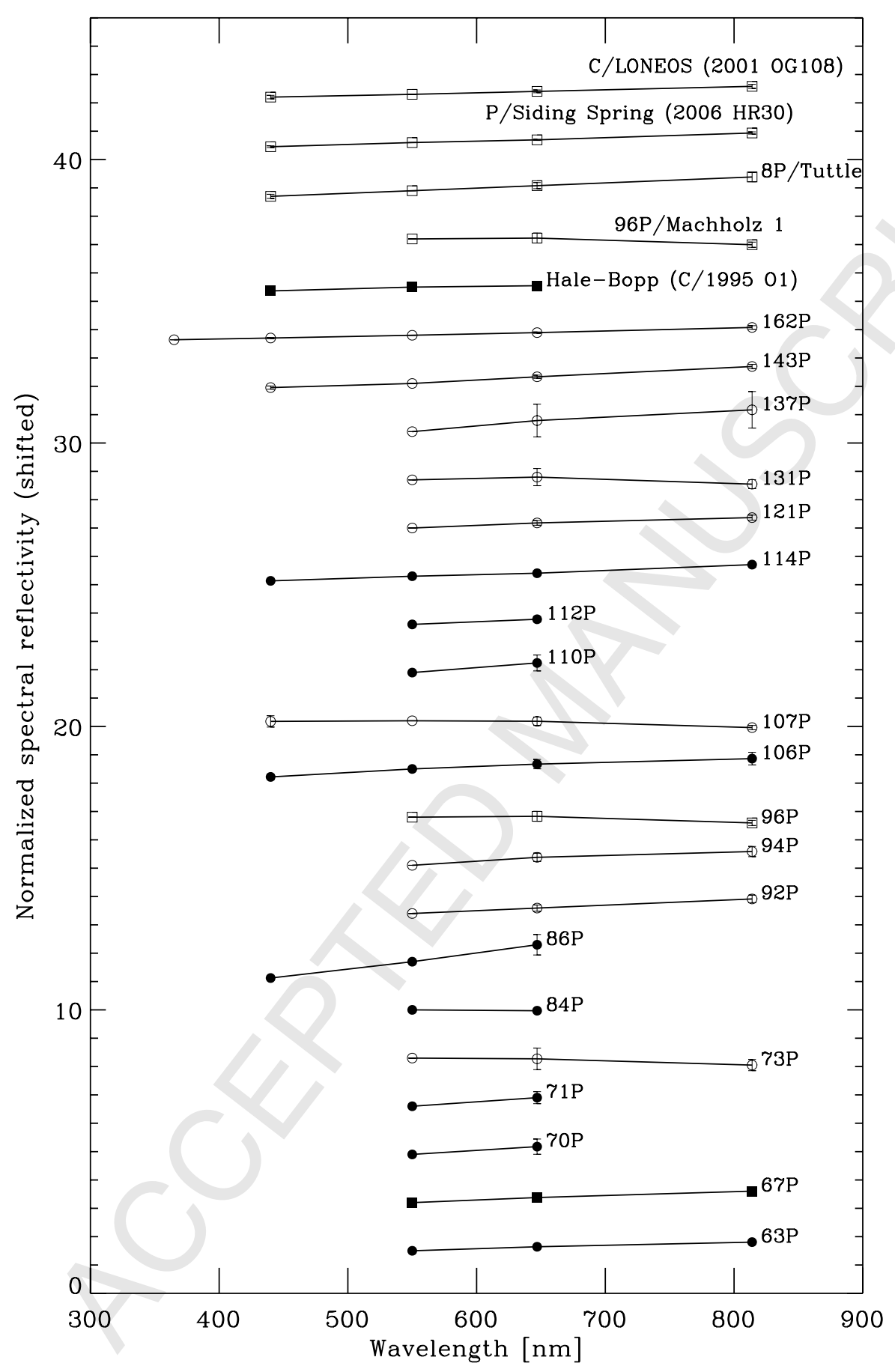

Fig. 2b 

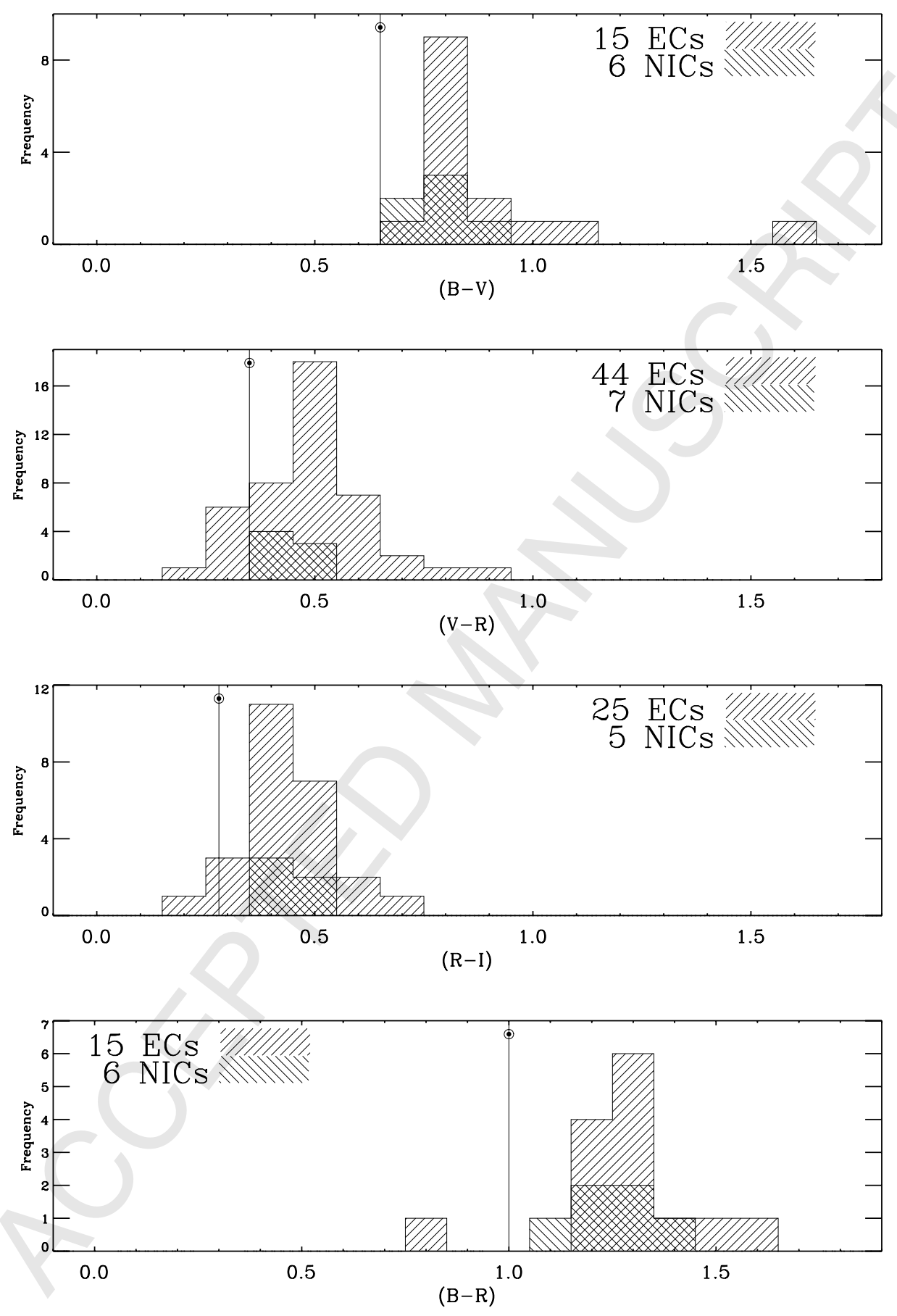

Fig. 3 

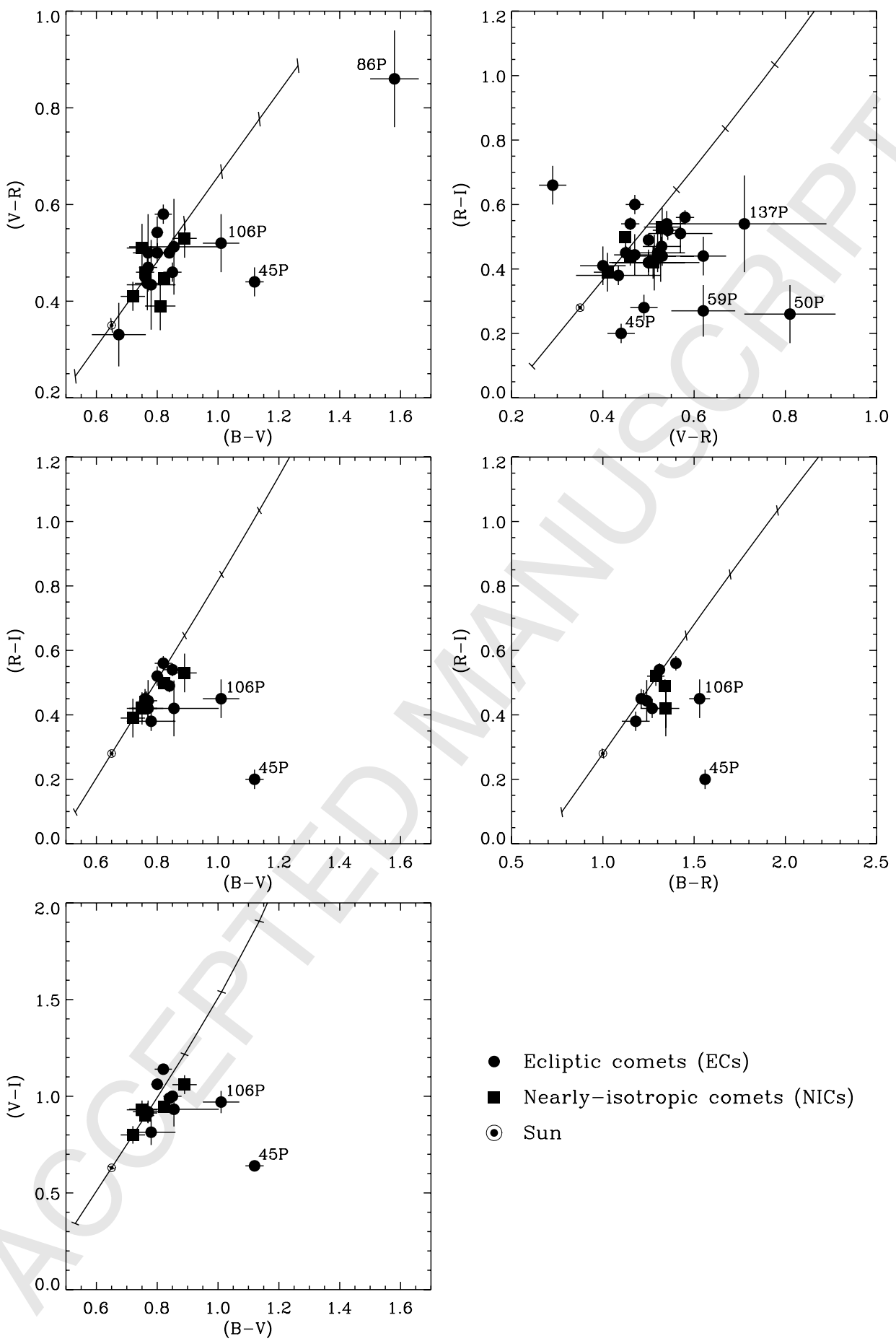

- Ecliptic comets (ECs)

- Nearly-isotropic comets (NICs)

- Sun

Fig. 4 

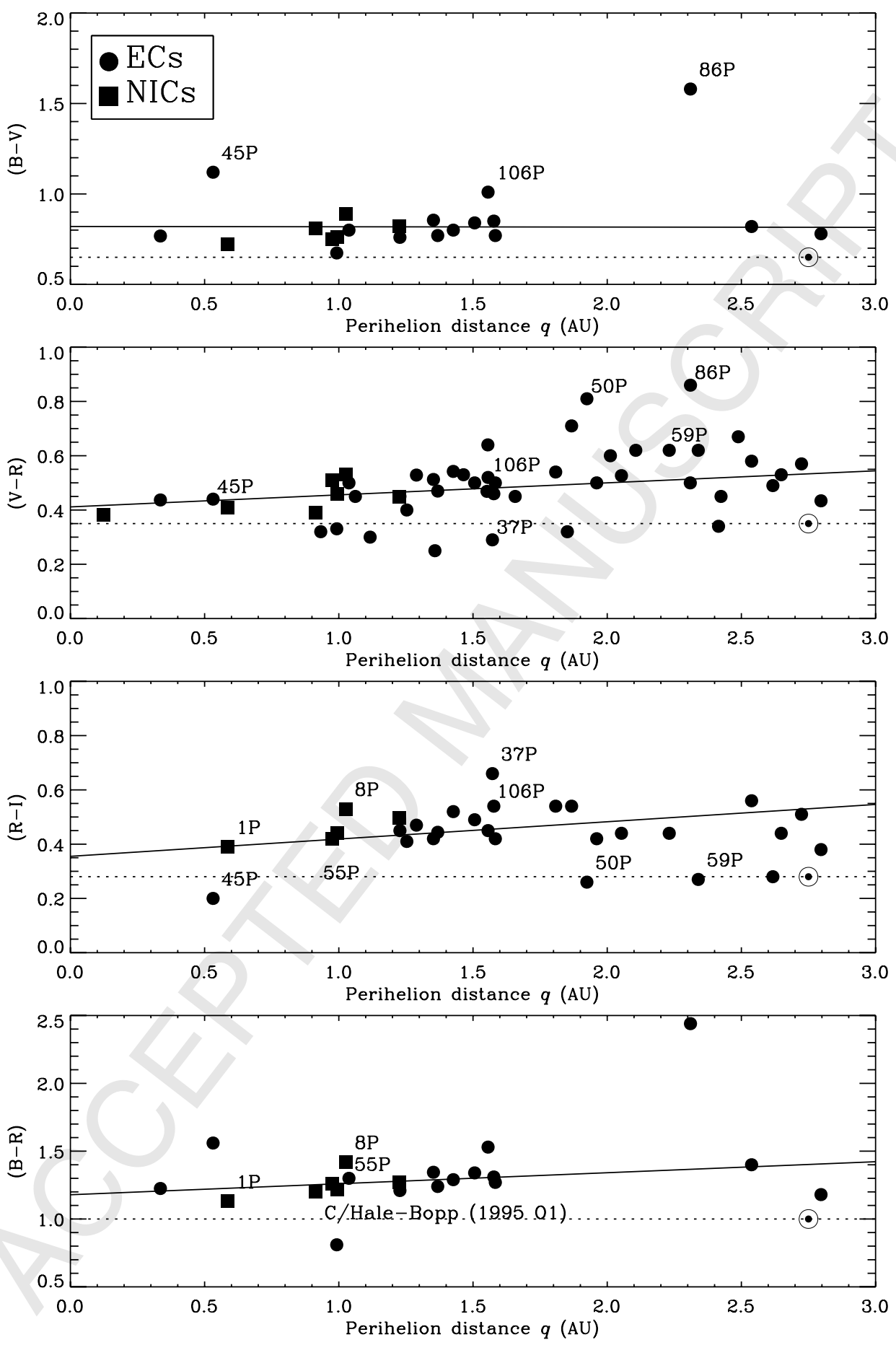

Fig. 5 

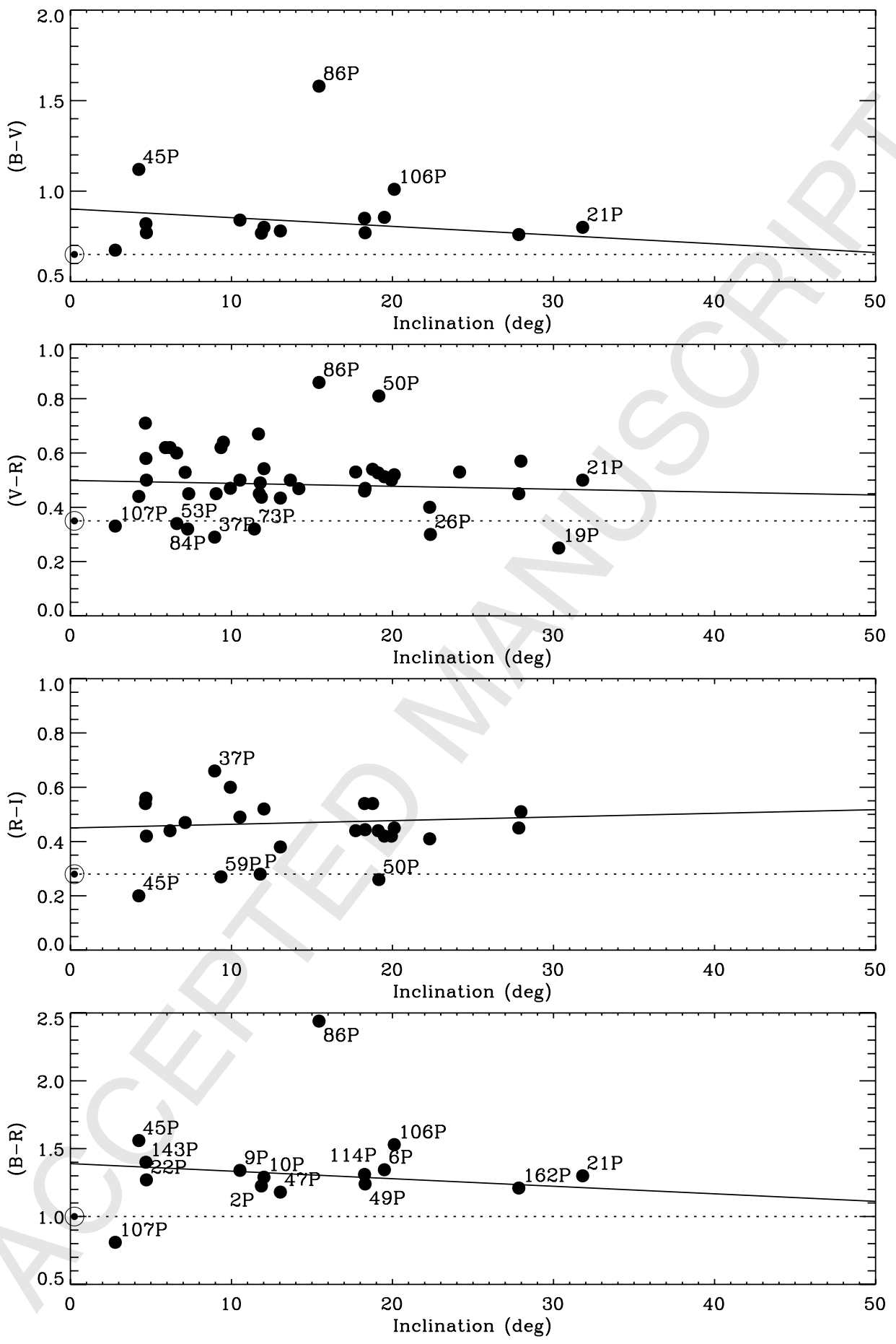

Fig. 6 

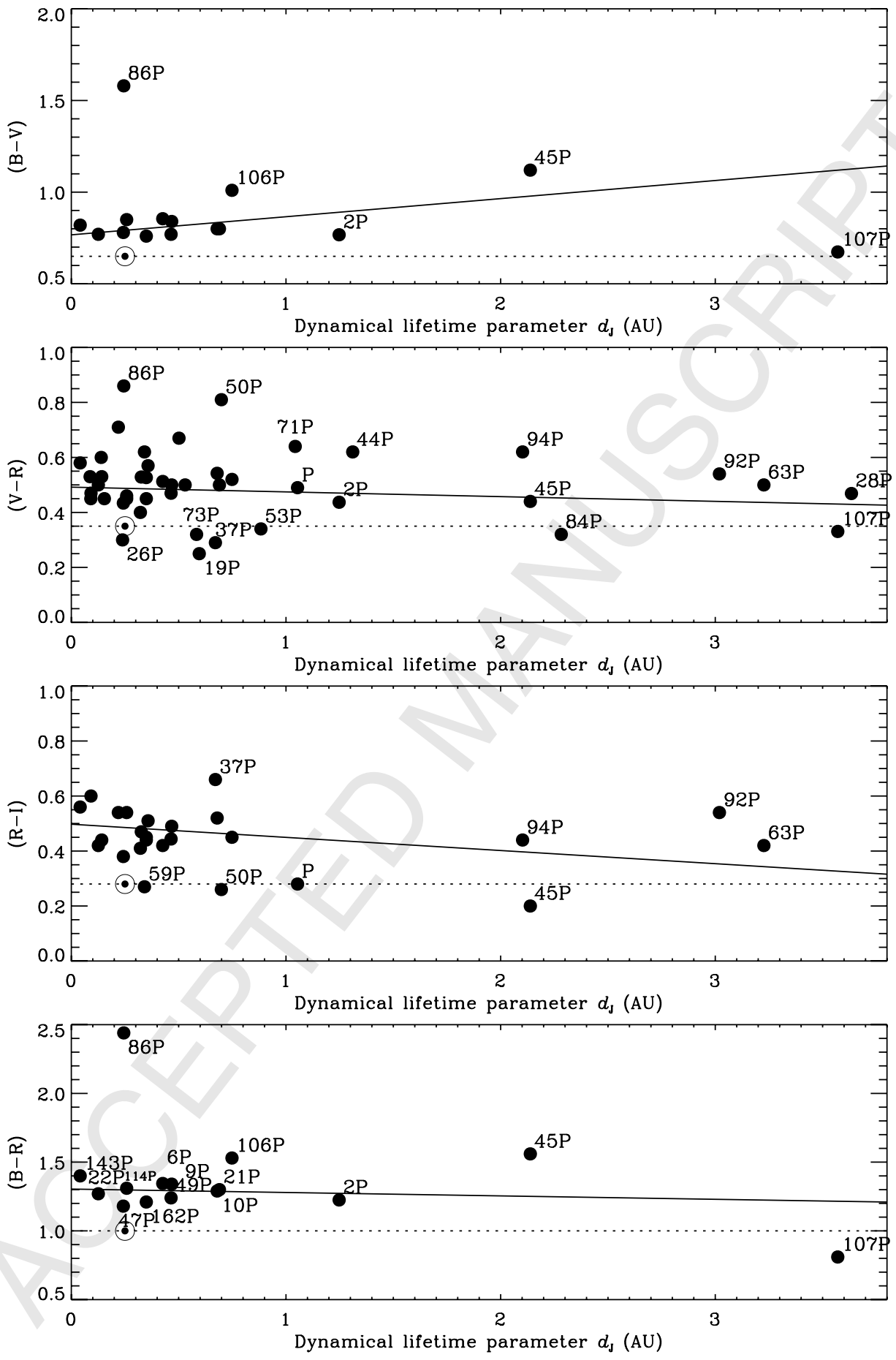

Fig. 7 

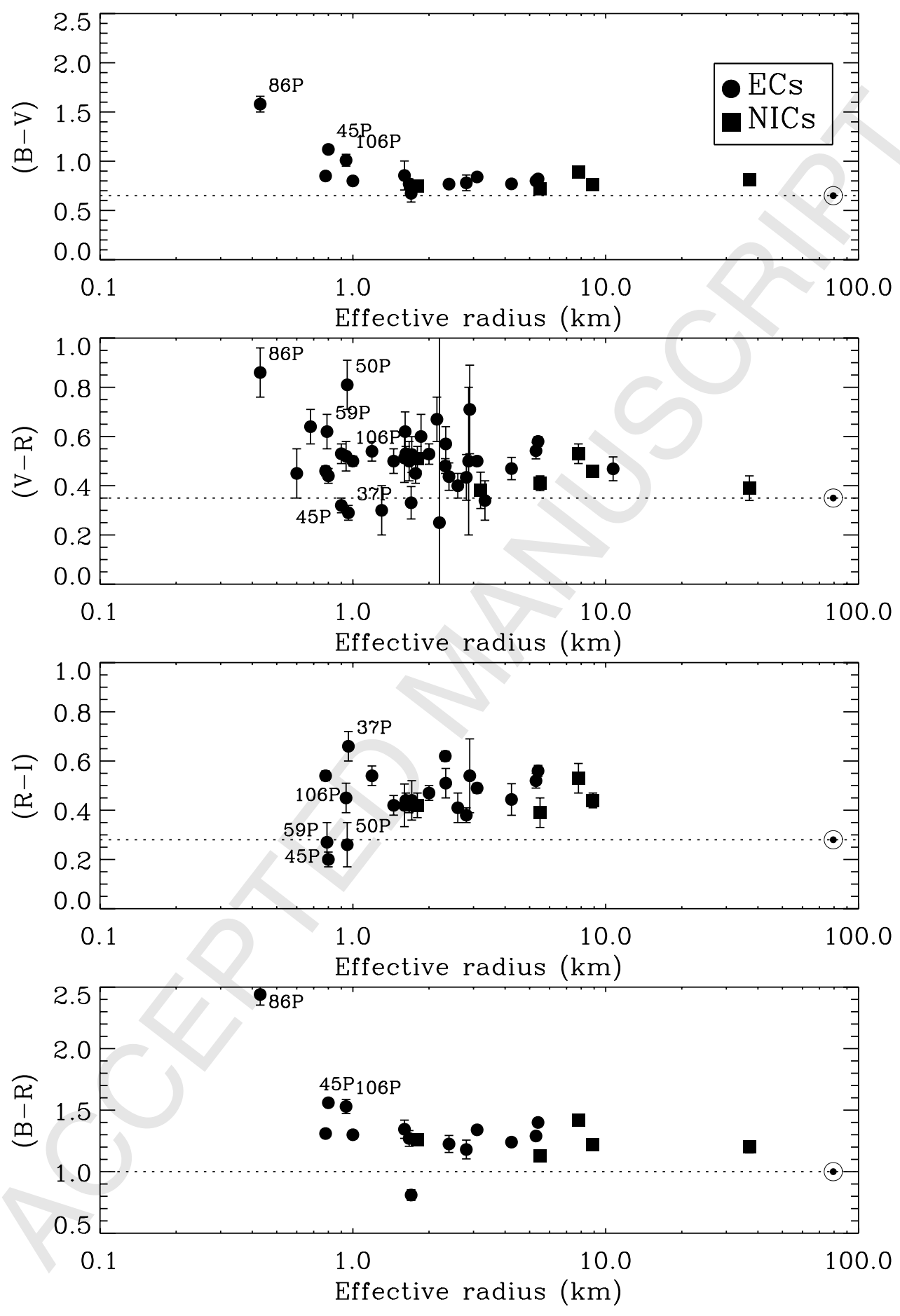

Fig. 8 


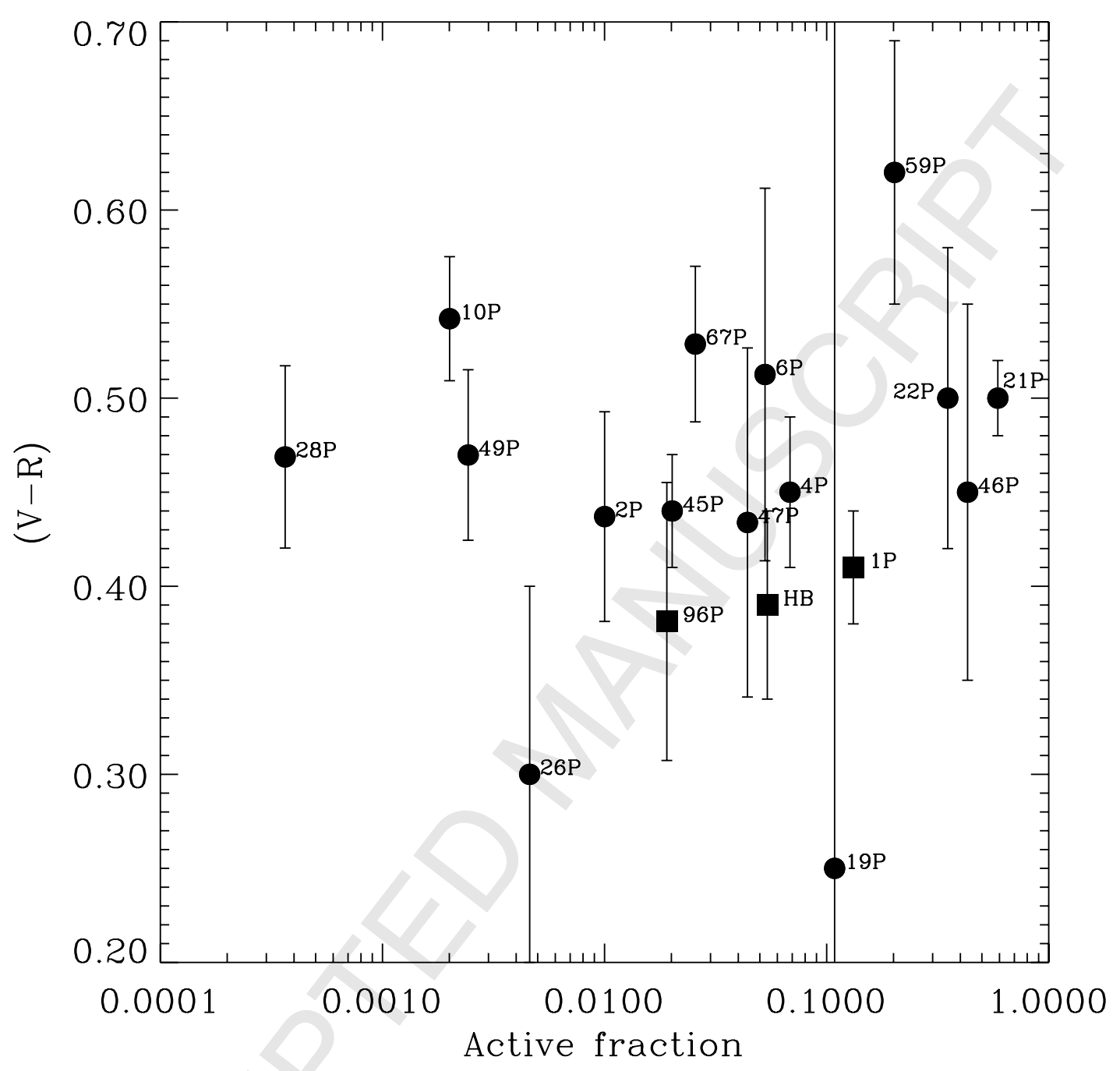

Fig. 9 

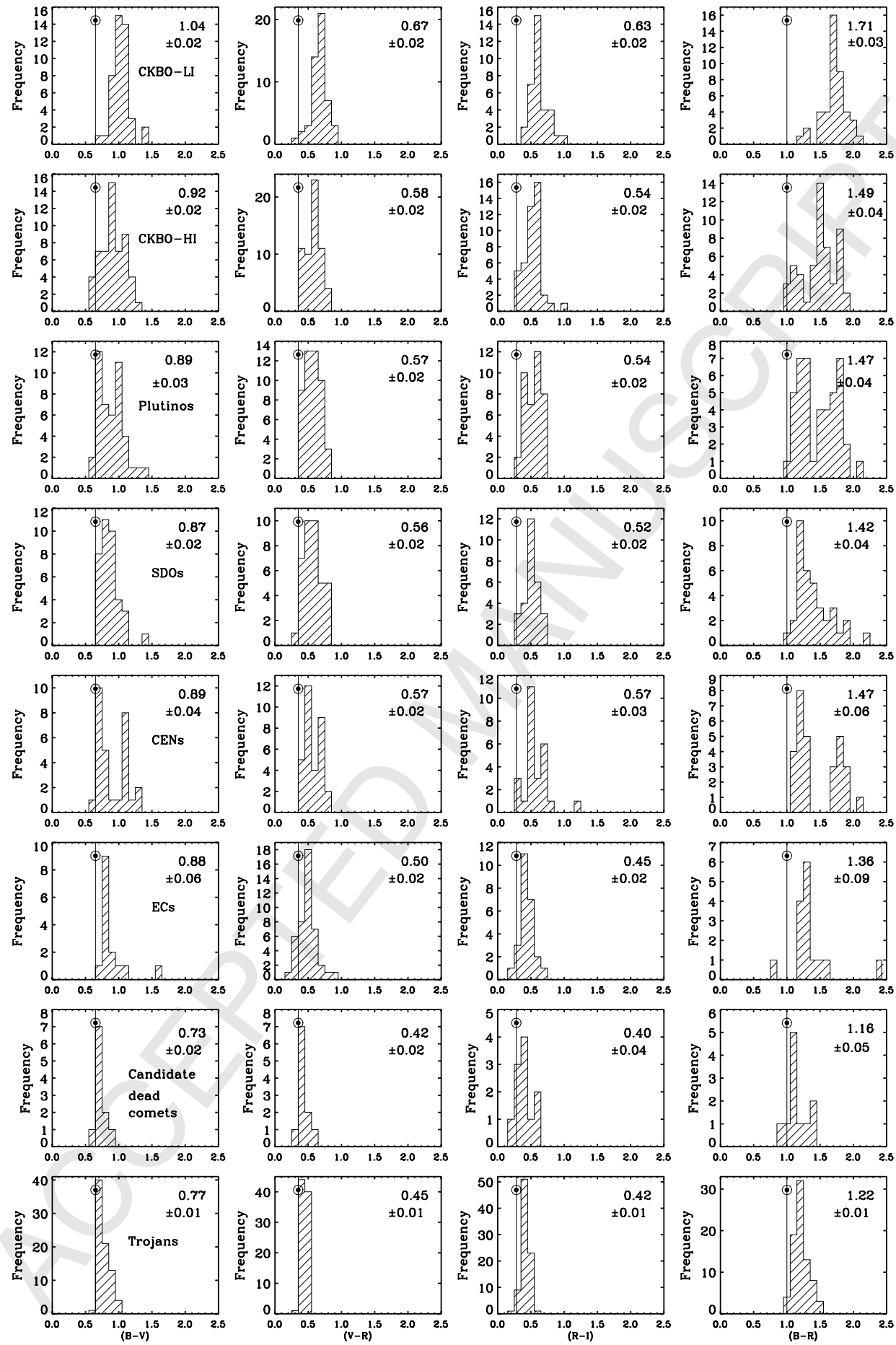

Fig. 10 

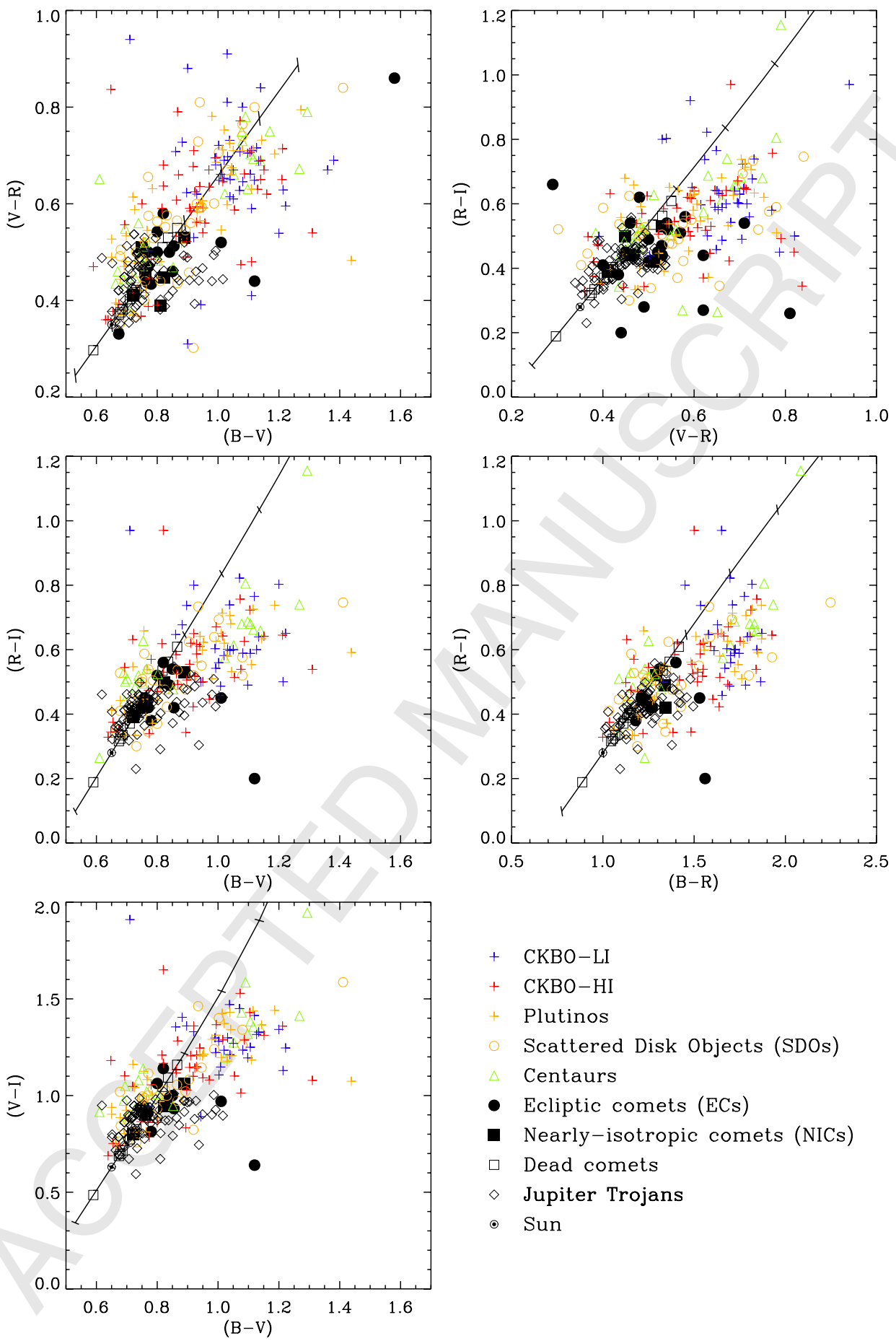

CKBO-LI

CKBO-HI

Plutinos

Scattered Disk Objects (SDOs)

Centaurs

- Ecliptic comets (ECs)

- Nearly-isotropic comets (NICs)

$\square \quad$ Dead comets

$\diamond$ Jupiter Trojans

- Sun

Fig. 11 


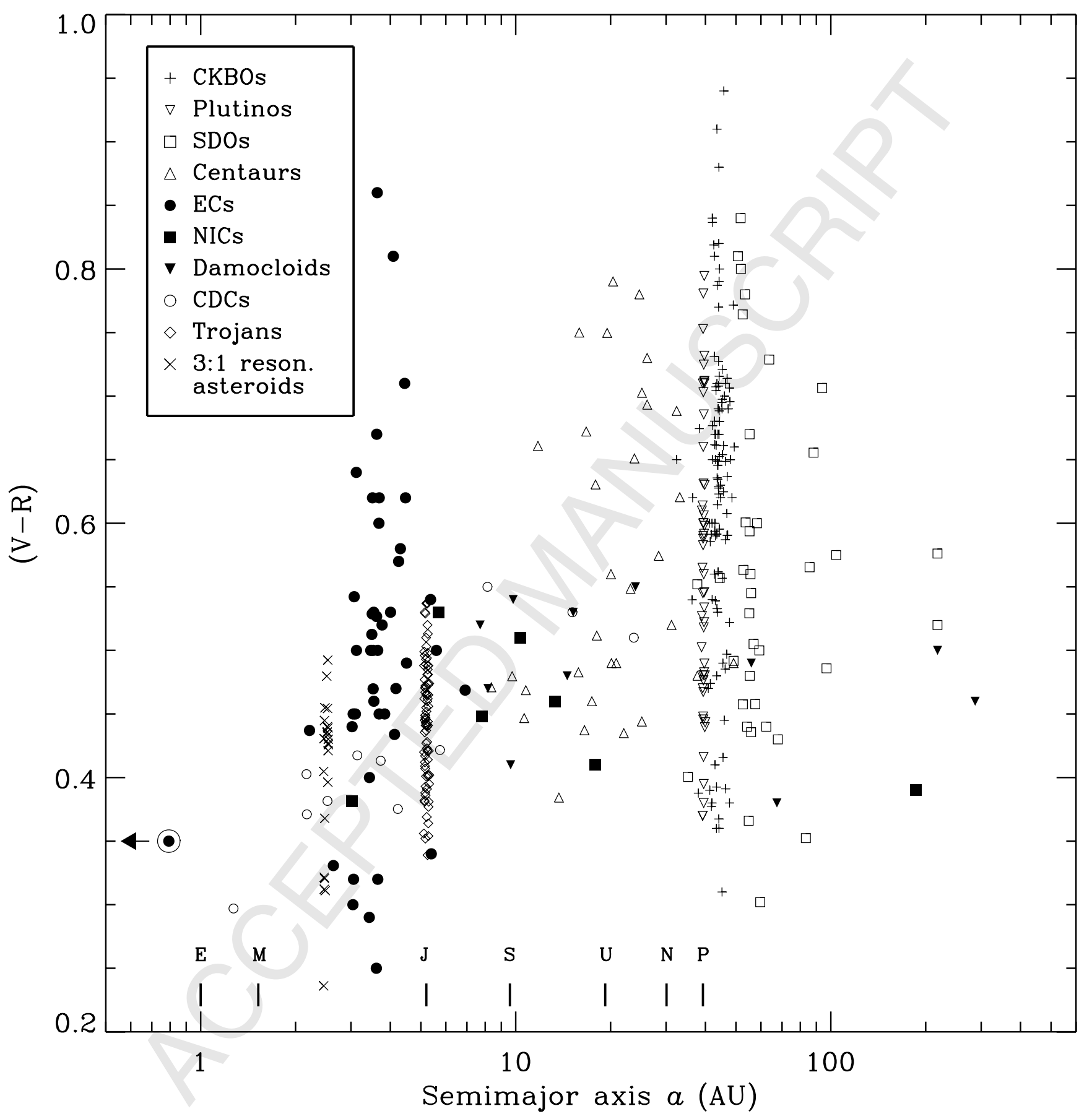

Fig. 12 

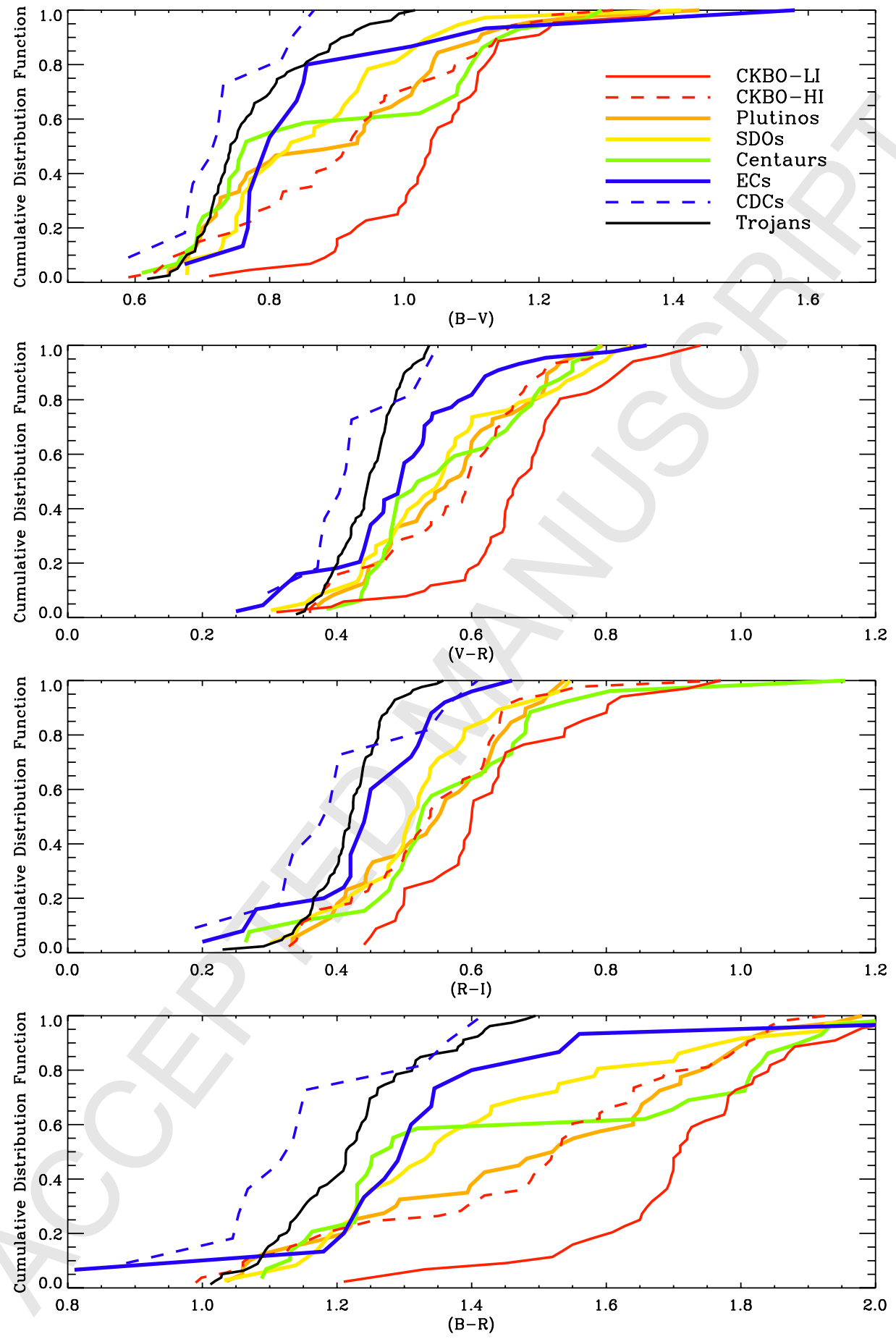

Fig. 13 

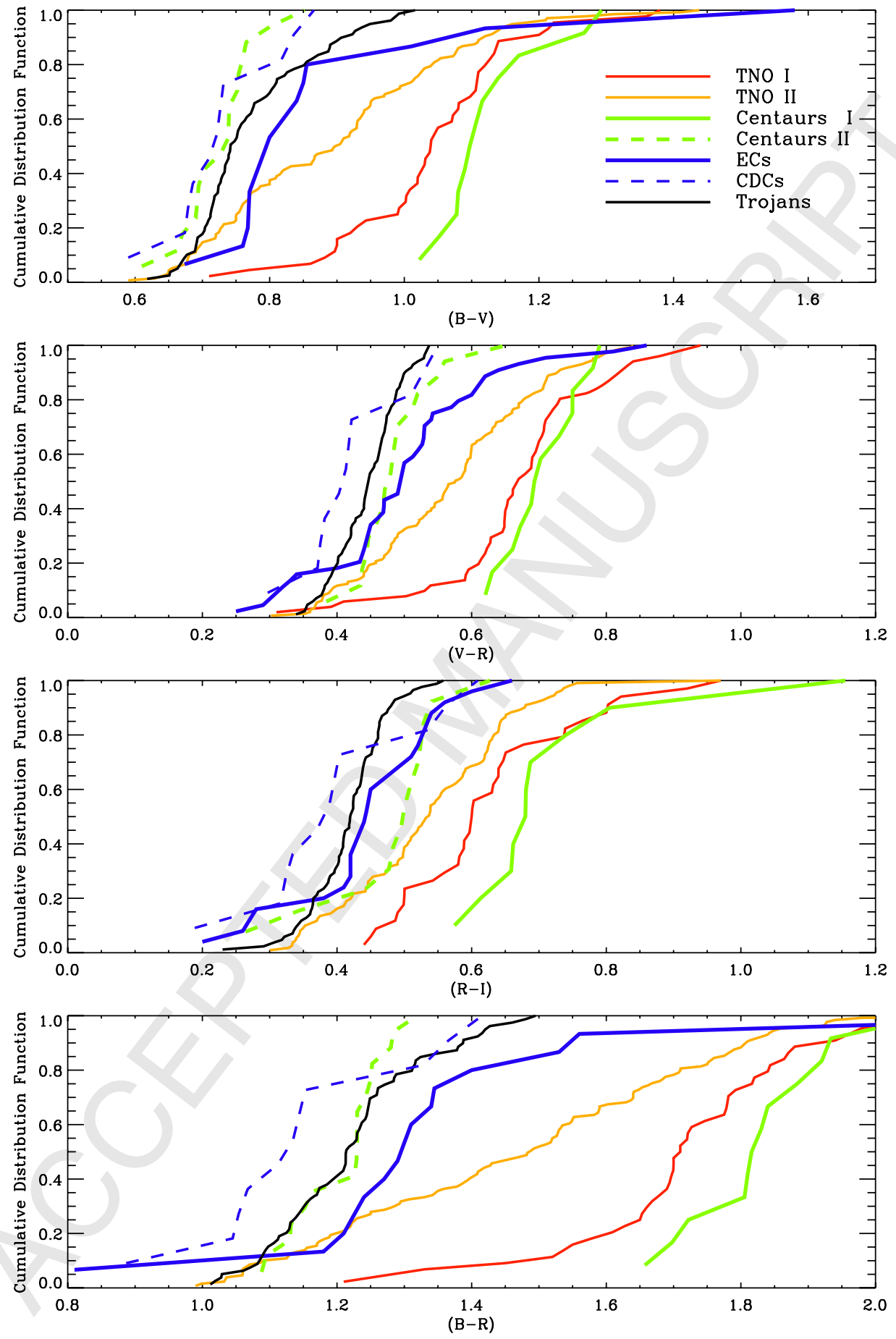

Fig. 14 

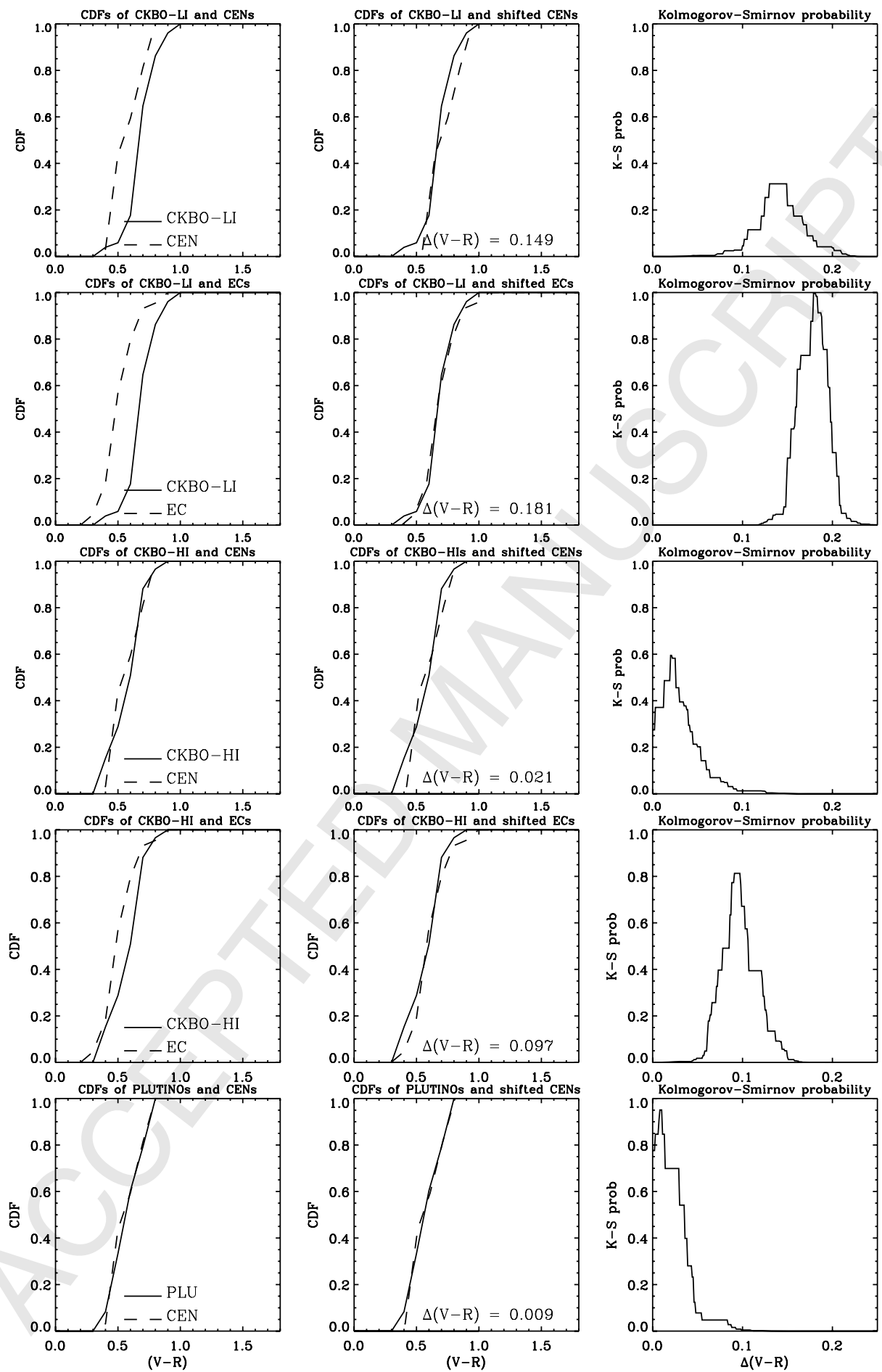

Fig. 15a 

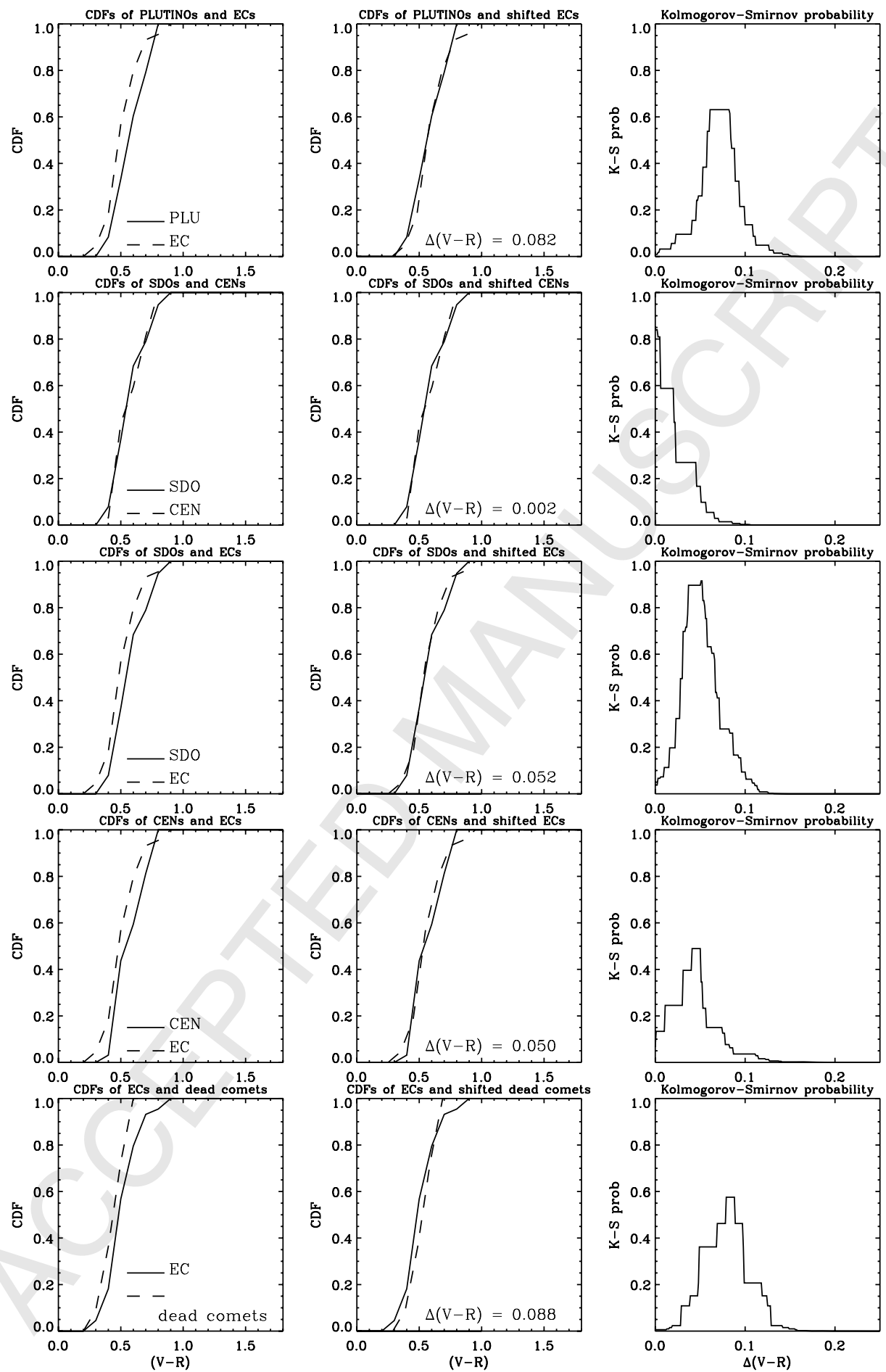

Fig. 15b 

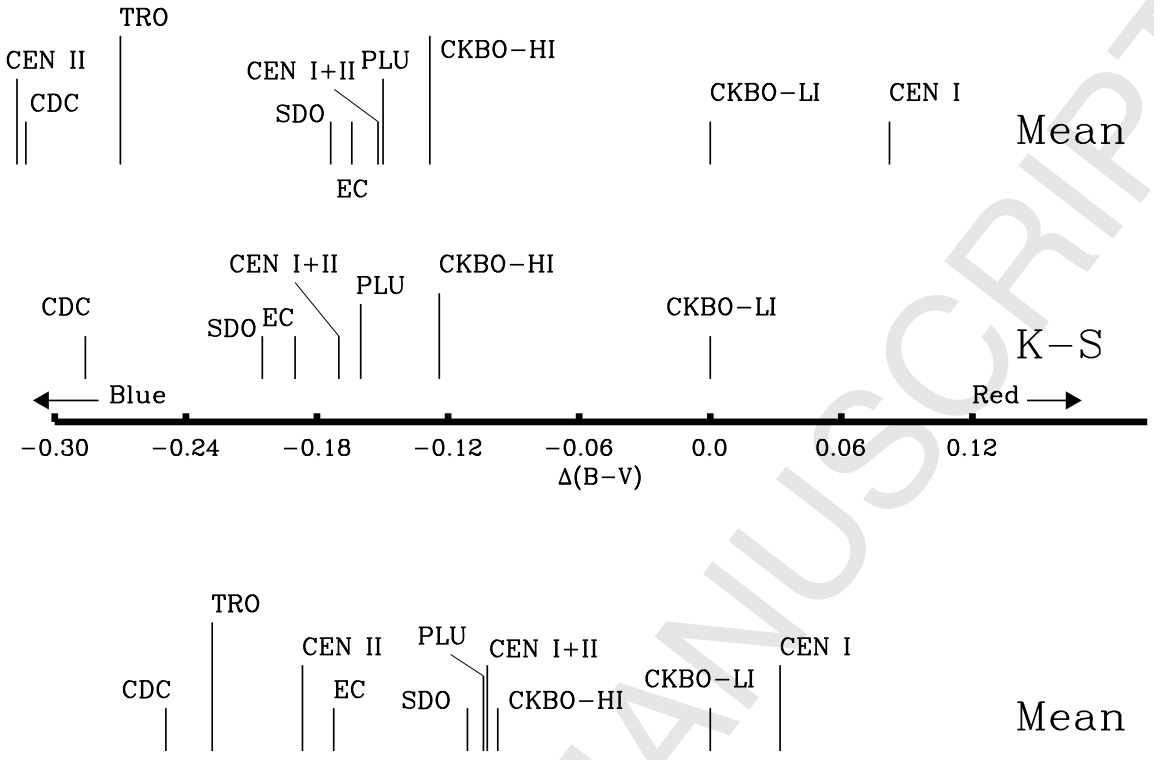

Mean
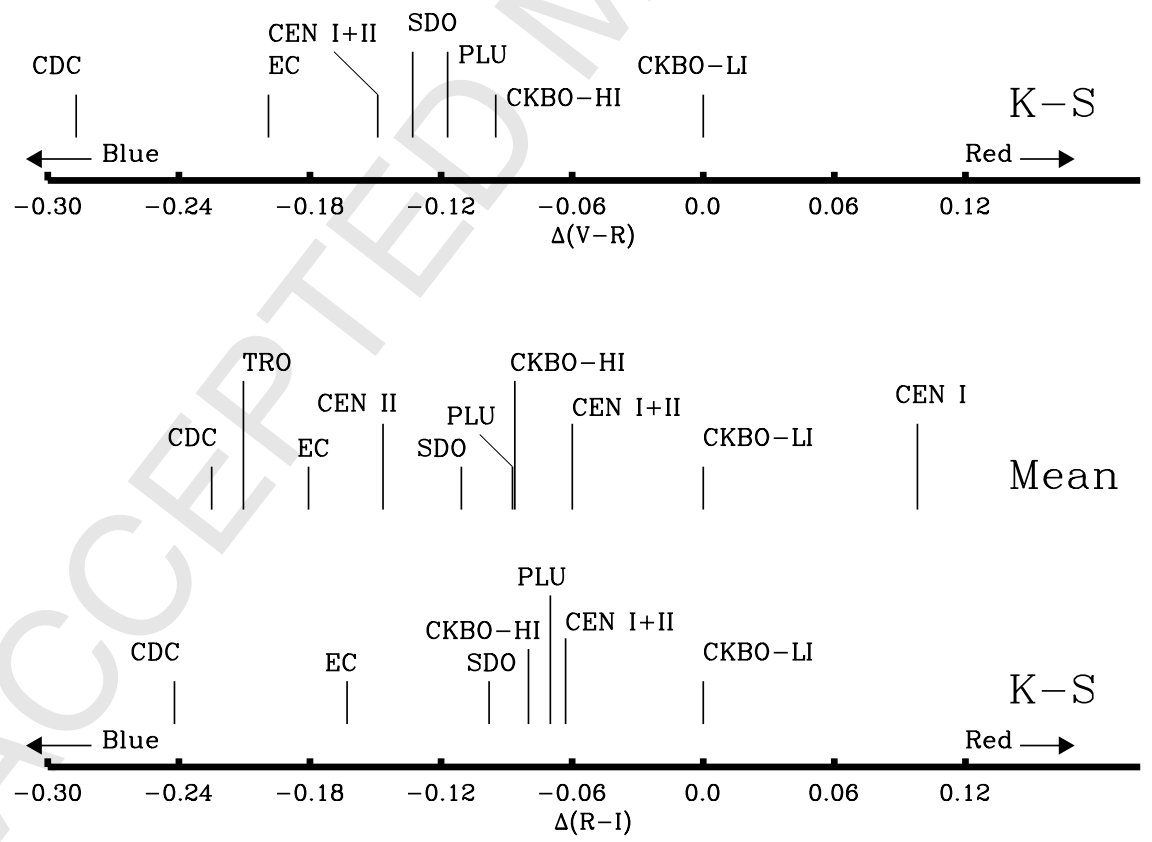

Fig. 16 